\title{
PROCEEDINGS OF THE FORTY-NINTH ANNUAL MEETING OF THE AMERICAN SOCIETY FOR CLINICAL INVESTIGATION HELD IN ATLANTIC CITY, N. J., MAY 6, 1957
}

\author{
Presidential Address
}

\section{THE TRADITION OF SCIENTIFIC CRITIQUE}

\section{By STANLEY E. BRADLEY}

The American Society for Clinical Investigation upholds an old and revered tradition of service to the ideal of a scientific medicine. Within the fabric of our art the threads of learning, skill, and humanity are inseparably woven. When any one of them is lacking or unduly emphasized, the texture is weakened and defective. Unfortunately, throughout most of recorded history, medical science-so called-has been a ridiculous mixture of error, empiricism and superstition. The great physicians, from Hippocrates to Sydenham, held fast to the view that skill and humanity are both best served by an exact and unclouded knowledge of living processes, that medicine is ideally a science to be cultivated by thoughtful investigation. This tradition could not bear fruit, however, until scientific medicine became a realizable possibility with the discovery of the scientific method in the fourteenth and fifteenth centuries (1). Almost immediately civil and ecclesiastical authority proscribed the new way of thinking in the universities (2-4) and for a moment seemed to threaten its development. At this point a new and unexpected element entered the picture.

Scholars and men of affairs, drawn together by a common enthusiasm, formed learned societies to defend and exploit the new method (5). By adherence to the highest possible standards of excellence in work and conduct, by submission to an objective and balanced critique, and by setting an example of intellectual probity, these associations exerted a remarkably pervasive influence in promoting the rise of modern science. The creation of a scientific medicine was defined as a prime objective of the new movement from the very beginning $(1,4,5)$. Medical men figured prominently (indeed proved essential) in the conception, gestation, birth, and growth of science (1) but the profession at large was slow in realizing and profitting by its benefits. Then, almost explosively, in the course of a single century, scientific medicine became an overwhelming reality. Again the schools were reluctant to accept the new ideas, and again scientific societies, having no official academic connection -in this country, the Association of American Physicians and the Young Turks-were needed to promote the revolution in medical teaching and practice. Our Society may take its place in the ranks of the older organizations not only because it maintains the same tradition of a scientific critique but also because its vicissitudes and problems are similar.

The idea of the learned Society had its inception in the activities of a variety of lay organizations-luncheon clubs, fraternities, mystical brotherhoods and the likeall concerned with an exploration of the new ideas generated by the rejection of ancient authority. Out of these emerged the first organized scientific society, the Accademia del Cimento, founded in Florence three hundred years ago in 1657 by the Medici family (5). North Italy had been a "forcing bed" of the method and it is not surprising that during the 10 years of its existence the Florentine Academy was able to attract a remarkable group of men whose publications provided a kind of laboratory manual for the development of early scientific investigation. The English were not far behind the Italians and they built more lastingly. In fact, the Italian organization was patterned upon a Utopian research institute sketched out by an Englishman-the "House of Solomon" in Francis Bacon's New Atlantis. Bacon's program also formed the basis upon which the Royal Society of London was incorporated in 1660 (6). Academies of science sprang up in France and Germany under the same influence.

The Royal Society survives today as a vital and vigorous institution despite a long history of typical upsand-downs (6). Approximately a third of the founding members were professionally trained and intellectually disciplined scholars; the remainder were political, military and even ecclesiastical notables with an avocational attitude rather than a primary dedication to the new science. This division persisted for years-at first beneficially, later with detrimental effect. The prestige resulting from the patronage of the Crown, the nobility and men of wealth did much initially to enlist popular sympathy and support. The amateurs provided an enthusiastic and intelligent audience but unfortunately they brought ridicule and derision upon the Society. Their extravagant speculations and absurd preoccupations gave substance to the scoffers' claim that learning produced a gullible crack-pot interested only in the exotic. Strong leadership and the work of the more critical members tended to counteract this picture during the early eighteenth century but by the end of the century the Society had again fallen into disrepute. The non-professional element had tended more and more to regard the organization as a social club, nomination was tantamount to election, and scientific ability did not weigh as heavily as social position and religious views in determining membership. To make matters worse, new, apparently competitive, scientific organizations began to appear; on the one hand, devoted to the cultivation of special fields such as botany and geology and, on the other, concerned 
with the general political and social interests of all scientists, as typified by the British Association for the Advancement of Science. With the growing complexity of mature scientific disciplines, a professional attitude of responsibility, stability, and dedication to a stern critique was needed for continued progress. Reorganization of the Royal Society in the 1840's with the establishment of a membership policy designed to limit the membership and to assure suitable qualifications brought the professional attitude to the fore in its affairs. As a result of insistence upon a high standard of excellence in its membership, publications and other activities, the Society quickly regained a position of leadership and fills today a unique position occupied neither by the special societies nor by the British Association, a position in which it embodies and exemplifies the best in scientific critique.

The parallels between the story of the Royal Society and our own need not be labored. We, too, are faced with the problems posed by an unsettled membership policy, by an ill-defined relationship to other groups, and by pressure for expansion. Throughout its long history the Royal Society exerted a remarkable influence primarily because it set the highest possible standard of excellence. With a falling away from the standard its influence waned, to be re-established only with restoration of the standard at a high level. Our Society also has stood for quality, for the highest possible standards of excellence in membership, in the Journal and in the papers presented before this meeting. We can celebrate the tercentenary of the foundation of the learned Societies in no more fitting way than by rededicating ourselves to that ideal.

To the best of my knowledge no conscious plan was ever laid down to attain our Constitutional objectives, but the size and interest of the audiences we are privileged to entertain each year suggest that the Society may have been successful in spite of itself. Medical education has undergone an extensive revision in the years since the first meeting and there is good reason to believe that many members played a major role in the process. In time the triumph of the ideals to which those men subscribed gave lustre to the Society. The standards they established in selecting members reflected great credit upon and gave distinction to the fact of membership itself. The Journal benefitted as a result of the conspicuous quality of the papers it was able to attract. The conscious emulation of the accomplishments of the founding members has done much to promote "the cultivation of clinical research by the methods of the natural sciences" throughout this country and to encourage "the diffusion of a scientific spirit."

Of even greater importance has been the change in the practice of medicine during these years. There is little doubt that now science and practice are closely blended, a result born of dire and urgent necessity. In no other group of professional workers is the scientific attitude more vital or more meaningfully a matter of life and death. Practice today is truly clinical investigation, demanding at once a vigilant critique, a full understanding of the human situation, and a knowledge of fundamental scientific tech- niques. As it strives to assist in maintaining a high standard of excellence in clinical research, the Society fully serves the ends for which it was founded.

No standard that lacks a divine sanction is readily acceptable; resentments, jealousies, fears, a host of varied considerations combine to raise up an active opposition to it. In each generation the intellectual standards, which professional men live by, must be tested and reaffirmed. This fact was abundantly confirmed in the history of the Royal Society and we should not be surprised that we also must face up to what we are apt to call the "antiintellectualism of our time," as if it were a problem uniquely ours. Today we are confronted by a widespread assault upon science in medicine. Lip service is paid to its rewards, but an astonishing effort is expended in traducing it. The "scientific spirit," for which this Society stands, is said to produce a cold disinterested character, to dry up the milk of human kindness, whereas a training in social and emotional welfare at the expense of scientific discipline instills by some magic all the human instincts. This is essentially an attack upon standards, an attempt to condone amateurism by redefining it, an effort to make the easy way the right way. I, for one, cannot accept the implication that thought and feeling are incompatible, that the emotional response can somehow supersede, and even take precedence, over an intellectual evaluation. As long as we accept the principles upon which this Society was founded, I think we are all committed to a balanced criterion in which truth and spirit are interlocked; an unattainable ideal perhaps but one vital to progress. The tendency to dissociate feeling and intellect has disastrous effects upon judgment. It provides an excuse to dispense with critical integrity; to measure our friends and our own interests against a different standard than the one we use for outsiders, or to take the easy road of approving of everything and every one in a wonderful world. Unhappily, an unfavorable decision is wounding and difficult of tactful explanation, obviously subject to error, often creating the false impression of intellectual arrogance.

Criticism of the Society is especially acrid in connection with the choice of new members. In eight years of work on the Council of this Society I can say with pride that I have never seen any consideration other than merit take first place in determining nomination though geographical discrimination is often urged upon us. At present the membership is distributed through the land in approximately the same proportion as the population at large. The Western states are somewhat favored in the selection of our officers since every President appoints a nominating committee of three, one each from the Eastern, Central, and Western states and the three nominees selected are usually distributed in the same manner. In this way some 14 per cent of our membership can control one-third of the official positions. This would seem to be a wholly justifiable method of giving encouragement to the West but merit alone should determine our selection of candidates for membership. More recently there has been pressure to increase the membership on the assumption that the number of new 
members should be determined by the number of men nominated. The history of the Royal Society shows the fallacy and danger of this assumption. If we wish to allow for the growth of the research establishment in this country, we might gear membership to the number of workers listed in American Men of Science, or better yet, to the number of subscribers or contributors to the Journal of Clinical Investigation. Certainly the number nominated is a poor criterion of anything but the prestige of the Society.

We come now to the scientific session. The papers selected for presentation here have been picked from more than 200 submitted. An honest effort has been made to take the best from a group of excellent titles without reference to subject, geography, or personalities. This is not an easy task; it is certainly never discharged to everyone's satisfaction. Nor are these easy papers. Medicine is a hard and demanding mistress today, always on the move, always changing her focus and concentration. What we learn today will tomorrow seem commonplace, foolish, or obsolete, but now it is new and difficult. As long as progress is being made this must be so; there is no advantage in not facing the fact and swallowing the bitter broth of novel and complex concepts. We must seize upon our facts where we find them, whether by the bedside, at the laboratory bench, or in the animal quarters. In its broadest possible sense clinical investigation is as deeply concerned with the metabolism of the viral particle or animal cell as it is with the behavior of the whole man. Every fact, concept, or method that may have some ultimate bearing upon the problems that face us as physicians should have a hearing in this Hall.

We perpetuate a long and proud tradition that goes back at least 300 years, an intellectual tradition of undaunted critique and of adherence to a high standard of excellence. In these yearly rites of Spring we pay tribute to that tradition and to the view that medicine is a scholarly profession steeped in mercy and dependent upon a devoted skill. To be effective, head, heart, and hand must work as one.

\section{REFERENCES}

1. Randall, J. H., Jr., The development of scientific method in the school of Padua. J. Hist. Ideas, 1940, 1, 176.

2. Chaplin, A., The history of medical education in the Universities of Oxford and Cambridge, 1500-1850. Proc. Roy. Soc. Med. (Sect. Hist. Med.), 1920, 13, 83

3. Cawadias, A. P., Thomas Linacre and the first scholarphysicians of Oxford. Brit. Med. J., 1936, 2, 550.

4. Allen, P., Medical education in 17th Century England. J. Hist. Med., 1946, 1, 115.

5. Ornstein, M., The Rôle of Scientific Societies in the Seventeenth Century. Chicago, University of Chicago Press, 1928, 308 pp.

6. Stimson, D., Scientists and Amateurs. New York, Henry Schuman, 1948, 270 pp. 


\section{ABSTRACTS}

Cardiorespiratory Effects of Epinephrine Inhalation in Normal Subjects and Patients with Chronic Pulmonary Emphysema. James K. Alexander, Junichi Mise, Edward W. Dennis, and Robert L. Hershberger, Houston, Tex. (Introduced by James A. Greene).

Observations were made in 5 normal subjects and 12 patients with chronic pulmonary emphysema. Both groups showed increases in pulmonary ventilation, tidal volume, and oxygen uptake, with no significant changes in respiratory frequency, physiological dead space to carbon dioxide or respiratory change ratio. No significant change in arterial blood oxygen saturation or $\mathrm{pH}$ occurred, but arterial $\mathrm{CO}_{2}$ tension fell in the emphysematous patients, suggesting a greater efficiency of alveolar ventilation in this group following the drug.

There was an increase in heart rate and cardiac output in both groups, with reduction in the arterio-venous oxygen difference. Stroke volume and systemic arterial pulse pressure increased or remained the same with no change in mean pressure. Pulmonary arterial mean pressure rose in all the normal subjects and the majority of patients with emphysema, associated with an increase in pulmonary arterial pulse pressure. Calculated vascular resistance in the systemic circulation fell. Pulmonary vascular resistance was unchanged in the normal subjects and the majority of the emphysematous patients, but fell significantly in 3 patients with severe disease. The fall in pulmonary vascular resistance in these 3 patients was not correlated with change in intrathoracic pressure variation, arterial blood oxygen saturation or $\mathrm{pH}$.

Observed increases in pulmonary arterial wedge pressure and right ventricular diastolic pressure, together with a shortened duration of right ventricular mechanical systole, were interpreted as indicative of a change in the dynamics of ventricular contraction. There was a rough correlation between pulse pressure increase and stroke volume change in the left ventricle following epinephrine inhalation in the emphysematous subjects, but not in the normal group.

Abnormalities of Carbohydrate Metabolism of Erythrocytes from Patients with Paroxysmal Nocturnal Hemoglobinuria. K. I. Altman, H. Tabechian, L. E. Young,* M. J. Izzo, M. Murphy, and G. Roberts, Rochester, N. Y.

The possibility of a metabolic parameter in hemolysis of red blood cells ( $r b c$ ) from two patients with paroxysmal nocturnal hemoglobinuria (PNH) was investigated a) by measurements of spontaneous in vitro autohemolysis and b) by studies involving $P^{\text {as }}$ labeling of phosphorylated intermediates of carbohydrate metabolism in the rbc.

Addition of glucose $(30 \mathrm{mM})$ regularly caused slight acceleration of hemolysis of defibrinated $\mathrm{PNH}$ blood ( $\mathrm{pH}$ adjusted to 6.9) during incubation at $37 \mathrm{C}$ for pe- riods of one to five hours unless glycolysis was inhibited, as by sodium iodoacetate $(25 \mathrm{mM})$. Inosine caused marked deceleration of hemolysis. In none of these experiments could the effect of the additives be attributed to change in $\mathrm{pH}$ of the blood or to an anticomplementary effect, but an effect on other serum components of the "PNH hemolytic system" could not be entirely excluded.

When rbc from $\mathrm{PNH}$ patients were incubated with $\mathrm{NaH}_{2} \mathrm{P}^{32} \mathrm{O}_{4}$ there was a relatively slow rate of incorporation of $\mathrm{P}^{22}$ into 2,3-diphosphoglycerate (2,3-DPG) but the total concentration of 2,3-DPG was greater than normal. The rate of entry of extracellular orthophosphate- $\mathrm{P}^{2 a}$ into rbc was considerably slower in $\mathrm{PNH}$ than in normal rbc but was regularly accelerated by addition of $\mathrm{NaF}(5 \mathrm{mM})$ to PNH blood. When PNH erythrocytes were prelabeled with orthophosphate- $P^{2 s}$, the rate of release of $\mathrm{P}^{32}$ into the plasma during subsequent incubation was much slower than the release from normal rbc similarly treated. The rate of release of $P^{a s}$ from $\mathrm{PNH}$ cells was further reduced by addition of $\mathrm{NaF}$ (5 mM).

These findings which in the aggregate are unlike those encountered in any other type of red cell indicate that there are abnormalities of intermediary carbohydrate metabolism in $\mathrm{PNH}$ erythrocytes. The role of these abnormalities in producing hemolysis in vivo is not yet clear.

Iso-osmotic Level of Freezing Point Depression in Boiled Tissues. Johannes W. Appelboom and William A. BRODSKY,* Louisville, Ky.

Since the hypertonicity previously reported in frozen tissues could have been due to rapid metabolism of the homogenates, or to physical changes of proteins subjected to liquid $\mathrm{N}_{2}$ and high pressure, present experiments were designed to measure osmotic activity after stopping tissue metabolism by heating. Bits of tissue removed from anesthetized dogs were placed in tared beakers containing water at $98^{\circ} \mathrm{C}$. After 30 minutes at $98^{\circ} \mathrm{C}$, freezing point measurements were made on supernatant fluid, and tissue osmotic activity was calculated from weights of beakers, water and dry tissues. Whole blood, liver, and kidney were hypotonic to serum by 20,10 , and $10 \mathrm{mOsm}$ per L., respectively. Losses of $\mathrm{CO}$, during boiling in whole blood, liver, and kidney were 15,8 , and $14 \mathrm{mM}$ per Kg., respectively. After correcting for $\mathrm{CO}_{2}$ loss, the freezing point depression of boiled tissue extracts became practically equal to that of serum. To evaluate the effect of dilution on activity coefficients of tissue electrolytes, the supernatant was concentrated until its observed freezing point depression was equal to that of plasma, and F. P. measurements were made at several dilutions. At the dilution range of most experiments, the change of activity coefficient of tissue electrolytes was only plus 3 per cent. These data imply: 1) that tissues are isotonic 
with plasma, if it is valid to extrapolate to conditions in the living cell; or 2) that liquid $\mathrm{N}_{2}$ and crushing may split protein or polypeptide chains in tissues, while boiling prevents splitting or promotes adhesion of such chains. Apart from data on erythrocytes, we are not aware of any other measurements on colligative properties of tissues in direct support of the classic concept of intracellular isotonicity.

Labelling of Leukocytes In Vivo with DFPa. J. W. Athens, A. M. Mauer, Helen Ashenbrucker, and G. E. Cartwright,* Salt Lake City, Utah.

Leukocytes have been successfully labelled with radioactive diisopropylfluorophosphate (DFP ). As a label, this compound has the following advantages: (1) the leukocytes can be tagged in vivo and thus the disadvantage of handling the leukocytes under non-physiologic conditions is avoided; (2) after an initial equilibration period DFP $^{22}$ is bound firmly and irreversibly to the proteins of the cell; (3) the label is promptly excreted from the body and not reutilized following the death of the cells; and (4) by the use of a plastic scintillation detector the radioactivity in the leukocytes isolated from a relatively small volume of blood $(20 \mathrm{ml}$.) can be measured simply, accurately and rapidly.

Two mg. of $\mathrm{DFP}^{82}$, containing 200 to $400 \mu \mathrm{c}$. of radioactivity, are administered either intramuscularly or intravenously. Thereafter, at appropriate intervals of time, leukocytes are isolated free of platelets and erythrocytes, plated between two plastic "Scintilon" squares and the amount of radioactivity determined. The activity is expressed as CPM per mg. of leukocyte nitrogen.

$\mathrm{DFP}^{22}$ has been administered to 14 normal subjects. The resulting radioactivity curve in the leukocytes consists of three components. (1) During the first two days there is a rapid decline in the activity. (2) From the second to about the tenth day, the activity remains relatively constant. (3) Thereafter, the activity again declines and is no longer detectable after $19.4 \pm 4$ days. Varying the initial dose of DFP ${ }^{82}$ between 1.0 and $4.0 \mathrm{mg}$. and altering the route of administration do not modify the shape of the curve.

Although interpretation of the complex curves is difficult in terms of leukocyte kinetics, the curves have been quite reproducible in normal subjects. This method should be extremely useful in studying the physiology and kinetics of leukocytes in normal and abnormal conditions.

Salicylates and Thyroid Function. F. K. Austen, M. E. Rubini, W. H. Meroney, and J. Wolff, Washington, D. C., and Bethesda, Md. (Introduced by Henry K. Beecher).

A hypermetabolic state associated with depressed thyroid function can be produced in euthyroid patients with rheumatoid arthritis by the daily administration of 7.5 grams of sodium salicylate. The BMR is raised to plus 30 to 60 per cent, serum cholesterol is depressed, and there is a profound creatinuria. Paradoxically, tachycardia, hyperkinesis, and heat intolerance are lacking, and the patients become lethargic without significant alterations in acid-base balance. The serum protein-bound iodine (PBI) gradually falls an average of 40 per cent over several weeks. The biological half-life of $\mathrm{I}^{131}$ thyroxine is reduced to an average of 4.0 days during treatment, and returns to the pretreatment average of 6.8 days upon cessation of therapy. In contrast to hyperthyroidism and certain other hypermetabolic states, in which the extrathyroidal pool of organic iodine is increased, the pools of salicylate-treated patients are diminished. Thyroidal $\mathrm{I}^{131}$ uptakes, at 2, 5, and 24 hours, are depressed an average of 30 per cent below pretreatment values. However, the patients respond normally to exogenous TSH as measured by an increase in $\mathrm{I}^{182}$ uptake, PBI, and further elevation in BMR. Rat thyroid slices incubated with sera of salicylate-treated patients $\left(3 \times 10^{-3} \mathrm{M}\right.$ salicylate) show no significant reduction in the capacity to incorporate iodide.

While the increased disappearance rates of labeled thyroxine could, in part, account for the lowered serum PBI, the expected compensatory thyroid hyperfunction fails to occur. A direct action of salicylates on thyroid function was not found by in vitro studies, and the capacity of the gland to respond to exogenous TSH is retained. These findings are analogous to those obtained in rats with 2,4-dinitrophenol, and suggest that salicylates not only increase metabolic rate by peripheral action, but depress thyroid function by inhibition of the production or release of TSH.

\section{Relationship of Calcium and Citric Acid to Hyperventila- tion in Man. D. Robert Axelrod, Brooklyn, N. Y.} (Introduced by Robert F. Pitts).

Neither $\mathrm{pH}$ change nor increase in protein bound calcium is sufficient to fully explain the decreased activity of calcium induced by hyperventilation. In this study, voluntary hyperventilation for twenty minutes produced arterial $\mathrm{pH}$ above 7.50 and $\mathrm{pCO}_{2}$ of $21 \mathrm{~mm}$. $\mathrm{Hg}$, or less. Frank tetany was not usually produced, but all subjects had marked paresthesias. This magnitude of hyperventilation produced essentially no change in plasma calcium concentration. Plasma citric acid levels rose, paralleled by $a$ fall in inorganic phosphate. Maximal changes occurred $\mathbf{4 0}$ to $\mathbf{5 0}$ minutes after the start of hyperventilation (20 to 25 minutes after cessation of hyperventilation). Plasma potassium levels did not change significantly. Arterial and venous measurements were made of glucose, citric acid, and inorganic phosphate. There was essentially no change in plasma glucose concentration, or glucose $\mathrm{A}-\mathrm{V}$ differences. A-V differences for phosphate were small and inconsistent, despite the fall in concentration. Citric acid $\mathrm{A}-\mathrm{V}$ differences indicated a contribution of citric acid from forearm tissue, and a trend of increased delivery after hyperventilation. Hyperventilation produced by 6 per cent $\mathrm{CO}_{2}$ inhalation did not alter plasma calcium, citric acid, phosphate or glucose concentrations. 
The findings of this study suggest that hyperventilation causes a loculation of calcium by metabolically produced citric acid, and that the changes in citric acid levels are a reflection of changes in intermediary metabolism. The time lag before plasma citric acid reached its maximum may be due to the decreased peripheral circulation during hyperventilation, and to the time required to establish equilibrium between cellular fluid and plasma. The increase in citric acid was as much as $3 \mathrm{mg}$. per cent, and plasma levels exceeded $5 \mathrm{mg}$. per cent. This magnitude of increase is sufficient to indicate that calcium is complexed with citric acid in hyperventilation, and this complexing may contribute to the paresthesias and tetany of hyperventilation.

Pulmonary Function in Asbestosis of the Lungs; $A n$ " $A l$ veolar-Capillary Block" Syndrome. MORTIMER E. Bader, Richard A. Bader, and Irving J. Selikoff, New York, N. Y. (Introduced by Alexander B. Gutman).

While the clinical and roentgenographic features of asbestosis of the lungs have received considerable attention in the past, the physiological abnormalities have not been as well defined. Pulmonary function studies in eighteen patients with asbestosis of the lung indicate that the primary pathophysiological defect is one of "alveolarcapillary block."

In all the patients, pulmonary function was evaluated by conventional tests and in five of these by measurement of the diffusing capacity of the lung for oxygen. All had a history of protracted exposure to asbestos dust and had varying degrees of typical roentgenographic involvement. All had dyspnea on exertion; other complaints included chest pain and cough.

Measurement of lung volumes revealed a well-preserved vital capacity, with moderate reduction in six cases and significant reduction in two. Total lung capacity was not significantly reduced. The ratio of residual volume to total lung capacity was less than 35 per cent in all but two cases. The maximum breathing capacity was well maintained. There was significant hyperventilation at rest and exercise in more than half the patients. The index of intra-pulmonary mixing was normal in all but one instance. Arterial oxygen saturation at rest was greater than 94 per cent in twelve patients, and 93 per cent in six. Following exercise, it was greater than 94 per cent in five patients, 90 to 92 per cent in five, 85 to 90 per cent in four, and less than 85 per cent in three. In five patients, diffusing capacity of the lung for oxygen was measured at rest by the method of Riley and Cournand and found significantly reduced in three; the calculated venous admixture was increased in three patients.

The findings of normal maximum breathing capacity, normal intra-pulmonary mixing, absence of emphysematous changes in lung volumes, arterial oxygen unsaturation on exercise, and impaired oxygen diffusing capacity indicate the presence of an "alveolar-capillary block" syndrome.
Physiology of Anemia in "Hypersplenism." Mario BaLDINI, Boston, Mass. (Introduced by William Dameshek).

Our previous experiments with methylcellulose "hypersplenic" rats, performed with the lactating rat technic, showed that the enlarged spleen produces a myeloinhibitory factor. Newborn rats lactated by hypersplenic mothers developed anemia, whereas anemia did not appear when the "hypersplenic" mothers had been previously splenectomized.

Recent experiments demonstrated that "hypersplenic" rats presented both increased hemolysis and bone marrow inhibition. Hemolysis was extracorpuscular in character and disappeared after splenectomy. Bone marrow inhibition was demonstrated using different types of hemolytic anemias (phenylhydrazine, immune serum) in parallel groups of rats. With equal degrees of anemia the reticulocyte values were always lower in "hypersplenic" anemia than in "pure" hemolytic anemias.

Further investigations were performed in selected human cases of "hypersplenism" in which the bone marrow showed no infiltration with abnormal cells. The rate of red cell production was estimated from the chromium red cell survival time, a valid estimation when the red cell count is stable.

The results obtained demonstrate that:

1. Increased hemolysis was present and was extracorpuscular in type;

2. Red cell production was increased, but the production index then determined remained in the range of 1.5 to 3, whereas in "pure" hemolytic anemias (autoimmune hemolytic anemia and hereditary spherocytosis) the production index was between 5 and 8;

3. Bone marrow production was never below 1 , so that after splenectomy, with the disappearance of the hemolytic factor, anemia also disappeared.

In human "hypersplenism," two factors combine to produce anemia: increased hemolysis and bone marrow depression. The latter is "relative" in character since the bone marrow is shown to be deficient only when peripheral need is higher than in the normal. It is not clear whether "hypersplenism" in humans is related to a factor produced by the cells of the spleen or to the fundamental pathologic process which also causes the splenomegaly.

\section{Exaggerated Natriuresis in Essential Hypertension.} David S. Baldwin, Albert W. Biggs, and William H. Hulet, New York, N. Y. (Introduced by Herbert Chasis).

Hypertensive subjects excrete more sodium than normotensive subjects in response to the infusion of hypertonic saline. The studies reported here indicate that the exaggerated natriuresis is probably initiated by extrarenal factors.

Observations on renal hemodynamics and on $\mathrm{Na}$, water, and total solute excretion were made in 22 hypertensive and 13 normotensive subjects after fluid deprivation and during the last 20 minutes of one-hour infusion of 2.5 per 
cent $\mathrm{NaCl}$ at 8 to $13 \mathrm{cc}$. per min. In normotensive subjects the $\mathrm{Na}$ excretion fraction averaged 2.3 per cent. In hypertensive subjects this fraction averaged 7.7 per cent. Exaggerated natriuresis induced by saline does not correlate with change in filtered load. Exaggerated natriuresis was also observed in hypertensive subjects during infusion of PAH and inulin in distilled water (2 cc. per min.), showing it does not depend on sodium or osmotic loading.

Basal $\mathrm{Na}$ excretion was comparable in the two groups, i.e., hypertensive subjects are not salt losers. With more advanced disease, the $\mathrm{Na}$ excretion fraction is correspondingly increased, maintaining $\mathrm{Na}$ balance. During $\mathrm{Na}$ deprivation hypertensive subjects conserve $\mathrm{Na}$ effectively.

An exaggerated natriuresis, similar to that observed in hypertensive subjects, was induced in normotensive subjects by prolonging the saline infusion to two or three hours (avg. excretion fraction 10.2 per cent). Conversely, exaggerated natriuresis in hypertensive subjects was abolished by salt deprivation (avg. excretion fraction 2.9 per cent).

Similar basal urine osmolalities were observed in hypertensive and normotensive subjects after fluid deprivation. During hypertonic saline infusion $T^{e}{ }_{\mathrm{H}_{2} \mathrm{O}}$ was comparable in the two groups. It is inferred that the concentrating mechanism is unimpaired in early hypertensive disease.

The absence of evidence of tubular impairment in hypertensive subjects and the fact that exaggerated natriuresis can be obtained without sodium or osmotic loading and is abolished during $\mathrm{Na}$ deprivation suggest that the natriuresis is initiated extrarenally.

The Relationship of the Pituitary to Hyperlipemia of Induced Nephrosis in Rats. Peter Bally and ShoSAKU NuMA, Boston, Mass. (Introduced by Kendall Emerson, Jr.).

Serum lipids were studied in 10 normal and 10 hypophysectomized male Sprague-Dawley rats weighing from 150 to 280 grams before and at four to six days after induction of nephrotoxic serum nephrosis. They were maintained on a Purina checker diet with white bread and raw carrots as a supplement. The onset of nephrosis was evidenced in all animals by massive proteinuria.

The serum levels of total cholesterol, phospholipid and total protein were determined. Lipoprotein distribution was studied with the preparative gradient density tube ultracentrifugation method of Oncley and Mannick. Albumin was determined by paper electrophoresis.

It was found that (1) beta lipoprotein distribution pattern of rat serum seemed identical to the human pattern as far as the location of protein peak concentration is concerned; (2) there is a marked rise of high density $(\mathrm{d}: 1.029-1.053)$ and low density $(\mathrm{d}:<1.026)$ beta lipoproteins in the nephrotic stage; (3) that in the rat lipemia is not necessarily a reciprocal function of albumin concentration. Hypophysectomized nephrotic rats with severe hypo-albuminemia developed only mild hyperlipemia as compared to control nephrotic rats with equal depression of serum albumin concentration; (4) preliminary studies indicate that cortisone acetate administered $(0.1 \mathrm{mg}$. per $100 \mathrm{gm}$. body weight per day, s.c.) to hypophysectomized nephrotic rats for two days prior to sacrifice will result in a partial but not complete restoration of the hyperlipemic response.

Similar studies of the hyperlipemic response to nephrosis in adrenalectomized rats are in progress.

An Attempt to Apply the "Cell Separation Theory" to Renal Hemodynamic and Metabolic Measurements in Intact Man. EARL S. BARKER and JoHN K. Clark,* Philadelphia, $\mathrm{Pa}$.

Pappenheimer and Kinter suggest that "plasma skimming" may separate red cells and plasma within the kidney. Data from over 50 human clearances and renal vein catheterization seem difficult to reconcile with a major regulatory role for such a process even if "cell separation" occurs. Plasma flow through the postulated "long" circuit supplying the peritubular capillary network was estimated by effective renal plasma flow (ERPF) less a small correction for para-aminohippurate (PAH) filtered in the cell-rich "short" circulation by-passing renal tubules. Total plasma flow (direct Fick) so divided in 26 normal subjects under control conditions averaged 557 and $52 \mathrm{ml}$. per min. for the "long" and "short" circulations.

Renal oxygen consumption $\left(\mathrm{Q}_{2}\right)$ has been described as flow limited by oxygen supplied the tubules. This concept applied to our $Q_{0_{2}}$ data appears to require unreasonable hematocrit values (low in the "long" circulation, high in the "short" circulation) and to contradict a highly significant inverse correlation between renal blood flow (RBF) and arteriovenous oxygen difference, demonstrable whether RBF increases or decreases. These data are not incompatible with cell separation itself. One might postulate that the concept of flow limitation of $\mathrm{QO}_{2}$ applies only to deeper nephrons, while the inverse correlation mentioned occurs in peripheral nephrons where more red cells reach the tubules.

Neosynephrine administration or head-up tilt decreases ERPF while PAH-extraction and filtration rate remain relatively constant. Cell separation could explain each change, but the reasoning invoking multiple compensating effects becomes so complicated that renal vasomotor changes seem a more attractive explanation.

\section{A Non-Renal Effect of Adrenal Cortical Steroids upon Potassium Metabolism. Frederic C. BARTter* and Paul Fourman, Bethesda, Md., and Cardiff, United Kingdom.}

When desoxycorticosterone-like steroids are administered, loss of potassium is among the well-documented sequelae. When ACTH and hydrocortisone-like steroids are administered, potassium loss also occurs. Two kinds of study were done in man to determine whether the second type of potassium loss has a mechanism similar to that of the first. 
In balance studies, it was shown that the second type, as opposed to the first, regularly appears on the first day, seldom lasts more than a day even when treatment is continued, precedes sodium retention, and is not prevented by sodium restriction. It also precedes loss of nitrogen.

In short-term studies it was shown that the second type of potassium diuresis, as opposed to the first, may be accompanied by a rise in serum potassium. Whereas with desoxycorticosterone-like steroids there is an increase in urinary titratable acidity and ammonium with a fall of $\mathrm{pH}$, with hydrocortisone-like steroids there is usually a decrease in urinary titratable acidity and ammonium with a rise of $\mathrm{pH}$.

Whereas the former phenomenon appears to result from a steroid-induced exchange of potassium and hydrogen for sodium ions at the renal tubular level, the latter appears to result from a release of potassium from cells, with the renal effects secondary thereto.

\section{Hyperlipidemia of Nephrosis. JAMES H. BAXTER,* How-} ARD C. Goodman, and Richard J. Havel, Bethesda, Md.

Serum lipids (fasting) were studied in 25 patients, age 1 to 65 years, with the nephrotic syndrome. Total cholesterol (TC), free cholesterol (FC) and phospholipid (PL) were elevated in all cases. Triglyceride (TG) was considerably elevated in 19 cases (with TC 354 to 1522), many of whom had lactescent serum. However, in 6 cases (with TC 460 to 675), TG was less than $200 \mathrm{mg}$. per $100 \mathrm{ml}$. and serum was clear. These studies together with chemical studies on lipoprotein fractions separated by ultracentrifugation indicated that the spectrum of lipoprotein abnormalities could be divided into three groups characterized respectively by the following patterns: (1) elevation of the density 1.019 to 1.063 fraction alone (in the six cases with clear serum noted above), (2) elevation of the $<1.019$ fraction alone (in cases with very high TG and milky serum), or (3) elevation of both these fractions. The pattern changed under observation in 2 cases. On the whole, the patients of group 1, while definitely hypoalbuminemic, had higher levels of serum albumin than most of the others. TC/PL of the lipoprotein fractions was often abnormal.

During remissions and exacerbations of nephrosis there was generally good inverse correlation between levels of serum lipids and level of serum albumin. To determine whether lipid levels are specifically related to level of serum albumin rather than to other parameters of the disease, three nephrotic patients were given courses of concentrated albumin intravenously. A fall in lipids occurred in all cases, though other changes in the disease state also occurred. During a period of more than 2 weeks in which a normal level of serum albumin was maintained in one patient (of group 1 above), serum lipids remained approximately normal. These observations support the concept that hyperlipidemia (hyperlipoproteinemia) of nephrosis is at least in part secondary to hypoalbuminemia.
The Metabolic Effects of Human and Monkey Growth Hormone in Man. J. C. BECK, E. E. McGarRy, I. Dyrenfurth, and E. H. Venning, Montreal, Quebec. (Introduced by J. S. L. Browne).

The diverse physiological effects of growth hormone in animals are well described but data on the effects observed in man are inconclusive. Support for the concept of species differences in the growth hormone was provided by Knobil who found monkey growth hormone to be physiologically active in monkeys. We wish to report the metabolic effects observed in a 13-year-old male pituitary dwarf during the administration of human and monkey growth hormone prepared by Raben. Both preparations of growth hormone resulted in a significant enhancement of nitrogen storage which was evident on the second day of its administration and which persisted after cessation of treatment. During the administration of both preparations of growth hormone there was a retention of potassium, phosphorus, calcium and sodium. The positive nitrogen, potassium, phosphorus and sodium balance was due to a decrease in the fecal as well as the urinary excretion of these substances. There was a fall in fecal calcium but an increase in urinary calcium during the growth hormone administration. A gain in body weight occurred during both periods and was maintained for four days after stopping the human material. There was a significant increase in urinary aldosterone excretion during both periods of growth hormone administration. This was most marked with the human growth hormone and was not accompanied by any alteration in urinary 17-hydroxycorticoid and 17-ketosteroid excretion. Impairment of the glucose tolerance curve was evident after 10 days of human growth hormone administration while no change was demonstrable on the fifth day of monkey growth hormone. The effects of these preparations on the serum electrolytes, calcium, phosphorus, alkaline phosphatase, urea, urinary-alpha-amino nitrogen and urinary vitamin $\mathrm{C}$ will be described. Further studies with human growth hormone are in progress.

\section{Kinetics of Reaction Between Insulin and Insulin-Binding Antibody. Solomon E. Berson * and Rosalyn S. YALOW, New York, N. Y. \\ Employing crystalline beef insulin- $\mathrm{I}^{131}$ as a tracer,} steady state and transient state kinetics of the reaction between beef insulin and insulin-binding antibody have been studied with antisera from insulin resistant and nonresistant subjects. Insulin and antibody react to form a nonprecipitating complex which is in reversible equilibrium with the uncomplexed species. Steady state studies indicate that insulin is univalent in the reaction but that two distinct orders of antibody binding sites are demonstrable in most antisera. The equilibrium constants are of the order of $10^{8}$ and $10^{\circ}$ liters per mole, respectively. Transient state studies are in agreement and reveal two single order dissociation constants averaging about 0.005 $\min .^{-1}$ and $0.2 \min ^{-1}$, respectively.

The demonstration of cross reactions between beef and pork insulin in these studies has suggested that the two 
orders of antibody binding sites observed may be due to different affinities of each of the species specific antibodies for beef insulin.

Maximal capacity for insulin binding rarely exceeded 10 units insulin per liter plasma in antisera from nonresistant subjects but ranged from 80 units per liter to more than 400 units per liter in the sera of four subjects with insulin resistance. These studies suggest an explanation for the so-called "insulin unresponsiveness" manifested by some insulin-treated subjects on the basis of rapid binding of administered insulin by antibody. Furthermore, the tendency of insulin-resistant subjects to develop manifestations of hypoglycemia for a number of days following cessation of high dosage insulin therapy is consistent with the slow dissociation of insulin from the insulin-antibody complex observed in these subjects; the insulin-antibody complex serves as a source of "depot" insulin which is released in significant quantities over a prolonged period of time.

An immuno-assay method for the determination of insulin concentrations in body fluids has been developed on the basis of these studies.

The Effect of the Administration of $\mathrm{HN}_{\mathrm{s}}$ on the Utilization of Plasma Iron in Humans. AlBert BertinChamps, Y. Kenis, and Henry J. TAgnon,* Brussels, Belgium.

The administration of methyl-bis ( $B$ chloroethyl) amine hydrochloride $\left(\mathrm{HN}_{\mathrm{z}}\right)$ in therapeutic doses often produces a transient leukopenia resulting from an injurious effect on the hemopoietic system. However, little or no anemia is produced by a single injection of 0.2 to $0.4 \mathrm{mg}$. of $\mathrm{HN}$, per Kg. of body weight. Therefore, the effect of $\mathrm{HN}_{2}$ on erythropoiesis may not be apparent from observations of the peripheral blood. This effect was investigated by studying the turnover of plasma iron daily by means of radioactive iron after the administration of 0.2 or $0.4 \mathrm{mg}$. per $\mathrm{Kg}$. of body weight of the drug. Eight patients with cancer were studied who had never been treated before and had not received blood transfusions.

Plasma iron turnover was normal before the injection of $\mathrm{HN}_{2}$. After the injection, there was a profound depression of turnover reaching a peak between the third and the fourth days and returning gradually to normal between the tenth and the fifteenth days. The maximum depression was of the magnitude seen in cases of aplastic anemias.

These results show that $\mathrm{HN}_{2}$ in our patients had a pronounced effect on hemoglobin synthesis although such effect did not necessarily express itself in significant changes in peripheral blood values: these remain normal because the depression of erythropoiesis is transient and the red cells do not have a much shortened life span.

The fact that the maximum effect of turnover takes place on the second to fourth days after a single dose of $\mathrm{HN}_{2}$ cannot be explained presumably by a persistent action of the drug, since the cytotoxic action of $\mathrm{HN}_{2}$ is apparently completed within a few minutes. The delay may represent the time interval separating the stage of erythropoiesis on which $\mathrm{HN}_{2}$ has maximum activity and the stage at which hemoglobin is maximally incorporated.

Factors Affecting the Appearance of Ammonia in the Gastric Juice. S. P. Bessman and J. C. Staurfer, Baltimore, Md. (Introduced by Theodore E. Woodward).

The relative permeability of molecular ammonia in comparison to ammonium ion has been proposed as a major factor in the toxicity of circulating ammonium ion. This phenomenon has also been evoked in studies on the mechanism of the toxicity associated with alkalosis. Since a major factor in all of these calculations is the relative $\mathrm{pH}$ on both sides of the membrane, a study was made of the $\mathrm{pH}$, ammonia, and urea nitrogen content of arterial blood and of the gastric juice of patients during the course of intravenous infusion of one millimol of ammonium chloride per kilogram in one hour. A lag was found between the rise in blood ammonia and the appearance of an increase in ammonia of the gastric juice. The increase of ammonia could be correlated with the increase of urea in the blood, confirming the observations of Von Korff on the relation of blood urea nitrogen to gastric ammonia. The additional information derived is that there is no correlation between gastric $\mathrm{pH}$ and the appearance of ammonia in the stomach. This was studied by adding sufficient bicarbonate to the stomach contents to maintain neutrality. There was no change in distribution of ammonia between gastric juice and blood. The conclusion is drawn that the ammonia of gastric juice is present solely as the result of urease activity, and is not in equilibrium with the blood.

\section{Iron Enzymes in Iron Deficiency: Cytochrome C. ER- NEST Beutler, Chicago, IIl. (Introduced by Wright Adams).}

It is well known that changes in the fingernails, mouth, and esophagus occur in iron deficient patients, and it has been claimed that they may occur in the presence of normal peripheral blood counts. The symptoms of patients with iron deficiency often are greatly out of proportion to the degree of anemia. They may disappear within a few days after the institution of iron therapy, before any hematologic response has occurred. These observations could be explained by changes in iron-containing intracellular enzymes in iron deficiency, but it is commonly believed that the iron-enzymes are not affected by iron deficiency. The origin of this firmly ingrained concept is difficult to trace, but it may be based on the claim by Hahn and Whipple that "muscle parenchyma iron are inviolate stores of iron which are not drawn upon no matter how great is the emergency due to anemia." Careful quantitative determinations of iron enzymes in the tissues of iron deficient animals have never, to our knowledge, been reported. As a model, we have chosen cytochrome $\mathrm{C}$, because of its biological importance and because it is possible to measure the concentration of this heme enzyme spectrophotometrically entirely independently of hemoglobin. Rats were made iron deficient 
by bleeding while receiving a diet that was well balanced except for a very low iron content. Control rats were bled in the same manner and received an identical diet except for the addition of an iron salt. A spectacular decrease in the concentration of both liver and kidney cytochrome $\mathrm{C}$ was observed in the iron deficient group, the decrease greatly exceeding the decrease in hemoglobin. The widely held concept of the "inviolate nature" of tissue iron enzymes therefore has proved to be false.

Stimulation of the Bone Marrow: A Clinical Study in Man. H. R. Bierman,* K. H. Kelly, R. L. Byron, JR., H. Ratrunde, and F. Cordes, Duarte, Calif.

The increasing incidence of hematopoietic depressant states due to radio-activity, medication or of idiopathic origin has emphasized the critical need for data concerning leukopoiesis and, in particular, granulocytopoiesis and methods for stimulating the bone marrow. Previous data have shown that leukopoiesis follows many of the basic physiological laws of erythropoiesis. Consequently the response of the bone marrow to the rapid removal of 11 to $88 \times 10^{\circ}$ leukocytes was studied in 14 patients with various neoplastic diseases.

Serial bone marrow aspirates initially showed a marked depletion of all marrow elements which persisted for 4 to 24 hours as compared with that of the control samples. Immediately afterwards increased granulocytopoiesis and megakaryocytosis appeared with numerous immature forms and the marrow rapidly became hypercellular. Erythropoiesis was similarly stimulated although the hematocrit was maintained between 38 to 45 . Leukopoietic activity persisted for 24 to 96 hours during which time increasing maturation of the leukocytes and erythrocytes was observed and increased numbers of immature megakaryocytes made their appearance on the fourth day. The marrow returned to the control pattern within the next 4 to 7 days. The degree and duration of marrow stimulation appeared to be related to the total number of leukocytes removed. Subsequent leukocyte withdrawals showed greater leukopoietic response than initially.

The events of leukopoiesis, megakaryocytosis and erythropoiesis occurred in a well defined chronological order in normocellular, hypercellular, and leukemic marrows. The abrupt hematopoietic stimulus permitted estimates of maturation times and life spans of the various granulocytic forms in the marrow. The effects of hematopoietic stimulation upon the biochemical and hematologic constituents of the circulating blood were related to the degree of leukopoiesis. The significance of these studies to repopulating the marrow and the chemotherapeutic index will be discussed.

Serum Acid Mucopolysaccharides. Alfred Jay Bollet, Maria W. Seraydarian, and Rachel E. TUrner, Detroit, Mich. (Introduced by Gordon B. Myers).

Mucopolysaccharide containing uronic acid has been isolated from serum by a method suitable for quantitation.
It contains two components staining metachromatically with toluidine blue. Both are hyaluronidase-labile. On paper chromatography, one has mobility in two systems resembling chondroitin sulfate and was found to have a molar ratio of hexosamine to uronic acid of 1.1 to 1 . The second has a chromatographic mobility resembling heparin and beta-heparin (chondroitin sulfate B), but differs from both in being hyaluronidase-labile. Further characterization is in progress. No component with the chromatographic mobility of hyaluronic acid has been found in serum, although such material is demonstrable in pleural, pericardial and ascitic fluids. These fluids also contain the two chromatographic components found in serum, although the total concentration of acid mucopolysaccharides is usually lower than in serum obtained simultaneously.

Sera from 34 normal individuals were found to have a mean level of $277 \mathrm{mcg}$. per cent (S.D. \pm 0.042 ) acid mucopolysaccharide measured as glucuronic acid (carbazole method of Dische). Elevated levels were found in a majority of 55 cases of rheumatoid arthritis, 7 cases of rheumatic fever and 8 cases of systemic lupus erythematosus. Occasional cases with non-rheumatic diseases also showed elevations. Increased levels were not specific for any disease, but were not related to the presence of inflammation in the manner of an acute phase reactant. Chromatographic studies of sera from patients with several rheumatic diseases revealed the same two components found in normal sera.

The Handling of Erythrocyte Iron by the Reticulo-Endothelial Cell. T. Botru wert, W. D. Noyes, and C. A. Finch,* Seattle, Wash.

The present studies involve the administration of radioiron-tagged erythrocytes rendered non-viable by storage at $4^{\circ} \mathrm{C}$ in sodium citrate for 24 to 28 days to normal subjects and patients with pertinent disease. The clearance of these cells from circulation, the change in plasma iron concentration and the plasma iron turnover were subsequently measured. Utilizing a double isotope technique $\left(\mathrm{Fe}^{\mathrm{s}}, \mathrm{Fe}^{\mathrm{ss}}\right)$, the amount of iron remaining in the reticulo-endothelial cell and the amount made available to the marrow were determined. In vivo distribution of iron was estimated by external body counting.

Studies with varying loads of non-viable cells indicated a maximal clearance rate from circulating blood of about $0.5 \mathrm{ml}$. RBC per hour per $\mathrm{kgm}$. body weight by normal subjects. Plasma iron levels rose to saturation within 1 hour but plasma iron turnover remained unchanged. In normal subjects approximately one-third of a $1 \mathrm{mg}$. per $\mathrm{kgm}$. dose of erythrocyte iron was metabolized by the reticulo-endothelial cells and passed on to the marrow without significant delay while the remainder stayed in a slow-moving pool as cellular stores. There was little effect of prior saturation of the plasma iron on this mechanism and there was no specific body localization of erythrocyte iron, the greatest amount appearing in the liver.

In patients with iron deficiency and in pernicious 
anemia responding to therapy there was increase in plasma iron turnover with rapid and complete utilization following infusion of non-viable erythrocytes. In infection and iron storage diseases there was little change in plasma iron levels or its turnover, most of the iron remaining in storage.

Thus the reticulo-endothelial system has a finite capacity to process non-viable erythrocytes. The amount of iron stored or delivered to the plasma depends on the dose administered, the needs of the erythroid marrow and the functional integrity of the reticulo-endothelial cell.

Type Specific Antibody Response to M-Protein of Nephritogenic Streptococci in Glomerulonephritis. A. I. Braude,* Mary Bone, Billie Waggoner, and Betty Haten, Dallas, Tex.

The predominance of type 12 streptococci among patients with acute glomerulonephritis led Rammelkamp to postulate that certain streptococcal types are nephritogenic. If his hypothesis is valid, patients with glomerulonephritis should develop type-specific antibodies to M-protein of nephritogenic streptococci. Studies were therefore undertaken to demonstrate such antibodies in an effort 1) to secure immunologic evidence supporting the thesis that nephritogenic streptococci exist; 2) to investigate the role of nephritogenic streptococci in numerous nephritics from whom nontypable or no streptococci were isolated; and 3) to account for immunity against recurrences of glomerulonephritis.

Type specific antibody was measured against type 12 , and the provisional Red Lake type by bacteriostasis, mouse-protection, and complement fixation. Blood was examined from: 1) 54 patients on the Red Lake Indian Reservation two years after recovery from epidemic glomerulonephritis ; 2) 56 patients with sporadic glomerulonephritis in Dallas.

The simultaneous performance of three tests disclosed antibody in numerous bloods when one of the three tests was negative. Among sporadic cases, 70 per cent of sera subjected to three tests contained antibody to type 12 , and 25 per cent to Red Lake; conversely, 91 per cent of sera from Red Lake Indians contained Red Lake antibody and 30.6 per cent antibody to 12 . Among normals, only 9.8 per cent contained antibody to 12 and 7.7 per cent to Red Lake. Type-specific antibody was detected from ten days to 4.5 years after onset of nephritis, but failed to develop if penicillin had been given for acute streptococcal infections. The frequent occurrence of antibodies simultaneously to both nephritogenic types suggested a common antigenic relationship.

These results support the bacteriologic evidence for the existence of nephritogenic streptococci by demonstrating almost universal presence of type-specific antibody to nephritogenic streptococci in persons recovered from acute glomerulonephritis. They also account for immunity to recurrent glomerulonephritis caused by both homologous nephritogenic types and heterologous nephritogenic types sharing related antigens.
Hemodynamic Effects of Quantified Mitral Regurgitant Flow. E. Braunwald, G. H. Welch, JR., S. J. SarNOFF, and A. G. Morrow, Bethesda, Md. (Introduced by S. S. Kety).

A more precise appreciation of the acute hemodynamic consequences of mitral insufficiency was obtained in the dog by producing it in such a manner that the regurgitant flow could be controlled and measured, while simultaneously measuring effective cardiac output, left atrial, left ventricular and aortic pressures. This was accomplished by permitting blood to flow during systole from left ventricle into the left atrium through an external circuit containing a flowmeter. Regurgitant flows up to three times basal cardiac output were tolerated with minimal alterations in forward flow, aortic, left atrial and ventricular pressures. Such regurgitant flows produced only slight depression of effective left ventricular function curves. Any given increase in regurgitant flow required substantially smaller increments in ventricular filling pressure than did similar increases in forward flow, suggesting that ventricular emptying is more complete in the presence of mitral insufficiency. With any given mitral regurgitant orifice, elevating aortic pressure increased regurgitant flow. It was also observed that the left atrial pressure rise brought about by increasing aortic pressure was a function of the mitral regurgitant orifice size. These latter observations were extended to a study of ten patients with mitral valve disease. During transbronchial left heart catheterization, acute elevation of systolic aortic pressure $(35$ to $85 \mathrm{~mm}$. $\mathrm{Hg}$ ) induced by infusions of nor-epinephrine markedly raised the left atrial " $v$ " wave of the three patients in whom clinically significant mitral insufficiency was present. In contrast, this effect was not observed in the seven patients with surgically demonstrated pure mitral stenosis. It is suggested that this difference in response can be helpful in the detection of mitral insufficiency, even in the presence of associated mitral stenosis.

Unilateral Renal Disease in Dogs: Method of Induction and Serial Bilateral Renal Function Studies. NeAL S. Bricker, Harvey R. Bernard, and James M. Stokes, St. Louis, Mo. (Introduced by Robert E. Shank).

Many aspects of the functional capacity of the kidney with chronic Bright's disease remain unexplained due to inability to relate physiologic data to control observations of the same subject. Experiments have therefore been undertaken to induce chronic renal lesions in a single kidney of dogs, preserving the integrity of the contralateral kidney. In such preparations, observations may be recorded on the simultaneous behavior of a diseased and normal kidney exposed to the same stimuli, in a common internal environment and with glomerular filtrates of identical composition.

After a bladder-splitting procedure to provide each kidney with a permanent hemibladder, two control clearance studies were performed in each animal. Thereafter a nephrotoxic aminonucleoside (2-6 dimethyl aminopurine 3 amino d-ribose) was infused into one renal artery (over 
approximately 10 minutes) while renal venous blood was diverted to prevent recirculation. Blood volume was maintained by infusion of dog whole blood.

Permanent unilateral depression in renal hemodynamics occurred, varying from moderate to severe in degree. Values in the normal kidney invariably increased and RPF occasionally doubled within 16 hours.

When impairment in GFR was marked both the concentration and the absolute amount of sodium in the urine were greater from the diseased kidney despite its lowered filtered loads. During hypertonic saline infusion the percentage of filtered sodium excreted was considerably greater on the diseased side. When GFR was only moderately depressed, however, the diseased kidney in some instances excreted proportionally less sodium than the contralateral organ.

Bilateral renal biopsies were performed before and after aminonucleoside injections. To date there has been no consistent lesion, although in one animal exhibiting marked functional depression, the kidney became contracted and showed dilated tubules containing casts.

Studies are in progress to evaluate the exact role of the aminonucleoside (as well as other nephrotoxic substances) in the genesis of the renal lesion.

Sensitivity to Buffy Coat-A Cause of "Non-Specific" Transfusion Reactions. T. E. BrItTINGHAM and $\mathrm{H}$. Chaplin, JR., St. Louis, Mo. (Introduced by C. V. Moore).

Patients requiring repeated blood transfusions sometimes develop increasingly frequent and severe febrile reactions to the infused blood. The reactions are generally considered to be of "non-specific" origin because of inability to demonstrate contamination of the blood, presence of pyrogens, red cell incompatibility, or sensitivity to the donor's plasma. The authors have repeatedly observed in the serum of such patients the presence of agglutinins against donor leukocytes, and sometimes against donor platelets. Patients have been observed to develop leukoagglutinins coincident with the onset of recurrent transfusion reactions.

The present report describes transfusion studies on a series of five patients exhibiting the coexistence of repeated "non-specific" transfusion reactions and demonstrable leukoagglutinins. Prior to each transfusion the donor's blood was partitioned into two fractions-(a) buffy-coat-"poor" (75 per cent of the total red cells, 65 per cent of the total plasma, $<10$ per cent of the total buffy coat) and (b) buffy-coat-"rich" (25 per cent of the total red cells, 35 per cent of the total plasma, $>90$ per cent of the total buffy coat). Minimal or no reactions were observed following transfusion of the buffy-coat"poor" fractions. Characteristic reactions (shaking chills, fever) followed transfusion of the buffy-coat-"rich" fractions. There were many similarities to the reactions which customarily follow the injection of bacterial pyrogens: (a) latent period of 60 to 90 minutes following administration, (b) characteristic chills and fever pattern, (c) immediate transient leukopenia followed by more sustained leukocytosis, (d) evidence of a "dosage" effect, and (e) suggestion of "tolerance" following repeated exposure. No reactions were observed during comparable studies on control subjects.

Buffy-coat-"poor" transfusions have been successfully employed in the management of several seriously ill reaction-prone patients. Methods for preparing buffycoat-"poor" blood for transfusion will be described.

The authors conclude that the leukocyte is an effective antigen and that isoimmunization to leukocytes is a common cause of recurrent febrile transfusion reactions.

Paper Electrophoresis and Gel Diffusion of Sputum in the Diagnosis of Pulmonary Carcinomatosis; Identification of the Fast Moving Component as Albumin. Samuel C. Burantz * and Aline W. Berns, St. Louis, Mo.

During our pursuit of immunochemical investigations of asthmatic sputum Warfvinge's observations on electrophoresis of sputum was published (Acta med. Scandinav., 1955, 153, 49). He subjected to paper electrophoresis sputa from 100 unselected cases of bronchopulmonary disease. Only a case of bronchiolar carcinoma revealed great increase of a protein component migrating like albumin. Sputum from a patient under our observation whose clinical course resembled bronchiolar carcinoma revealed a similar component on electrophoresis. Necropsy established her pulmonary nodules as metastatic from a primary carcinoma of the stomach. The incidence of this protein in sputum was then ascertained in a blind study. Sputa submitted to the pathologist for cytology were subjected to filter paper electrophoresis at 150 volts and 7.5 to 8.5 milliamperes for 16 hours in veronal buffer at $\mathrm{pH} 8.6$, ionic strength 0.075 . One hundred and four sputa from 58 subjects were studied and large amounts of the fast component detected in 11 cases. Five were proven pulmonary carcinomata. A positive diagnosis was not possible in 6 cases, but all presented pulmonary densities and are being followed. Paper chromatograms of hydrolysates of an eluate of the component yielded the amino acid pattern common to proteins. Gel diffusion with sputa and rabbit antisera to fractionated human serum proteins yielded within 18 hours intense bands of precipitate with the anti-albumin serum. This technique, more sensitive than paper electrophoresis and simpler to perform, is being employed with a larger series of sputa and with cervical mucus. Studies are in progress to ascertain the cellular origin of the albumin, traces of which appear in sputa of some apparently non-carcinomatous subjects. Notable is the absence of the component on electrophoresis of grossly bloody sputa or bronchial washings of non-carcinomatous subjects.

Some Psychopharmacological Effects of Atropine. ENoch Callaway, III, Raymond I. Band, and Jacob E. Finesinger,* Baltimore, Md.

The effects of the intramuscular injection of 2-mg. doses of atropine were studied in a series of twenty-one normal male volunteers. The personalities of the sub- 
jects were evaluated and they were also studied before and after injections of both atropine and saline. The studies included interviews, questionnaires, the Minnesota Multiphasic Personality Inventory, The Progressive Matrices, the Stroop Test, the Luchens Water Jug Test, and the Gottschaldt Test. The results are described in terms of 1) a peripheral sympathomimetic response, 2) a subjective reaction to the central effects of the drug, and 3) the effects of the drug on the subject's level of awareness. The performance of these subjects indicates that atropine in these doses facilitates the performance of a task requiring a broad focus of attention, and interferes with tasks which demand a narrower focus of attention.

Carbohydrate Metabolism as a Measure of Human Platelet Preservation. EDMUNd W. CAMPBeLL and Edith V. Lo Pilato, Boston, Mass. (Introduced by Samuel Proger).

The preservation of human blood platelets has been evaluated in vitro from study of the carbohydrate metabolism. Control values were compared with metabolic studies on platelets modified by the addition of certain metabolic substrates and by temperature changes.

Platelet-rich plasma was stored in silicone-coated containers at $4^{\circ} \mathrm{C}$ and minus $16^{\circ} \mathrm{C}$; substrates were added before and during storage. Radioactive phosphorus was added to some samples to determine the rate of phosphorus turnover. The platelets were separated, the glycolytic esters extracted with trichloracetic acid, and the nucleotides, sugar phosphates, etc., separated by anionexchange chromatography. Phosphorus was expressed in micrograms per $10^{10}$ platelets; radioactivity was determined by direct G-M assay.

The addition of adenylic acid, adenosine, adenosinediphosphate, and adenosine-triphosphate, apparently preserved metabolic activity, since nearly normal amounts of cytidilic acid, adenosine-monophosphate, adenosinediphosphate, adenosine-triphosphate, ribose and the sugar phosphates were recovered as compared with control values. A moderate increase in inorganic phosphorus was noted but the total phosphorus and per cent recovery were essentially normal. The addition of bivalent metals (e.g., magnesium, manganese) caused similar effects whereas monovalent metals (e.g., zinc) were much less effective. Thus, the addition of some purine nucleosides almost completely preserved metabolic activity; the addition of pyrimidine nucleosides, nucleotides and bivalent trace metals exerted a similar but less pronounced effect. This is contrary to studies reported on erythrocytes, suggesting that platelets may lack a typical cell membrane or manifest a different response to metabolic substrates. The effect of different additives was not necessarily universal throughout the cycle, nor were identical modes of activation present, but definite patterns were established.

A new approach to the problem of platelet preservation has been presented and current studies indicate that correlated investigation of platelet metabolism, physiologic function and in vivo viability may help to resolve this problem.

The Effect of Wine in Decompensated Hepatic Cirrhosis. John Carbone, Victor Sborov,* Rudolph Fanska, and Kathry Ringgold, San Francisco, Calif.

Alcoholic beverages in ordinary amounts have not been considered to be hepatotoxic to the normal liver. The use of alcohol even in small amounts, however, has been thought to be contraindicated in all forms of liver disease. Experimental data using human subjects to support this latter concept are not available.

To evaluate the effect of alcohol upon the diseased liver, five patients with advanced decompensated nutritional cirrhosis were carefully studied on a metabolic ward. Each patient was used as his own control. The experiment consisted of three twenty-one day periods. A daily basic diet consisting of 120 grams of protein, 300 grams of carbohydrate and 150 grams of fat was used. In the first study period the basic diet was supplemented with a daily ration of $1,000 \mathrm{cc}$. of Zinfandel wine (750 calories). In the second period the wine was withheld and the diet was maintained isocaloric with $1,000 \mathrm{cc}$. of sweetened fruit juice. The wine was again administered in the third period.

Liver biopsies were taken at the beginning of each period and at the end of the study. Serial liver function and water, electrolyte and nitrogen balances were measured throughout the study.

Despite the severe liver disease present at the beginning of the study, the administration of the wine did not seem to interfere with normal and rapid convalescence. The rate of clinical recovery was greatest in the first test period and gradually plateaued thereafter. No acceleration of recovery was noted when the wine was discontinued. The liver biopsies showed a high fat content initially which cleared rapidly with the passage of time and did not seem to be retarded by wine ingestion. All patients made a complete and uneventful clinical recovery.

These data do not support an hepatotoxic effect of alcohol in the presence of an otherwise adequate diet.

\section{Correction by Normal Platelets of Deficient Generation} of Thromboplastin in a Family of Hemophilics. R. T. Carroll and R. R. Holburn, Philadelphia, Pa. (Introduced by L. M. Tocantins).

A family of hemophilics has been studied in whom the principal defect in the blood was in the platelets. The defect was observed only in males and transmitted by females. Most of the affected males lived beyond the third decade, had little deformity of joints, and most of their bleeding episodes came only after operative procedures or severe injury. The coagulation time of venous blood was prolonged in glass and silicone tubes; recalcified plasma mixtures at 90 per cent concentration were incoagulable. Clot retraction, bleeding time and platelet count were normal. Prothrombin and Ac-globulin concentrations were slightly decreased, other coagu- 
lation factors were unchanged. Platelet cofactor activity of the patients' plasma was greater than normal. Addition of normal platelets restored the coagulation time of the patients' plasma to normal, while equal concentrations of the patients' platelets did not. The patients' platelets and serum were insufficient to induce complete thromboplastin generation in normal plasma. Normal platelets restored to normal the thromboplastin generation system containing patients' plasma and serum. Adding equal amounts of patients' plasma to Hemophilia A or B plasma of moderate grade resulted in normal clotting times. Patients' plasma did not prolong the coagulation of normal plasma. Intravenous injections of $100 \mathrm{ml}$. of platelet-poor normal plasma, either in single or multiple daily infusions, caused no change in the patients' venous blood clotting time, plasma hypocoagulability or rate of thromboplastin generation. One infusion of $100 \mathrm{ml}$. of platelet-rich normal plasma $\left(4.4 \times 10^{\circ}\right.$ platelets per cubic millimeter plasma) caused a 30 per cent rise in the platelet count, and a return of the venous blood clotting time, plasma clotting time and thromboplastin generation to normal limits for at least 3 days.

Renal Handling of Glucose in the Dog. Francis P. Chinard,* W. Rowland Taylor, Mary F. Nolan, and Theodore EnNS, Baltimore, Md.

Under ordinary circumstances the removal of glucose from the urine is sufficiently complete to prevent the escape into the bladder of any significant fraction of the glucose crossing the glomerular barrier. Evidence is outlined below indicating that glucose is transported across the tubule cell without breakdown and resynthesis of the 6 carbon chain and that glucose does not contribute significantly to renal carbon dioxide production. Injection is made nearly instantaneously into the renal artery of solution containing a reference substance, $\mathrm{T}-1824$ usually, creatinine and $1-\mathrm{C}-14$ glucose. Blood is collected through a catheter in the renal vein; 30 samples are obtained in a total period of 30 seconds to two minutes. The concentration of each of the injected substances is determined in each sample and is divided by the concentration in the injection mass to permit calculation of the relative recoveries. It is assumed that there is no loss of T-1824 during this single passage through the kidney. In 11 experiments the average recovery of creatinine was 76 per cent (the remainder being recoverable in urine); the recovery of C-14 was 98 per cent (insignificant amounts were found in the urine). That the C-14 activity resided in glucose was shown by autoradiography of paper chromatograms on which glucose had been identified chemically and enzymatically (glucose oxidase). As shown by microbiological and chemical degradation there was no randomization of the radioactivity originally present in the 1 position. In other experiments, renal venous blood samples were obtained anaerobically and the C-14 activity of the carbon dioxide determined. Uniformly labelled glucose contributed less than 0.1 per cent of its activity to the carbon dioxide. Carbon 14 labelled lactate contributed significantly.
Inducible (Adaptive) Stimulation of Staphylococcal Penicillinase In Vivo. Sidney CoHen and LippMan H. Geronimus, Boston, Mass. (Introduced by Benjamin Alexander).

Penicillin resistance in pathogenic strains of staphylococci is mediated by the enzyme penicillinase. The level of penicillinase activity in different strains appears to determine the degree of resistance to penicillin, as measured by conventional sensitivity tests. However, little information is available as to whether the penicillinase activity of an individual strain is fixed or may be altered significantly by environmental changes comparable to those encountered by the staphylococcus within its animal host.

In earlier in vitro experiments, we have shown that staphylococcal penicillinase activity may be increased 10 to 30 fold by exposure of staphylococci to penicillin under conditions allowing little or no bacterial multiplication. This increase in activity requires a complex medium and oxygen and is inhibited by Chloramphenicol or 8-hydroxyquinoline. These properties are consistent with inducible (adaptive) stimulation of penicillinase biosynthesis.

An analogous stimulation of penicillinase activity has now been demonstrated in vivo. Renal infections were produced in mice by the intravenous injection of a penicillinase-producing strain of staphylococcus. Half of the mice were given 500 units of procaine penicillin $G$ intramuscularly 36, 48, and 60 hours after infection. Seventytwo hours after infection, the animals were sacrificed. The kidneys were homogenized and the staphylococci were harvested, without opportunity for further multiplication, by differential centrifugation in sucrose and $\mathrm{KCl}$ solutions at $0^{\circ} \mathrm{C}$ and their penicillinase activity assayed. The staphylococci from penicillin-treated animals inactivated penicillin 8 times as rapidly (480 units of penicillin $/ 10^{s}$ staphylococci/hour) as organisms from untreated animals (61 units of penicillin $/ 10^{8}$ staphylococci/hour). Additional experiments gave no indication of selection of resistant mutants to explain these results. This evidence supports the hypothesis that, in animal infections, staphylococcal penicillinase may be increased inducibly by penicillin treatment.

Studies on Kinetics and Mechanisms of Potassium Transfer in In Vivo Skeletal Muscle. Hadley L. Conn, JR., and Jон N C. Wood, Philadelphia, Pa. (Introduced by Calvin F. Kay).

The quantitative kinetics and particularly the mechanisms responsible for transcellular potassium transfer in skeletal muscle have been little explored. It has become possible, with the aid of tracer methods, to study kinetics of transfer in in situ but isolated muscle. Results obtained under different experimental conditions permit insight into mechanisms involved. In the present studies, dog muscle was perfused with arterial blood containing radiopotassium, and the manner of approach to specific activity equilibrium of the venous blood and the muscle 
was analyzed mathematically to give potassium transfer constants. With additional data on non-radioactive potassium in blood and muscle and on inulin space, transfer rates could be computed. Transfer rates were obtained for resting muscle, contracting muscle activated by nerve stimulation, for muscle subjected to decalcification, and for muscle perfused with blood containing high glucose and insulin concentrations. In resting muscle the transfer rate was $0.4 \mathrm{mEq}$. per $\mathrm{Kg}$. (wet weight) per min. The same value was obtained for contracting muscle, and almost the same in the glucose-insulin studies. However, the latter showed a marked uptake of glucose and potassium by muscle, in a mole ratio of four to one, respectively. Decalcification produced a significant increase in potassium rate constants and transfer ratio. Cell potassium concentration was subnormal.

From these results the following tentative conclusions are drawn. In skeletal muscle 1) the cell membrane effects produced by flow of electrical currents, unlike in nerve, are not a significant factor in potassium transfer, 2) potassium transcellular transfer does not occur in association with glucose transport into the cell, but potassium uptake is increased by hyperglycemia because of subsequent glycogen storage and potassium "binding," 3) calcium deficit permits an increased cellular outflux of potassium compatible with the concept that calcium complexes help to maintain the "charged pore" integrity and thus the electrochemical gradient across the cell membrane.

\section{A Method for Serial Cardiac Output Determinations Ap-}

plied to the Study of Pregnancy Associated with Heart

Disease. Robert W. Cornetr and James Metcalfe,

Boston, Mass. (Introduced by C. Sidney Burwell).

The technique of cardiac catheterization is for practical purposes inapplicable to making numerous serial cardiac output determinations in individual patients.

An alternative method, which is applicable to serial use, consists of using the lungs as an aerotonometer and $\mathrm{CO}_{2}$ as the indicator gas. The $\mathrm{PCO}_{2}$ of end-expiratory gas is measured during normal quiet breathing. This represents alveolar and arterial $\mathrm{PCO}_{2}$. A volume of gas is then equilibrated with mixed venous blood by rebreathing, and its $\mathrm{PCO}_{2}$ measured. The derived $\mathrm{A}-\mathrm{V}$ difference of $\mathrm{CO}_{2}$ tension is converted to $\mathrm{A}-\mathrm{V}$ difference of $\mathrm{CO}_{2}$ content. With the measurement of $\mathrm{CO}_{2}$ production, pulmonary blood flow can be calculated.

The lack of data concerning the cardiovascular adjustments to pregnancy in women with heart disease led the authors to make use of this method for determining cardiac output serially and at frequent intervals in a group of pregnant women with rheumatic heart disease.

Over 100 cardiac output determinations were made during pregnancy and the post partum period on 11 women with rheumatic heart disease. Control studies were made on normal non-pregnant subjects, and on pregnant women without heart disease.

The variability of this measurement in non-pregnant control subjects was less than \pm 10 per cent of the mean.
Comparable consistency of results was found between consecutive cardiac output determination in pregnant subjects, during the period of pregnancy when cardiac output is known to change only gradually.

In all pregnant subjects studied there was an increase in cardiac output reaching its peak during the third trimester, and decreasing before parturition. The mean increase was less in pregnant cardiacs than in pregnant normals.

It is concluded that, because of the reproducibility of results and ease of application, the method described is useful for making serial determinations of cardiac output.

\section{The Influence of Pitressin and Osmolar Excretion on} Urine Water Concentration in Normal Man. J. D. Crawford,* A. N. Cushman, A. Parisi, and M. L. TERry, Boston, Mass.

The effect of variations in solute outflow on the ratio of water to total osmotically active solute in urine (urine "water concentration") has been studied in normal water loaded human individuals during infusion of constant doses of Pitressin. Pitressin influence on urine water concentration is first observed at $0.07 \mathrm{mU}$ and is maximal at $0.75 \mathrm{mU}$ per m." per minute. When Pitressin is administered at rates less than $0.17 \mathrm{mU}$ per $\mathrm{m}^{2}$ per minute, the water concentration of urine progressively diminishes with increasing osmolar output. This relationship is reversed when Pitressin is infused at any given rate greater than $0.17 \mathrm{mU}$ per m.2 per minute. The relations between osmolar output and urine water concentration at each of the Pitressin doses studied conform to the general equation:

$$
\log \Delta \mathrm{K}=\mathrm{a}+\mathrm{b} \log \mathrm{X}
$$

where $\Delta \mathrm{K}$ is the difference between observed urine water concentration and the constant $\mathrm{K}$; $\mathrm{a}$ is the intercept constant; $b$, the slope constant and $X$, the output rate of osmotically active solute in urine. Analysis of data obtained in the absence of Pitressin, at doses which give maximal renal water conservation and at several intermediate dose levels, indicates that correlation coefficients have maximal and highly significant values when $K$ is equal to approximately 6.5 . No trend in values for $K$ is observed with changing Pitressin dose.

The observations confirm and extend those of DeWardener (Clin. Sc., 1955, 14, 715) and Wirz (Helvet. physiol. Acta, 1956, 14, 353) indicating that all urine whether concentrated or dilute, is elaborated from a hypotonic precursor. This precursor fluid appears to have a volume which varies in proportion with excreted solute but a constant water concentration of $6.5 \mathrm{ml}$. per mOsm. corresponding to the aforementioned value for $K$. In addition, the observations define normal responsiveness to Pitressin(1), permit clinical differentiation between "Pitressin resistance" and normal responsiveness of limited extent and have important implications with regard to fluid tolerance. 
A Study of the Factors Affecting the Equilibrium Between Human Erythrocytes and Human Plasma for Radioactive L-Thyroxine. K. R. CRISPELL, * JosePH Coleman, and Harry Hyer, Charlottesville, Va.

Thyroxine is bound in plasma primarily to a specific protein (T.B.G.) and secondarily to albumin. Previous in vitro studies have demonstrated that human erythrocytes will bind radioactive thyroxine. This binding is inhibited by the presence of plasma and to a lesser extent by human albumin. Radioactive thyroxine which is bound to erythrocytes can be removed by incubation with plasma. This suggests there is an equilibrium between erythrocytes and plasma for thyroxine. Studies by others using kidney and heart support this hypothesis.

Two milliliters of Evans Blue Dye ( 0.5 per cent) will decrease the uptake of thyroxine by erythrocytes from 75 to 37 per cent but does not inhibit the binding by plasma. Two milliliters of Trypan Blue does not inhibit the uptake of thyroxine by the erythrocytes but does inhibit binding by plasma. The presence of this dye increases the uptake by erythrocytes in the presence of plasma from 9 to 37 per cent. A five per cent solution of glucosamine gives results similar to those obtained with Trypan Blue. Trypan Blue, thyroxine, and glucosamine appear to compete for the same binding points on plasma. This is possibly due to a common side group which could act as a site for chelation with a metal and protein of the plasma. Evans Blue Dye does not contain this side group and does not interfere with the binding by plasma. However, it does interfere with the binding by erythrocytes suggesting that the mechanism of binding by the cell of thyroxine differs from that of the binding by plasma.

This in vivo system may offer a simple way of testing substances which alter the equilibrium between cell and plasma for thyroxine. The availability of thyroxine to the cell may depend on factors which shift the equilibrium in favor of the cell.

The Survival, Distribution and Apparent Interaction with Capillary Endothelium of Transfused Radiosulfate Labeled Platelets in the Rat. Eugene P. CRONKITE,* Victor P. Bond, James S. Robertson, and Donald E. Paglia, Upton, L. I., N. Y.

The thrombocytopenic purpura produced by whole body irradiation can be prevented by transfusion of fresh platelets (Cronkite and Brecher). In the present studies, the survival, distribution and fate of platelets transfused into the irradiated thrombopenic rat were studied using the radiosulfur platelet labeling method of Odell et al., and appropriate counting and high-resolution autoradiographic techniques. A potential mechanism of platelet hemostatic activity has been demonstrated. Historadioautographs of tissues of irradiated thrombopenic rats sacrificed one hour after transfusion of "homozygous" platelets in number sufficient to raise the circulating platelets levels from 5,000 to $1.5 \times 10^{6}$ per mm. ${ }^{2}$ strongly suggested that either intact platelets, or radiosulfur-labeled material from the platelets adhered to and lined the thrombopenic endo- thelium. In rats sacrificed four days after transfusion, the radiosulfur activity was found almost exclusively in the splenic macrophages. The survival times of platelets in the normal and irradiated rats were indistinguishable, approximately four days. Definitive interpretation of the adherence to capillary endothelium at 24 hours of the radiosulfur-labeled material involves mathematical considerations of the resolving power possible with $S^{2 *}$ beta particles $(0.165 \mathrm{Mev})$. It is our belief that the thrombopenic endothelium has selectively taken up the radiosulfur-labeled material of the platelet, or perhaps the intact platelet. Whether this means that platelets contribute some sulfur-rich macromolecular substance (presumably an acid mucopolysaccharide) that is essential for small vessel integrity, or whether platelets play a more secondary role by initiating fibrin formation at a submicroscopic level is a matter for conjecture and further investigation.

Investigation of the Human Small Intestine by Intraluminal Biopsy. WILliam H. CROSBY * and BeNJAMIN H. Sullivan, Washington, D. C.

An instrument has been developed for obtaining biopsy material from the small intestine of the unanesthetized human. It consists of a capsule 11 by $17 \mathrm{~mm}$. containing a rotating, spring-activated knife which is triggered by suction. The suction also acts to draw a specimen of mucosa through a port in the wall of the capsule before the knife is sprung. When a biopsy is to be taken the knife is "cocked" and the capsule is swallowed. It is held captive on a $2-\mathrm{mm}$. polyethylene catheter which also serves to transmit suction and to retrieve the capsule after the biopsy has been taken. Because the mucosa is sucked away from the muscularis when the biopsy is taken, the knife does not cut deeply into the bowel wall, and detectable bleeding has not been encountered.

Although the instrument was developed to obtain specimens of jejunal mucosa it is used to take biopsies at different levels from the esophagus to the ilium. Multiple capsules swallowed in tandem are used to obtain simultaneous specimens at different levels or to take successive specimens at the same level during the absorptive process.

A brief motion picture film will be shown to demonstrate the application of the biopsy capsule to problems of clinical investigation and diagnosis. Photomicrographs will also be presented to show normal and abnormal specimens of human intestinal mucosa.

\section{Corticosteroid Binding by a Plasma Alpha Globulin. \\ William H. Daughaday,* St. Louis, Mo.}

Dialysis equilibrium experiments with normal human plasma and increasing amounts of hydrocortisone labeled with hydrocortisone-4-C $C^{\mathbf{t}}$ have provided evidence for two independent corticosteroid binding systems. One has a high affinity but low capacity; and the other has a low affinity but high capacity. Earlier experiments have indicated that the low-affinity high-capacity binding protein in human plasma is albumin. 
The high-affinity, low-capacity binding system possesses a high degree of specificity for corticosteroids in that many slight structural alterations markedly decrease the ability of the derived steroid to displace hydrocortisone4-C $\mathrm{C}^{14}$ and corticosterone-4-C $\mathrm{C}^{14}$ from the binding protein.

Equilibrium continuous flow paper electrophoresis has been used to identify the high-affinity binding protein. Using a low concentration of corticosterone-4-C $C^{\text {th }}$ and careful preliminary equilibration with the electrophoretic buffer, migration of corticosterone-4-C $C^{\mathbf{4}}$ with an alpha globulin was clearly established. Moreover, dialysis equilibrium experiments with plasma protein fractions prepared by the Protein Foundation showed a high degree of hydrocortisone binding by fraction IV -4 , which contains alpha globulin.

Calculations of corticosteroid transport by normal human plasma with a hydrocortisone level of $14.2 \mu \mathrm{g} . / 100$ ml. show that $13.7 \mu \mathrm{g}$. was bound to alpha globulin, $0.4 \mu \mathrm{g}$. bound to albumin, and $0.2 \mu \mathrm{g}$. in free solution. With a plasma hydrocortisone level of $61 \mu \mathrm{g} . / 100 \mathrm{ml}$., only $16.6 \mu \mathrm{g}$. would be bound to alpha globulin, $26.9 \mu \mathrm{g}$. would be bound to albumin and $17.5 \mu \mathrm{g}$. would now be in free solution.

The plasma from six normal adults possessed comparable corticosteroid binding globulin activity. Notable decreases in corticosteroid binding globulin activity have been found in a preliminary survey of several diseases associated with abnormal plasma proteins, including cirrhosis, nephrosis and multiple myeloma. Increased corticosteroid binding globulin activity has not yet been encountered.

\section{Maintenance of Potassium Excretion Despite Reduction} of Glomerular Filtration During Sodium Diuresis. Douglas G. Davidson, Norman G. Levinsky, and Robert W. BerLiner,* Bethesda, Md.

It has been postulated that under physiologic conditions the filtered potassium is reabsorbed proximally and secreted potassium is the source of urinary potassium. The mechanism for potassium secretion which has been suggested is an ion exchange of potassium for sodium distal to the site of reabsorption. When the amount of sodium reaching the distal tubule is not a limiting factor in the exchange of potassium for sodium, the rate of potassium excretion should be independent of changes in the amount of potassium filtered.

To test this hypothesis the glomerular filtration rate was reduced in one kidney by an inflatable cuff around the renal artery. The contralateral kidney served as a control. Urine was collected separately from each kidney. Diuretics were given along with isotonic, sodium-containing solutions to assure that an adequate amount of sodium would reach the site of the postulated sodiumpotassium exchange.

With reduction of the inulin clearance of one kidney by up to $\mathbf{4 0}$ per cent the rate of excretion of potassium by that kidney remained equal to that of the control. Greater reductions of inulin clearance were associated with some decrease in potassium excretion relative to the control kidney.

The data strongly support the hypothesis that under these conditions the filtered potassium makes little or no contribution to that excreted in the urine and that the urinary potassium is derived in major part from secretion by exchange for sodium.

The data also indicate that moderate reduction of glomerular filtration by this technique reduces filtration in all of the nephrons but that decreases in excess of 40 per cent may result in a reduction of the number of functioning nephrons.

\section{Urinary Oxalate Excretion in Relation to Renal Stone Formation. Eleanor F. Dempsey, Boston, Mass. (Introduced by Anne P. Forbes).}

Approximately 64 per cent of renal stones contain significant amounts of calcium oxalate. Hypercalcuria apparently is a major cause of many of these stones; a concentrated urine due to dehydration may be a factor in others. The object of this study was to clarify the significance of oxalate excretion. Twenty-four-hour urinary oxalate (Dawson's method) has been measured in 28 urines from 20 normal subjects and in 120 urines from 77 patients with possible disorders of oxalate excretion while on normal mixed diets.

Thirty normal subjects and 31 patients with calcium oxalate renal stones excreted 15 to $45 \mathrm{mg}$. of urinary oxalate per day. Six of the stone patients had hypercalcuria; 19 had normal urinary calcium excretion. Three other patients with idiopathic hypercalcuria and calcium oxalate stones had urinary oxalate excretions of 50 to $80 \mathrm{mg}$. per day. A child with multiple recurrent calcium oxalate stones without hypercalcuria excreted 130 to $180 \mathrm{mg}$. per day. Hypercalcuria without stones was not associated with increased oxalate excretion in two patients with hyperparathyroidism, one with lung cancer without bone metastases, two with idiopathic hypercalcuria, and one with hypoparathyroidism on vitamin D. Two patients with sarcoidosis, hypercalcuria, and calcium oxalate stones excreted 60 to $110 \mathrm{mg}$. per day. Four patients with sarcoidosis, two of whom had hypercalcuria and one who had a calcium oxalate stone, excreted 17 to $39 \mathrm{mg}$. per day. Five of six patients with hepatic cirrhosis without renal stones excreted 70 to 80 mg. per day. In extended studies of three subjects on the metabolic ward, constant diets rich in oxalate increased the urinary oxalate excretion to two to four times the value observed on low oxalate diets. Forty grams of gelatin added to a constant diet doubled the urinary oxalate excretion.

Adrenal Steroids and Water Metabolism. JoSEPH F. Dingman and Rene H. Desporntes, Boston, Mass., and New Orleans, La. (Introduced by George E. Burch).

The effect of adrenal steroids on solute-free water clearance $\left(\mathrm{C}_{\mathrm{H}_{2} \mathrm{O}}\right)$ and the release of antidiuretic hormone 
$(\mathrm{ADH})$ in response to nicotine and hypertonic saline stimulation of the neurohypophysis was evaluated in 9 normal subjects and 2 patients with Addison's disease during constant water loading studies. Cortisone or hydrocortisone (50 to $100 \mathrm{mg}$.) given to normal subjects orally prior to hydration or intravenously during study augmented the rate of solute-free water diuresis and elevated the dose of nicotine required to stimulate $\mathrm{ADH}$ secretion. Intravenous prednisolone, intramuscular DCA and oral or intravenous fluorohydrocortisone had similar effects in 2 studies with each steroid. In 2 other studies with fluorohydrocortisone, $\mathrm{C}_{\mathrm{H}_{2} \mathrm{O}}$ was slightly depressed and nicotine antidiuresis prolonged or threshold stimulating dose diminished. Two Addisonians off cortisone therapy demonstrated delayed and subnormal diuretic responses to hydration and enhanced sensitivity to nicotine. Increased water diuresis and elevation of nicotine threshold were evident in one patient given $25 \mathrm{mg}$. cortisone orally prior to hydration and in both patients given $25 \mathrm{mg}$. prednisolone intravenously during study. The antidiuretic response to intravenous hypertonic saline and Pitressin was not altered by adrenal steroids. The increase in $\mathrm{C}_{\mathrm{H} 2 \mathrm{O}}$ and nicotine threshold was not correlated with variations in solute or sodium excretion. The observations suggest that extracellular hypotonicity induced by water administration does not completely suppress the neurohypophysis and that adrenal steroids increase excretion of solute-free water by additional inhibition of hypothalamic mechanisms controlling ADH secretion which may not be governed by osmoregulatory influences. In some instances, potent salt-retaining steroids may facilitate ADH release. The delayed diuresis of adrenal insufficiency may be attributed, in part, to persistent neurohypophyseal secretion unopposed by adrenal steroids.

On the Mechanism of Transmission of the Atrial Impulse to the Ventricle. Joseph R. DiPalma,* Philadelphia, $\mathrm{Pa}$.

The commonly observed clinical fact that alterations in $P-R$ interval in the ECG are always accompanied by changes in the contour of the $P$ wave was the background for this investigation. The chest was opened in twentyfive dogs under barbiturate anesthesia and electrodes inserted on the S-A node and in the right atrium in the region of the $\mathrm{A}-\mathrm{V}$ node. The electrode at the S-A node was recorded as an injury or monophasic potential; at the A-V node as a non-polarizable or diphasic potential. Recording was simultaneous by a twin direct writer and oscilloscope. Information derived from the records was the transmission time from S-A node to $A-V$ node, the relations of the contour of the monophasic to the diphasic action potential and the time sequence of the excitation of the A-V node to the action potentials.

It was found that normally the $A-V$ node fires at a predictable position on the contour of the monophasic action potential. This is always at the break in the recovery curve from slow to fast repolarization. Drugs such as epinephrine which shorten the position of this break in the curve consequently shorten the P-R interval. In contrast, stimulation of the vagus and acetylcholine which eliminate the break in the recovery curve lengthen the P-R interval by shifting the position of firing of the $\mathrm{A}-\mathrm{V}$ node to the bottom of the curve. In all experiments a change in the $P-R$ interval was always accompanied by a corresponding change in the contour of the atrial action potential. This was represented even in the imperfect rendition of the $P$ wave in the ECG limb leads. Actually the study indicated that the delay normally observed in atrial-ventricular transmission resides in the A-V node itself and is dependent on the peculiar excitable process of nodal tissue.

Drug Driving of the Heart in Man. Harold T. Dodge and H. V. Murdaugh, JR., Durham, N. C. (Introduced by H. O. Sieker).

Others have shown isopropylnorepinephrine increases the force of cardiac contraction in experimental animals. This is a study of the cardiovascular effects of isopropylnorepinephrine in man.

Studies were performed before and during intravenous infusion of isopropylnorepinephrine ( 1 to 2 gamma per minute) for periods as long as 50 minutes. In eight subjects, three normal and five with heart failure, right heart catheterization was performed with measurement of cardiac outputs, Fick method, and pulmonary arterial pressures.

During isopropylnorepinephrine infusion all subjects showed an increase in cardiac output (mean 37 and 63 per cent from mean basal cardiac indices of $3.40 \pm 0.44$ and $2.10 \pm 0.68$ in normal and heart failure groups, respectively) and a decrease in $\mathrm{A}-\mathrm{V}$ oxygen difference $(1.0 \pm 0.03$ and $3.0 \pm 1.63$ volumes per cent in the two respective groups). Oxygen consumption remained unchanged. Heart rate usually increased slightly, brachial arterial pulse pressure increased with little change of mean arterial pressure, peripheral vascular resistance decreased, and left ventricular work increased in all subjects. All but one of the patients with heart failure had an increase of left ventricular stroke volume (mean 30 per cent) and stroke work (mean 45 per cent). This was associated with no change or a decrease of pulmonary arterial diastolic pressure. Right-sided filling pressure decreased in two subjects where this was measured.

Renal clearances were performed in three subjects. Urine flow increased in all. G.F.R. remained unchanged in two subjects with normal control G.F.R. values. In the third subject with severe congestive heart failure G.F.R. increased from 30 to $139 \mathrm{ml}$. per minute.

These studies demonstrate isopropylnorepinephrine has a positive inotropic effect on normal and failing hearts. This occurs whether or not the patient is receiving digitalis, suggesting it increases cardiac contractility through a different mechanism than does digitalis. The cardiorenal responses differ from what is observed when the heart is driven by exercise. 
Plasma NEFA as an Index of Carbohydrate Utilization. Vincent P. Dole,* Edwin L. Bierman, and Thomas N. RoBerts, New York, N. Y.

In normal subjects the concentration of NEFA (nonesterified fatty acids) in plasma falls sharply after administration of glucose or insulin, increases slowly with fasting, and rises to exceptional levels after injection of epinephrine. The present studies suggest that these responses might provide a clinically useful index of changes in carbohydrate utilization. Since plasma NEFA has a half time of only a few minutes, the concentration of these fatty acids responds almost immediately to any variation of turnover rate.

Oral glucose or intravenous glucagon (as exogenous or endogenous glucose load, respectively) was given to normal and to diabetic subjects. With either kind of load the diabetics showed a delayed fall of NEFA, a subnormal amplitude of response and a slow return to baseline. Patients with diabetic acidosis and incipient coma showed a progressive rise in NEFA, preceding the onset of ketonuria, and little response to glucose. Treatment with insulin caused a prompt fall of NEFA, occurring several hours before the disappearance of ketonuria.

Although the NEFA response to glucose load seems to require insulin, the reduction probably is not a direct effect of this hormone since fructose, which can be metabolized independently of insulin, caused a prompt fall of NEFA. The pituitary hormones also appeared not to be primarily involved in the response since a sharp fall of NEFA was produced by feeding glucose to a hypophysectomized patient. Orinase $(2 \mathrm{gm}$.) injected intravenously into normal subjects or stable diabetics caused a fall of both glucose and NEFA. However, when given to juvenile diabetics with histories of repeated ketosis, orinase failed to reduce either glucose or NEFA, whereas insulin caused a prompt reduction of both.

The results to date suggest the hypothesis that utilization of carbohydrate inhibits release of NEFA from fat depots. In the unstable diabetic this mechanism seems to be faulty.

A Dual Vitamin $B_{12}$ Plasma Absorption Mechanism. Alfred Doscherholmen and Paul S. Hagen, Minneapolis, Minn. (Introduced by Edmund B. Flink).

Plasma vitamin $B_{1 s}$ absorption curves have been obtained by a radiometric technique in patients with pernicious anemia with and without intrinsic factor as well as in control subjects after the administration of oral test doses ranging from $0.56 \mu \mathrm{g}$. to $500 \mu \mathrm{g}$. radioactive vita$\min B_{12}$.

Patients with pernicious anemia were found to have no or negligible plasma absorption when oral test doses of $0.56 \mu \mathrm{g}$. were administered alone. When intrinsic factor concentrate was added, absorption curves were obtained similar to those found in control subjects given test doses in the same range. They were characterized by no plasma radioactivity during the first four hours of the test and a peak concentration in the 8 to 12 hour interval.
Oral test doses of 50 to $300 \mu \mathrm{g}$. vitamin $B_{12}$ given alone to patients with pernicious anemia resulted in completely different absorption curves. They were characterized by an early rise in plasma radioactivity and peak concentrations usually 3 to 6 hours after the test. This type of plasma absorption of vitamin $B_{12}$ appears to be independent of intrinsic factor. The addition of this factor abolished the early rise in plasma concentration and resulted in a peak concentration at 12 hours. Although intrinsic factor concentrate inhibited the plasma absorption not mediated through this factor, it enhanced hepatic uptake of radioactivity.

Control subjects given test doses of 200 to $500 \mu \mathrm{g}$. vitamin $B_{12}$ revealed a slightly diphasic plasma absorption curve with an early rise in the radioactivity. A test dose of $10 \mu \mathrm{g}$. gave a curve similar to those observed with test doses of $0.56 \mu \mathrm{g}$., while doses of 50 to $100 \mu \mathrm{g}$. revealed intermediate curves.

The dual vitamin $B_{12}$ plasma absorption mechanism lends support to the hypothesis of two distinct gastrointestinal modes for the absorption of this vitamin.

Comparative Analysis of Radioactive Strontium and Calcium Administered Simultaneously to Man. Elias C. Dow, William H. Baker, and John B. Stanbury,* Boston, Mass.

When calcium ${ }^{4 s}$ and strontium ${ }^{\mathrm{ss}}$ are administered intravenously to man, a larger fraction of the calcium ${ }^{45}$ is found in the bones. In dog and man, renal excretion of strontium $^{\text {ss }}$ is faster than calcium. It has not been clear whether differences in deposition of calcium and strontium in bone result from differential handling by bone or by kidney, or by both.

A single intravenous injection of strontium ${ }^{85}$ and calcium $^{2 s}$ was given to several patients with bone disease. For the succeeding three weeks or more, serum, urine, and feces were measured for content of calcium ${ }^{40}$, calcium $^{45}$, and strontium ${ }^{85}$. In all cases the urinary excretion of strontium ${ }^{85}$ was greater than the excretion of calcium $^{\text {ss, }}$, resulting in at least 30 per cent less body retention of strontium ${ }^{85}$ than calcium ${ }^{\mathrm{ss}}$ after 21 days. Urinary specific activities of strontium ${ }^{\mathrm{ss}}$ (per cent administered dose of strontium ${ }^{85}$ per gram of calcium ${ }^{20}$ ) were initially 3 to 8 times the urinary specific activities of calcium (per cent administered dose of calcium ${ }^{\mathrm{s}}$ per gram of calcium $^{20}$ ) and decreased exponentially. However, the specific activities of strontium ${ }^{86}$ in the serum were equal to those of calcium for one to five days, but then fell to significantly lower values. The specific activity of strontium $^{86}$ in the stools was higher than that of calcium ${ }^{45}$.

Simultaneous "pool sizes" (per cent of isotope retained in body per specific activity of isotope in the serum) were calculated for successive three-day periods for each isotope. In spite of increased renal and fecal excretion of strontium ${ }^{85}$, the "pool sizes" of strontium and calcium were virtually the same in each case studied.

The data indicate that renal excretion is a major factor controlling the amount of strontium ${ }^{85}$ available for 
deposition in bone, but bone does not discriminate between strontium $^{86}$ and calcium ${ }^{45}$.

The Production of a Hypernatremia and Hyperosmolarity in the Diabetes Insipidus Dog Infused with Ringer's Solution. Leonard S. Dreifus and Jeremiar StamLER,* Chicago, Ill.

This study was undertaken to assess the response of the unanesthetized diabetes insipidus (DI) dog to prolonged infusion of Ringer's solution ( 9 to $11 \mathrm{cc}$. per minute for several hours). In contrast to the ability of the intact animal to maintain constancy of plasma $\mathrm{Na}$ and total solute concentrations during infusion, DI dogs developed a progressive marked hypernatremia and hyperosmolarity. This alteration in plasma was a resultant of the abnormal renal $\mathrm{H}_{2} \mathrm{O}$-electrolyte exchanges in the $\mathrm{DI}$ dog. Thus, the DI animal exhibited normal rates of $\mathrm{Na}$ and total solute excretion (despite depressed RPF and GFR), but markedly elevated urinary flow rates. Hence, the DI animal elaborated a grossly hypotonic urine, in contrast to the approximately isotonic urine of the intact dog. These responses to prolonged Ringer's infusion were typical manifestations of the derangement in renal function in the DI animal. These findings may be applicable to man: 1. Diabetes insipidus may be differentially diagnosed by demonstrating hypernatremia in response to Ringer's infusion. (This test would obviate need for infusion of distressing hypertonic $\mathrm{NaCl}$ solutions.) 2 . Diabetes insipidus patients, as well as patients with transient DI due to intracranial disease or injury, should be approached very cautiously when saline infusions are contemplated; during such infusions, frequent sodium and/or total solute determinations are indicated to avoid production of hypernatremic and hyperosmolar states.

Effects of the Formic Acid Analogues of Thyroxine and Triiodothyronine on Cholesterol Metabolism. CHARLES

H. Duncan and Maurice M. Best, Louisville, Ky. (Introduced by J. Murray Kinsman).

Four thyroxine-like compounds have been investigated with regard to their relative effectiveness in preventing thiouracil-induced goitre and in effecting a reduction in serum and liver cholesterol levels. So studied were 1-triiodothyronine (T-3), 1-thyroxine ( $\mathrm{T}-4)$, triiodothyroformic acid (TFA-3), in which a carboxyl group has been substituted for the alanine side chain of triiodothyronine, and tetraiodothyroformic acid (TFA-4), the comparable formic acid analogue of thyroxine.

In the control group of 300 -gram male albino rats, the addition of 1 per cent cholesterol and 0.5 per cent thiouracil to their diet for 2 weeks resulted in a mean increase in thyroid weight from 6.5 to $18.9 \mathrm{mg}$. per $100 \mathrm{gm}$. body weight. The minimum daily dose per $100 \mathrm{gm}$. body weight of each of the four compounds which prevented thyroid enlargement was as follows: $\mathrm{T}-3,0.8 \mathrm{mcg}$.; $\mathrm{T}-4$, 3 mcg.; TFA-3, $900 \mathrm{mcg}$; and TFA-4, $3500 \mathrm{mcg}$.

The mean liver cholesterol in the control animals was $1162 \mathrm{mg}$. per $100 \mathrm{gm}$. liver. Each of the four compounds, administered in approximately the goitre-preventing dose, exerted some inhibitory effect on cholesterol accumulation in the liver. T-3, 0.7 mcg., resulted in liver cholesterol of $663 \mathrm{mg}$.; T-4, $3 \mathrm{mcg}$., in mean liver cholesterol of $744 \mathrm{mg}$.; TFA-3, $800 \mathrm{mcg}$., in mean liver cholesterol of $615 \mathrm{mg}$.; and TFA-4, $2000 \mathrm{mcg}$., mean liver cholesterol of $346 \mathrm{mg}$. All doses are expressed as mcg. per $1000 \mathrm{gm}$. body weight per day, and liver cholesterol as $\mathrm{mg}$. per $100 \mathrm{gm}$. liver. The disproportionately greater effect of TFA-4 on liver cholesterol was also demonstrated at other dosage levels. 1-Thyroxine, $6 \mathrm{mcg}$., twice the goitre-preventing dose, effected a reduction in liver cholesterol to $599 \mathrm{mg}$. per $100 \mathrm{gm}$. In contrast, TFA-4 at 4000 mcg., just above the goitre-preventing dose, resulted in liver cholesterol of only $268 \mathrm{mg}$. per $100 \mathrm{gm}$., essentially that of the rat fed neither cholesterol nor thiouracil.

TFA-4 has been administered to human subjects. In daily doses of $200 \mathrm{mg}$., it was found to be metabolically active as evidenced by increased rate of oxygen consumption and decrease in serum cholesterol.

Some Determinants of Urine Composition in Man: Separated Renal Functions in Patients with Unilateral and Bilateral Renal Diseases. HARrIET P. Dustan and Eugene F. Poutasse, Cleveland, O. (Introduced by A. C. Corcoran).

Glomerular filtration rate changes have been considered responsible for the small urine volume with low sodium concentration characterizing renal artery constriction in the dog and renal artery occlusive disease in man. To investigate this, separated renal functions were determined in thirteen hypertensive patients with unilateral or bilateral renal diseases.

Group I was composed of seven patients with nephrosclerosis; Group II, two with unilateral pyelonephritis; Group III, two with unilateral renal artery occlusive disease; Group IV, two with occlusive disease on one side and pyelonephritis or hydronephrosis on the other. Ureteral urines were collected during mannitol loading and Pitressin anti-diuresis; standard clearance studies were performed; water and sodium conservations were calculated.

In Group $I$, the differences in renal plasma flow (RPF), filtration rate (GFR) and filtration fraction (FF) between the two sides were as reported by Chasis and Redish. In six of the seven, urine osmolality ( $U_{\text {mosm }}$ ), water reabsorption ( $\mathrm{T}^{\mathrm{c}}{ }_{\mathrm{H}_{2} \mathrm{O}} / \mathrm{C}_{\mathrm{Mnn}}$ ), and per cent reabsorption of filtered sodium load ( $\% \mathrm{Na}$ Reab.) were similar. Small GFR differences, in four, were accompanied by parallel differences in sodium concentration $\left(U_{\mathrm{N}_{2}}\right)$. In Group II, there were marked disparities in RPF and GFR with corresponding changes in urine volume (V), but $\mathrm{U}_{\mathrm{mosm}}, \mathrm{T}^{\mathbf{c}}{ }_{\mathrm{Hz}} / \mathrm{C}_{\mathrm{Man}}$, and $\% \mathrm{Na}$ Reab. were identical. In Group III, RPF, GFR, FF and V were all decreased on the involved side; in one patient, these changes were associated with markedly lowered $\mathrm{U}_{\mathrm{Na}}$, increased \% $\mathrm{Na}$ Reab., $\mathrm{U}_{\mathrm{mOsm}}$, and $\mathrm{T}^{\mathrm{c}} \mathrm{H}_{2} \mathrm{O} / \mathrm{C}_{\mathrm{Man}}$; in the other $\mathrm{T}^{\mathrm{c}}{ }_{\mathrm{H}_{2} \mathrm{O}} / \mathrm{C}_{\mathrm{Man}}$ was, perhaps, significantly increased but $U_{\text {mosm }}, U_{\mathrm{Na}}$, and \% $\mathrm{Na}$ Reab. were the same on both sides. In Group IV, the kidneys with the artery lesions had higher RPF and 
GFR than their mates, with corresponding but not impressive changes in $V$, yet, in spite of this, $U_{m 0 s m}$, $\mathrm{T}_{\mathrm{H}_{2} \mathrm{O}} / \mathrm{C}_{\mathrm{Man}}$, and \% $\mathrm{Na}$ Reab. were increased.

The data suggest that GFR is not, alone, responsible for urine composition in unilateral renal disease.

The Effect of Exposure of Erythrocytes from Acid Citrate Dextrose $(A C D)$ Whole Blood to 5 Grams Per Cent Dextrose and 0.225 Grams Per Cent Sodium Chloride on RBC Size, Potassium Concentration and Content, Glucose Content, and In Vivo Survival in Man. Franklin G. Ebaugh, JR., Joseph R. Bove, and Richard S. Stemler, Hanover, N. H. (Introduced by S. Marsh Tenney).

Exposure of erythrocytes (1 part whole ACD blood to 2 parts dextrose solution) to 5 per cent dextrose and $0.225 \mathrm{gm}$. per cent $\mathrm{NaCl}$ for 3 hours results in $138 \pm 5$ (134 to 177) per cent increase in hematocrit, $1100 \pm 270$ (690 to 1660) per cent increase in glucose, and a decrease in potassium concentration to $58 \pm 5.7$ (50 to 63 ) per cent of normal, but no change in $\mathrm{K}$ content per RBC. Three minutes after replacement of the dextrose solution with autogenous plasma the hematocrit was $149 \pm 14$ per cent that of the ACD whole blood. The hematocrit was corrected for hemolysis occurring in 10/20 of the observations during addition of plasma. After one hour in plasma the hematocrit, RBC glucose, and $\mathrm{K}$ concentration returned to normal. The in vivo survival 10 minutes after transfusion of $\mathrm{Cr}^{\text {sit }}$ labeled $\mathrm{RBC}$ previously exposed to dextrose solution for 3 hours ranged from 5 to 127 per cent the transfused amount and showed no correlation with the hematocrit increase, or the RBC glucose concentration, but did show a significant inverse correlation $(r=-0.565, n=14, p<.05)$ with the per cent increase in hematocrit after subsequent 3-minute equilibration with plasma. The in vivo survival of the same RBC in the same recipient was increased $14 \pm 19$ per cent $(p<.05)$ after equilibration with plasma for one hour. The survival of one part ACD blood and 19 parts of dextrose solution, in contrast to the $1 / 3$ mixture, showed a consistent relationship between survival and time exposed to dextrose solution, the results being $50,20,15$ and 8 per cent survival after $30,60,90$, and 120 minutes, respectively. The above data indicate that an increase above $170 \pm 10$ per cent normal RBC size under the above conditions is incompatible with normal RBC in vivo survival, and that exposure to the dextrose solution exerts a slight but significant deleterious effect on RBC survival apart from $R B C$ volume change.

The Pressure-Volume Characteristics of Human Forearm Veins During Epinephrine Infusion. JонN W. Ecrstein and Wirliam K. Hammion, Iowa City, Ia. (Introduced by James W. Culbertson).

Six normal men were studied in the supine position with a plethysmograph on the left forearm, a pressure needle in the right antecubital vein, and an infusion needle in a foot vein. Forearm venous distensibility (VD) was measured plethysmographically (Litter, Wood, Wilkins). Pressure-volume curves obtained by this method originate at the volume existing at an effective venous pressure (EVP) of $1 \mathrm{~mm}$. $\mathrm{Hg}$. Curves are formed by plotting volume changes against increments in EVP from 1 to $31 \mathrm{~mm}$. Hg. The point on each curve corresponding to the local venous pressure (LVP) measured in the unencumbered (right) forearm indicates local venous volume (LVV). Observations on VD and LVP were made before, during and after epinephrine infusion.

During control periods in 6 experiments VD for an EVP of $30 \mathrm{~mm}$. $\mathrm{Hg}$ averaged $4.1 \mathrm{cc}$. per $100 \mathrm{cc}$. of forearm tissue and ranged from 3.0 to 4.5 . When epinephrine was infused at $15.0 \mathrm{mcg}$. per $\mathrm{min}$. the average value fell to $2.6 \mathrm{cc}$. per $100 \mathrm{cc}$. and the range was 1.9 to 3.3. LVP rose during this infusion with increases which averaged $2.9 \mathrm{~mm}$. $\mathrm{Hg}$ and ranged from 1.5 to 5.5 . During control periods LVV averaged $1.9 \mathrm{cc}$. per $100 \mathrm{cc}$. and ranged from 1.5 to 2.5 ; with epinephrine these values fell to levels averaging $1.4 \mathrm{cc}$. per $100 \mathrm{cc}$. and ranging from 1.0 to 1.9 .

In 8 additional experiments epinephrine infusions of 7.5 or $10.0 \mathrm{mcg}$. per min. regularly caused reductions in VD and increases in LVP while inconsistent small changes occurred in LVV.

Under the conditions of these 14 experiments epinephrine infusion uniformly caused venous pressure to rise and venous distensibility to decrease. At an infusion rate of $15.0 \mathrm{mcg}$. per min. the net effect in each case was a shift of blood out of the forearm veins.

The Relationships Among Serum Sodium Concentration, Serum Osmolarity and Body Composition in Man. I. S. Edelman,* J. Letbman, M. P. O'Meara, and L. W. Birkenfeid, San Francisco, Calif.

A quantitative relationship between electrolyte content of the body and serum sodium concentration has not been established in previous studies, nor is such a relationship predictable because of uncertainties with respect to osmotic equilibrium across cell membranes and possible mobilization of bone sodium in pathological states. The observations to be presented indicate that serum sodium concentration $\left(\mathrm{Na}_{3}\right)$ and serum osmolarity $\eta_{0}$ are linear functions of the ratio of exchangeable body sodium $\left(\mathrm{Na}_{0}\right)$ plus exchangeable body potassium ( $\mathrm{Ke}$ ) to total body water (TBW). This ratio $\left[\frac{\mathrm{Na} a_{0}+\mathrm{Ke}}{\mathrm{TBW}}\right]$ is a measure of the proportion of osmotically active cation to total body water.

Measurements of $\eta_{0}$ by freezing point depression and simultaneous $\mathrm{Na}$, have been performed in 69 patients. $\pi_{0}$ is a linear function of $\mathrm{Na}_{\text {s }}$ when appropriate corrections are made for serum NPN and glucose with an S.D. $\Pi_{\circ} / \mathrm{Na}$, of $\pm 5 \mathrm{mOsm}$. $\mathrm{Na}_{0}, \mathrm{Ke}$ and $\mathrm{TBW}$ were measured in 66 patients by isotope dilution. Thirty-six of these subjects were edematous because of cardiac, hepatic or renal disease. The Na, varied from 110 to $155 \mathrm{mEq}$. per $\mathrm{L}$. in these subjects. Neither $\mathrm{Na}$, nor $\prod_{0}$ showed a significant correlation with $\mathrm{Na}, \mathrm{Ke}, \mathrm{TBW}, \mathrm{Na} / \mathrm{TBW}$ or 
$\mathrm{Ke} / \mathrm{TBW}$. Both $\mathrm{Na}$, and $\mathrm{T}_{\mathrm{s}}$ showed a highly significant correlation with $\left[\frac{\mathrm{Na} \mathrm{a}_{0}+\mathrm{Ke}}{\mathrm{TBW}}\right]$. The covariance of $\mathrm{Na}$. with respect to this ratio was only $\pm 6 \mathrm{mEq}$. per $\mathrm{L}$.

These results support the following conclusions:

a) The primary determinants of serum sodium concentration are body sodium, potassium and water contents and provide the basis for a classification system for hypo- and hyper-natremias,

b) Extracellular and intracellular fluids are in osmotic equilibrium.

Metabolic Interrelations of Calcium and Magnesium in Patients with and without Osteolytic Disease. LEONARD P. Eliel,* Winliam O. Smith, and Josephine HawRYLKo, Oklahoma City, Okla.

Tremors, convulsions and hypomagnesemia of 0.9 to $1.1 \mathrm{mEq}$. per L. (normal mean $1.60 \mathrm{mEq}$. per L.) were observed in three patients with osteolytic disease in association with episodes of hypercalciuria, hypercalcemia, and renal impairment. Urine excretion of magnesium varied directly with urine calcium and in twenty-fold greater amount than could be accounted for on the basis of bone destruction alone. Balance data revealed intracellular and extracellular magnesium depletion. When a hypercalciuria of kelated calcium was produced, in one of the patients, with intravenous EDTA, however, no increase occurred in urinary magnesium.

Two patients without osteolytic disease and two with osteolysis, but with normal renal function, were studied by daily intravenous calcium loading while on metabolic balance regimes. The urine magnesium decreased to 20 to 33 per cent of the control values while urine calcium increased by 3 to 70 fold. However, the serum magnesium declined to 36 to 75 per cent of control values; levels as low as $0.66 \mathrm{mEq}$. per L. were noted. Stool magnesium showed little change. A diuresis of sodium, chloride and water occurred in all patients. Balance calculations revealed movement of magnesium into the cells. None of these patients developed symptoms of magnesium deficiency. Similar changes in magnesium did not occur with infusions of an equivalent amount of sodium.

It appears that the normal metabolic response to intravenous calcium loading is an intracellular shift of magnesium with a resultant decrease in its extracellular concentration and renal excretion. Patients with osteolytic disease and renal impairment show increased renal loss and extracellular depletion of magnesium during hypercalciuria of ionized calcium, presumably due to tubular damage.

Collateral Sensitivity to Methotrexate in 6-Mercaptopurine-responsive Adult Acute Leukemics. Rose RUTH Ellison, New York, N. Y. (Introduced by Joseph H. Burchenal).

Certain antimetabolites may produce temporary remissions of acute leukemia. These effects apparently result from blocking nucleic acid biosynthesis at some point. Bacterial and in vitro studies have indicated that the folic acid antagonists and 6-mercaptopurine (6MP) act differently, the former inhibiting de novo synthesis of purines and pyrimidines and the latter affecting incorporation of purines into nucleic acid.

The folic acid antagonists produce temporary remissions in 35 to 50 per cent of children but are comparatively ineffective in adults (3 partial remissions and 1 clinical improvement in 38 cases). The data to be reported concern 29 adults with acute leukemia who initially responded to $6 \mathrm{MP}$ but developed resistance to $6 \mathrm{MP}$ and relapsed. They were then treated with the folic acid antagonist, 4-amino- $\mathrm{N}^{10}$-methyl pteroylglutamic acid (Methotrexate). Nine of the 29 showed some degree of response. Three developed complete hematologic and clinical remissions, 3 showed partial marrow but good clinical and peripheral blood effects, and 3 were clinically benefited with some improvement in the peripheral blood picture. This is an incidence of 31 per cent, substantially higher than the 11 per cent observed with Methotrexate alone.

The mechanisms responsible for the initial resistance of some leukemias to therapy, and for the development of resistance in the initially responsive are unknown. The occurrence of apparently increased sensitivity of the leukemic process to Methotrexate in the patients reported parallels findings by others in 6MP-resistant strains of both $L$. casei and $L 1210$ mouse leukemia. In $L$. casei the increased sensitivity of the 6MP-resistant strain to Methotrexate is associated with an increased requirement for folic acid. The clinical data suggest that either the 6MP-resistant strains of leukemic cells which evolved have pathways for nucleic acid synthesis qualitatively different from those previously utilized, or a Methotrexate-responsive group has been selected out by virtue of its also being responsive to 6MP.

Observations on the Action of Combined GlucagonInsulin Infusions in Diabetic Patients. HaroLd ElRICK, Y. Arai, and V. Yearwood-Drayton, Denver, Colo. (Introduced by Gordon Meiklejohn).

Studies from this laboratory have shown that in nondiabetic patients glucagon and insulin together augment peripheral glucose utilization more than either hormone alone. Furthermore, combined infusions resulted in neutralization of the opposing action of the two agents on arterial glucose levels.

The purpose of this paper is to report on 39 glucagoninsulin experiments in 33 diabetic patients. Under standardized conditions, after an 11-hour fast and 25 hours after the last dose of insulin, glucose (142 to $185 \mathrm{mg}$. per $\min$.) and insulin (3.3 to $6.5 \gamma$ per min.) were administered by constant intravenous infusion. Crystalline glucagon (1.1 to $5.1 \gamma$ per min.) was added 55 or 70 minutes following the start of the infusion. Arteriovenous samples (16 pairs) were obtained throughout a two and one-half-hour test period in 16 patients. A-V/A values (arteriovenous glucose difference/arterial glucose level: previously shown to be an adequate index of peripheral glucose utilization) were computed for each pair 
of samples. In 23 experiments arterial glucose samples alone were obtained.

Comparison of the control (insulin) with glucagon (glucagon + insulin) periods revealed a significant ( $P$ $=0.02$ ) increase in $\mathrm{A}-\mathrm{V} / \mathrm{A}$ values following glucagon administration in 11 ( 69 per cent) of the 16 patients; in the remaining patients no significant change occurred.

All experiments (39) were analyzed with regard to the relationship between glucagon/insulin ratio and arterial glucose levels. In 13 (mean ratio, 0.47) a neutralizing effect of the opposing action of the two agents on arterial glucose levels was obtained; in 24 (mean ratio, $0.76)$ hyperglycemia resulted; in 2 a biphasic reaction (ratio, 0.74) and blood sugar fall (ratio, 0.29) occurred.

This study indicates that a glucagon-insulin combination can produce greater enhancement of peripheral glucose utilization than insulin alone, as well as a stabilizing effect on arterial glucose levels. Such a combination, therefore, may prove useful in the management of some diabetic patients.

\section{Starch Gel Electrophoresis of Serum Proteins and Uri-} nary Proteins from Patients with Multiple Myeloma. Ralph L. Engle, Jr., KenNeth R. Woods, and James H. Pert, New York, N. Y. (Introduced by Paul Reznikoff).

Because potato starch gel electrophoresis (Smithies) has high resolving power, it was used to study the serum protein and urinary protein patterns of 27 patients with multiple myeloma. Twenty urine samples containing Bence-Jones proteins and 14 sera were examined. Some urine samples were first concentrated by dialysis against polyvinylpyrrolidine. In other instances the urinary protein was precipitated by $2 / 3$ saturation with ammonium sulfate, dialyzed, lyophilized, and applied to the gel as a 2 per cent solution.

Serum electrophoresis by this method gave more detailed and more distinct patterns than could be obtained by paper electrophoresis. The gamma-globulin was markedly decreased in all sera. Seven sera had single protein spikes in the gamma-globulin region; two sera had single spikes between the origin and slow-alpha globulin. One serum showed marked elevation of slowalpha ${ }_{2}$-globulin; another, elevation of beta-globulin. Three sera deficient in gamma-globulin had no protein spike. One of these contained $3 \mathrm{gm}$. per cent macroglobulin ( $s=$ about $19 \mathrm{~S}$ ) which migrated as a gammaglobulin upon paper electrophoresis. With gel electrophoresis this macroglobulin did not migrate. All sera showed decreases in albumin and many demonstrated complex abnormalities in other components.

Analysis of the urinary proteins revealed that 6 samples contained 3 to 4 components, and 10 contained 2 components, all in high relative concentration, usually migrating close together. Four samples contained single components. This contrasted with the single components found in most instances by paper electrophoresis. Traces of albumin were frequently observed. One specimen of urinary protein for which no indication of heterogeneity could be obtained by paper electrophoresis, boundary electrophoresis at three hydrogen ion concentrations, ultracentrifugation, and immunologic studies by Dr. L. Korngold (Ouchterlony) was clearly shown by gel electrophoresis to contain two closely associated fractions (43 per cent and 52 per cent, respectively).

By means of starch gel electrophoresis new information has been obtained on the serum proteins in multiple myeloma and on the heterogeneity of Bence-Jones proteins.

The Effect of Exclusion of the Erythematous Palm Upon

Venous Ammonia in Hepatic Cirrhosis. Curtis J. Fisher, Robert Eich, and William W. Faloon, Syracuse, N. Y. (Introduced by Eugene L. Lozner).

The association of palmar erythema with arteriovenous shunting of blood has been demonstrated in patients with hepatic cirrhosis. This observation may be of significance in studies of tissue metabolism as measured by arteriovenous differences. Arterial ammonia concentrations have been demonstrated to be appreciably greater than venous concentrations, suggesting the concept of peripheral utilization of ammonia, or as demonstrated by venous catheterization and in vitro studies that oxygenation may increase the "apparent" ammonia concentration. In an attempt to determine the effect of palmar erythema on venous ammonia, studies of arterial and venous ammonia and oxygen concentrations in 14 patients with liver disease, 9 with marked palmar erythema and 5 with minimal or no erythema, have been performed. In both groups of patients, studies were done before and during exclusion of the palm by a cuff inflated above systolic pressure.

Before exclusion no significant difference in the arteriovenous ammonia difference was observed between patients with erythema and those with none. Arteriovenous oxygen difference was less in patients with erythema. In contrast, with exclusion of the palm all patients with erythema showed a significant decrease in venous ammonia, averaging 45 per cent (range 27 to 75 per cent) of the control values. Thus a marked increase in arteriovenous difference in ammonia and oxygen occurred. Following removal of the cuff, these values returned to pre-exclusion levels. In patients with minimal or no erythema, exclusion had no effect upon venous ammonia or oxygen concentrations.

The results indicate that the presence of palmar erythema significantly alters the venous ammonia concentration. It is apparent that palmar erythema may similarly produce an error in measurement of other metabolites when peripheral arteriovenous techniques are used.

The Clearance Rate of $I^{181}$ Labelled Streptokinase from the Circulation of Man. Anthony P. Fletcher and Alan J. Johnson,* St. Louis, Mo., and New York, N. Y.

The fibrinolytic state induced in man by the intravenous administration of streptokinase offers a possible approach to the treatment of thromboembolic disease. 
Investigation of this phenomenon has been difficult in the absence of a method for the measurement of plasma streptokinase levels. Streptokinase of high purity and specific activity was labelled with $I^{181}$ by the method of Hughes et al. There was no loss of activity during the labelling process and the integrity of the bond was maintained during electrophoresis.

The dose of streptokinase required to produce fibrinolysis in patients can be predicted by in vitro testing of the serum inhibitory factors. The dose so calculated is termed the predicted dose and a comparison of these values with the results of streptokinase infusion into man has shown that its accuracy is \pm 15 per cent. Probit analysis showed that the predicted dose values in a sample of the population were distributed as a log normal variable.

Labelled streptokinase was cleared from the circulation at an exponential rate. The mean 50 per cent plasma clearance rate was extremely rapid, 18 min. (range, 5 to $35 \mathrm{~min}$.) if a smaller dose than the predicted was infused, but slower, $81 \mathrm{~min}$. (range, 58 to $110 \mathrm{~min}$.) if the clearance determination was made after streptokinase equivalent to the predicted dose had been injected. These two clearance rates were significantly different $(p<0.001)$. Furthermore, there was evidence of rapid streptokinase breakdown as evinced by the early appearance of free $I^{2 a 1}$ in the plasma and its excretion into the urine. Data are also to be presented on the levels of plasma streptokinase attained during continuous streptokinase infusion and on the accompanying changes in the components of the plasminogen-plasmin system.

The results indicate that the plasma streptokinase level is a crucial determinant in the fibrinolytic effect exhibited by plasma, after streptokinase infusion.

\section{Systolic-Diastolic Pressure Relationships in the Isolated}

Beating Heart. Noble O. Fowler and Walter L. BLoom,* Atlanta, Ga.

Bloom and Ferris observed in the left ventricle of the excised submerged beating rat heart positive pressure during systole, and negative pressure during diastolic filling by suction.

In the present study, systolic-diastolic pressure relationships were observed in 33 excised beating rat hearts under three conditions: (1) spontaneous deterioration; (2) ventricular alternans; (3) during epinephrine or 1-nor-epinephrine effect. Left intraventricular pressures were measured with reference to the hydrostatic pressure existing at the same level below the surface of the liquid in which the heart was submerged.

Results: Initially, peak systolic pressures were from 11 to $84 \mathrm{~mm}$. water and negative diastolic pressures were from 2 to $24 \mathrm{~mm}$. water.

In each of six hearts, regressions showed significant relation between peak systolic and diastolic nadir pressures during spontaneous weakening of the heart beat, $\mathrm{p}<0.001$.

With 1-nor-epinephrine or epinephrine, peak systolic and negative diastolic nadir pressures increased. A re- gression of change in diastolic pressure upon change in systolic pressure was significant, $\mathrm{p}<0.01$.

Usually, ventricular alternans was associated with less negative diastolic pressure following each weak systolic beat. Thus strong systolic beats followed less negative diastolic pressures. One heart showed $25 \mathrm{~mm}$. water systolic pressure and negative diastolic pressure of $8 \mathrm{~mm}$. during strong beats, and systolic pressure of $5 \mathrm{~mm}$. water with diastolic pressure of minus $1 \mathrm{~mm}$. during alternate weak contractions.

The results indicated significant association between positive systolic and negative diastolic pressure in the excised beating heart under three conditions: (1) spontaneous decay; (2) increase in contractile force produced by sympathomimetic drugs; (3) ventricular alternans. It is suggested that diastolic force is related to systolic force. The results are consistent with the concept that the force of diastole is due to a release of energy stored in systole and may not be associated with a chemical release of energy.

\section{Studies Using Glucose and Pentoses to Determine the Mechanism of the Hypoglycemic Response to Sulfonyl- ureas. Thomas F. Frawley and Stanton Segal,} Bethesda, Md. (Introduced by Richard T. Beebe).

Tolbutamide was given in amounts and by a route designed to produce a depression in blood sugar quantitatively similar to that obtained after insulin in the same individual. Following the rapid intravenous administration of sodium tolbutamide ( $18 \mathrm{mg}$. to $40 \mathrm{mg}$. per $\mathrm{Kg}$.) or insulin ( 0.1 unit per $\mathrm{Kg}$.) in two to three minutes an approximately 50 per cent decrease in blood glucose concentration was seen in thirty minutes. As with insulin the onset of the hypoglycemia after tolbutamide was immediate and accompanied by a depression in plasma phosphate. However, the tolbutamide-induced hypoglycemia was unaccompanied by the rise in blood pyruvate which occurs after insulin. When intravenous tolbutamide was substituted for insulin in the insulin-glucose tolerance test the hyperglycemic response following the hypoglycemia was suppressed, but not abolished, suggesting impairment of hepatic glycogenolysis. The glucose disappearance rate constant from blood after an acutely administered intravenous load of glucose $(0.3 \mathrm{gm}$. per $\mathrm{Kg}$.) was increased after intravenous tolbutamide or insulin. In one of the individuals studied a normal disappearance rate constant of 1.8 per cent per min. was increased to 4.0 per cent per min. with tolbutamide and to 7.2 per cent per min. with insulin, thus indicating that the tolbutamide effect on glucose disappearance closely parallels that of insulin. Twenty-gram quantities of $\mathrm{D}$-xylose and $\mathrm{L}$-arabinose were infused rapidly and the rate of disappearance from blood was compared after intravenous insulin and intravenous tolbutamide. Insulin accelerated the disappearance rate of both pentoses, decreasing the biological half-time to approximately one-third. Tolbutamide did not affect either pentose. The pentose observations do not suggest that tolbutamide enhances the release of and/or peripheral action of insulin. The com- 
bined studies on glucose and pentose disposition suggest that tolbutamide's hypoglycemic effects are primarily hepatic. This might be brought about by altering hepatic responsiveness to endogenous insulin.

Metabolism of Albumin-Bound Labeled Fatty Acids in Man. Donald S. Fredrickson and Robert S. Gordon, JR., Bethesda, Md. (Introduced by Robert P. Grant).

Previous studies indicate that the albumin-bound plasma unesterified fatty acids (UFA) may represent the major form in which fat is transported to tissues for oxidation. This hypothesis requires that plasma UFA turnover occur rapidly. The plasma turnover rate in man has been investigated by injecting intravenously $\mathrm{C}^{14}$-carboxyl-labeled fatty acids bound to albumin and measuring the disappearance of radioactivity from plasma and its appearance in expired $\mathrm{CO}_{2}$. Removal of radioactive UFA from the plasma followed a complex curve but followed first-order kinetics for 4 to 10 minutes after intravascular mixing. The half-time of disappearance during this phase varied from 1 to 3 minutes. Plasma turnover rates calculated by dividing the UFA concentration by the turnover time obtained from the early phase of the disappearance curve varied from $0.06 \mathrm{mEq}$. per liter per minute in subjects pre-fed carbohydrate to $0.5 \mathrm{mEq}$. per liter per minute in normals fasted 42 hours and in uncontrolled diabetics. Approximately 10 per cent of administered palmitate-1- $\mathrm{C}^{16}$ radioactivity was recovered as $\mathrm{C}^{14} \mathrm{O}_{2}$ in one hour in the fasted subjects, less in those fed carbohydrate. The peak specific activity occurred at 25 minutes. In two subjects, linoleate-1-C ${ }^{16}$ radioactivity appeared more slowly. The preliminary studies indicate that: 1) UFA molecules leave the plasma rapidly despite very tight binding to albumin; 2) the turnover of plasma UFA appears to be closely related to the contribution of fat to the total metabolic pool; 3) plasma UFA does not mix with a pool as great as the total depot fat prior to oxidation, although the origin of plasma UFA in fasting subjects appears to be the adipose tissue.

Clinical Application of Lupus Serum-Nucleoprotein Reaction Using the Fluorescent Antibody Technique. George J. Friou, West Haven, Conn. (Introduced by S. C. Finch).

Use of the fluorescent antibody technique has revealed that a factor in lupus serum adheres to nuclei in sections of animal tissues. More recently, this observation has been extended to normal human tissues.

Nucleohistone (TNH) was prepared from calf thymus and tested for participation in this reaction. It was found that sera which caused localization of fluorescent anti-human globulin on nuclei also caused it to adhere to spots of TNH on glass slides. Sera not causing nuclear localization were also negative with TNH. Additional evidence of the identity of the reactions was obtained by parallel tests against nuclei and $\mathrm{TNH}$ spots after absorption of positive sera with precipitated TNH.

Reproducible titers could be obtained by testing dilutions of serum against TNH spots. A large number of sera from patients with lupus and other diseases were tested by this method. Results indicate presence of the factor to be characteristic of lupus. Reactions at low dilution have also been observed with several sera from among the groups of patients with rheumatoid arthritis and allergic reactions to drugs, but not in other diseases.

Incomplete information indicates the probable identity of the serum factor with the factor responsible for L.E. cell formation. Because of the sensitivity of this method, and the semi-quantitative results that can be obtained, it should be applicable to study of many unanswered questions regarding lupus.

Effect of Acetylcholine in the Pulmonary Circulation of Man. H. W. Fritts, JR., P. Harris, R. H. Clauss, and A. Cournand, New York, N. Y. (Introduced by D. W. Richards).

One of us (P. H.) has observed that a single injection of acetylcholine can lower the pulmonary arterial pressure in patients with a moderate degree of pulmonary hypertension. Since the effect was not evident in normal subjects, it was suggested that the response to the drug depends on the pre-existing vascular tone. This hypothesis was based on two considerations: 1) the fact that in patients with pulmonary hypertension the muscular coats of the small vessels are hypertrophied, and 2) the assumption that this hypertrophy and an increased tone go hand in hand. In the present study, the hypothesis has been tested by using hypoxia to raise the tone of the pulmonary vessels of 8 normal subjects. Acetylcholine was infused into the main pulmonary artery at the rate of $0.5 \mathrm{mgm}$. per min. While the subjects breathed 21 per cent oxygen, the drug consistently lowered the systolic pressure in the pulmonary artery by from 1 to $7 \mathrm{~mm}$. Hg. The mean pressure was also lowered, and the diastolic pressure inconstantly reduced. No change was apparent in the pulmonary wedge pressure, the systemic blood pressure, the heart rate, or the cardiac output. After hypoxia had produced pulmonary hypertension, the fall in pressure with acetylcholine was more pronounced, despite the fact that during the infusion the cardiac output frequently increased. The mechanism of the effect is not known, although the same response was observed in one patient who had had all of the sympathetic ganglia removed. These results bear on the problem of vasomotricity in the lungs, and suggest that under certain conditions the pulmonary vessels have the capacity for intrinsic changes in tone.

Mechanism of the Glucosuria Produced by the Administration of Steroids with Glucocorticoid Activity. E. Rudolf Froesch, Albert I. Winegrad, and Albert E. Renold, Boston, Mass. (Introduced by George W. Thorn).

The mechanism of the glucosuria produced by adrenal corticoids has been investigated in four healthy young males and in two patients with renal glucosuria. Each subject served as his own control. In the normal subjects glucose titration and renal clearance studies were 
carried out on control days as well as 12 and 90 hours after instituting prednisone administration by mouth. Blood and urine glucose were measured by a specific enzymatic method and inulin determinations were carried out after enzymatic removal of interfering glucose. Each study consisted of at least ten consecutive periods. In all subjects prednisone administration was associated with increased blood glucose levels and an increased rate of glomerular filtration (inulin). These two factors accounted for the increased glucosuria. In no instance was TmG affected significantly nor was increased glucose excretion noted at glucose loads below $\mathrm{TmG}$. The two patients with renal glucosuria were kept fasting for 16 hours prior to hydrocortisone administration and throughout the study. Hydrocortisone was administered intravenously over ten hours. Blood glucose increased markedly in both subjects and urinary glucose excretion increased from 10 to $36 \mathrm{mg}$. per minute and from 5 to 25 mg. per minute, respectively. Again the increase in blood glucose and the increased glomerular filtration rate accounted for the increased urinary glucose excretion. Tubular glucose reabsorption was not significantly altered. There was a lag of 3 to 4 hours between the beginning of intravenous hydrocortisone administration and the onset of either the blood glucose or the glomerular filtration rate response. In two patients with Cushing's Syndrome and glucosuria TmG was measured and found to be within normal limits. It is concluded from these studies that the glucosuria produced by the administration of certain adrenal corticoids results from an increase in the filtered glucose load, not from decreased tubular glucose reabsorption.

Relation of Acquired Hemolytic Anemia to Macroglobulins. Hugh Fudenberg and Henry G. Kunkel,* New York, N. Y.

The sera of five patients with the syndrome of high titer cold agglutinins, paroxysmal cold hemoglobinuria, and chronic hemolytic anemia were studied by (starch) zone electrophoresis and analytical and (density gradient) zone ultracentrifugation. Titers ranged from 1,000 to 60,000 at the time of study. The two sera with the highest titers showed abnormal peaks at different points in the $\beta-\gamma$-globulin region upon zone electrophoresis. The component responsible for the peak in each instance was isolated; titrations in parallel disclosed that cold agglutinin activity was confined almost exclusively to this peak. Ultracentrifugation studies of the isolated components proved them to consist primarily of high molecular weight material with a sedimentation coefficient of 19S. Zone ultracentrifugation studies of the isolated components proved them to consist primarily of $19 \mathrm{~S}$ material. Zone ultracentrifugation confirmed the $19 \mathrm{~S}$ character of the cold agglutinin.

The three sera with lower titers failed to show an abnormal component by electrophoresis; however, the cold agglutinins were found to sediment as $19 \mathrm{~S}$ proteins in the density gradient system.

One case of paroxysmal cold hemoglobinuria of the
Donath-Landsteiner variety was also studied and showed "antibodies" of 19S character. This was confirmed through the use of specific antiserum to $19 \mathrm{~S} \gamma$-globulin.

In contrast, the "antibody" activity of the sera of 2 patients with acquired hemolytic anemia of the warm variety, with positive direct and indirect Coombs tests, was due to $\gamma$-globulins of $7 \mathrm{~S}$ character rather than to 19S macroglobulins. Direct Coombs reactions were obtained with the use of anti-7S human $\boldsymbol{\gamma}$-globulin rabbit serum while no visible reactions occurred with anti-19S $\boldsymbol{\gamma}$-globulin rabbit serum. Zone ultracentrifugation of the sera in these cases also showed the $7 \mathrm{~S}$ nature of the "antibody" protein.

Studies on the Ground Substance in Myxedema. II. Effect of Desiccated Thyroid, L-Triiodothyronine and Cortisone in Myxedema, Localized Myxedema, and Severe Exophthalmos. J. LESTER GABRILOVE, New York, N. Y. (Introduced by Louis J. Soffer).

Without anesthesia skin was obtained at frequent intervals from the same area by punch biopsy and studied histologically and histochemically. The metachromatic material present in the skin of myxedematous patients disappeared during the course of treatment with desiccated thyroid and reappeared when specific therapy was withdrawn. The administration of 1-triiodothyronine caused a more rapid disappearance of the metachromatic material than was noted with the use of desiccated thyroid. Similarly when 1-triiodothyronine was withdrawn, the histochemical appearance characteristic of myxedema promptly recurred. The amount of metachromasia in the myxedematous skin was also decreased by the systemic administration of cortisone alone.

The simultaneous occurrence of localized (pretibial) myxedema and malignant exophthalmos is common. It has been reported from this laboratory that in experimental exophthalmos there is an increased deposition of mucoprotein. The injection of hyaluronidase into an area of pretibial myxedema or generalized myxedema rapidly resulted in pitting of the lesion and a decrease in the amount of metachromatic material demonstrable on histologic examination. A single injection of cortisone or of 1-triiodothyronine into the region involved in pretibial myxedema effected a decrease in the quantity of the mucinous material in the lesion over a period of several days. The systemic administration of cortisone also altered the lesion in similar fashion.

In patients with malignant exophthalmos of short duration, the edema of the lids was made to pit by the injection of hyaluronidase and caused to decrease by the systemic administration of cortisone. Biopsy of these lesions was not feasible.

The significance of these observations is discussed.

Treatment of Abnormal Regulation of Respiration in Pulmonary Emphysema by Pharmacologic Means. Morton Galdston,* New York, N. Y.

Advanced emphysema patients exhibit a diminished ventilatory response to $\mathrm{CO}_{2}$ and respiratory depression 
upon sudden relief of anoxemia by inhalation of oxygen which sometimes results in $\mathrm{CO}_{2}$ narcosis. Treatment of this complication with a mechanical respirator is often unsatisfactory; therefore, other means were investigated.

Studies with either $20 \mathrm{cc}$. intravenous Aminophylline $(0.5 \mathrm{G})$ or $0.85 \mathrm{~N}$ saline and oral Diamox $(250 \mathrm{mgm}$. every 12 hours) alone and together during inhalation of mixtures of approximately 3 and 5 per cent $\mathrm{CO}_{2}$ in $\mathrm{O}_{2}$ and also 100 per cent $\mathrm{O}_{2}$ in two patients indicate that these abnormal ventilatory responses can be altered. Aminophylline administered after the metabolic effects of Diamox ${ }^{(8)}$ had stabilized nearly doubled minute ventilation as compared with saline-control, when measured at the same level of arterial $\mathrm{pCO}_{2}$ attained with the $\mathrm{CO}_{2}-\mathrm{O}_{2}$ mixtures in the different periods. Little or no ventilatory stimulation occurred with Aminophylline or Diamox ${ }^{\circledR}$ alone. However, in an individual with a normal respiratory regulatory mechanism Aminophylline, Diamox ${ }^{(8)}$ and both together respectively caused 2-, 3- and 4-fold ventilatory increases.

Aminophylline, but not saline or Diamox $\$$, promptly overcame respiratory depression during oxygen inhalation and sustained minute ventilation 15 to 25 per cent above room air levels over the next $3 / 4$ of an hour of observation. Arterial $\mathrm{pCO}$, was then 7 to $11 \mathrm{~mm}$. $\mathrm{Hg}$ below and $\mathrm{pH} 0.06$ units above oxygen-saline period levels, when arterial $\mathrm{pCO}$ rose 3 to $10 \mathrm{~mm}$. $\mathrm{Hg}$ and $\mathrm{pH}$ fell 0.03 to 0.06 unit as compared with room air. When Aminophylline was administered with Diamox ${ }^{8}$, again arterial $\mathrm{pCO}_{2}$ fell 11 to $13 \mathrm{~mm}$. $\mathrm{Hg}$ while $\mathrm{pH}$ remained unchanged or fell only 0.03 unit. Aminophylline-stimulated respiration was still present during intermittent oxygen inhalation continued for two and one-half hours.

Plasma Concentrations of Epinephrine and Norepinephrine in Normal Individuals, with Observations on the Arteriovenous Difference and In Vitro Disappearance of the Amines. Hugo Garcia and John Wallace, Durham, N. C. (Introduced by Eugene A. Stead, Jr.).

Mental and physical activities are known to affect blood levels and rate of urinary excretion of epinephrine and norepinephrine. Subjects at rest might be expected to have a different range of normal values from subjects carrying on usual activities. Two groups of normal persons were studied: 15 subjects after 45 to 60 minutes of complete bed rest and 15 subjects during regular work. Arteriovenous differences of epinephrine and norepinephrine in a group of 30 normal individuals were also examined.

Samples were assayed fluorometrically and in duplicate, using a simplified and standardized technique based on the Aronow and Howard modification of the ethylenediamine method. Final technique was standardized after a re-evaluation of all steps involved in the original procedure and re-examination of previously raised criticisms concerning the methodology. Sensitivity permits quantitative measurement of $0.25 \mu \mathrm{g}$. epinephrine per liter plasma and $0.5 \mu \mathrm{g}$. norepinephrine per liter plasma differences. Adding mixtures of both amines to plasma sam- ples, $93 \pm 13$ per cent and $84 \pm 15$ per cent, respectively, are recovered. Method was tested in detecting amines after parenteral administration to humans and to demonstrate slow disappearance of epinephrine and norepinephrine in vitro.

Epinephrine was found present in only 2 of the 15 individuals at rest, average norepinephrine levels for the group being $2.2 \pm 0.54 \mu \mathrm{g}$. per $\mathrm{L}$. The ambulatory group showed detectable amounts of epinephrine (average 0.30 $\pm 0.21 \mu \mathrm{g}$. per L.) in 14 of the 15 subjects, with average norepinephrine concentration of $3.2 \pm 0.94 \mu \mathrm{g}$. per $\mathrm{L}$. Differences are statistically significant $(P<0.01)$. The values differ from ones reported originally by WeilMalherbe and Bone.

The venous blood had a higher norepinephrine concentration. In 30 subjects, average arteriovenous difference was minus $0.36 \mu \mathrm{g}$. per L., $\mathrm{P}<0.01$. Epinephrine levels, when detectable, showed random distribution in arterial and venous samples.

Data show: no detectable epinephrine at rest; increase of both catechols on activity; negative arteriovenous norepinephrine difference.

\section{A New Concept in Prosthetic Material for Use in Plastic Cardiac Surgery. Gofrredo G. Gensini, VICENTE N. ROGER, Robert L. Hawley, and GardNER MiddLE- BROOK,* Denver, Colo.}

No ideal prosthetic substance for plastic cardiovascular surgery has yet been found. A material specially prepared from sheep caecum and used for the manufacture of condoms was selected for investigation. This paperthin laminate membrane was chosen because of its mammalian origin, pliability and strength.

This investigation was designed to determine the extent to which this substance could be functionally incorporated into vascular tissues. The experimental animals used were dogs and this material was grafted into arteries, veins and heart. Artificial semilunar valves were constructed in aortas.

At first, leukocytes and fibrin appeared in the spaces between the laminae. Then fibroblasts rapidly migrated from the host tissue through the spaces, utilizing the laminae as a framework. Within two months replacement by host tissue was complete. This prosthetic material elicited no significant foreign body reaction. The artificial semilunar valves retained their original shape with free edges, open sinuses and appeared to have increased strength. The leaflets were slightly but uniformly thickened and somewhat smaller. Thrombotic phenomena did not occur on the heart grafts or on the valves. However, the pulmonary artery grafts became thrombosed.

The rate of fibrosis of the prosthesis appears to be adequate to insure retention of the graft. This is probably due to guiding of the proliferating fibroblasts through the interstices of the laminae, resulting in a laminated structure with great strength.

The concept of a cardiovascular prosthesis being completely replaced by multi-layered fibrous connective tissue 
of the host appears to represent an advance over previously used irreplaceable, nonlaminated substances. The material described seems, in particular, to possess superior qualities for the construction of artificial cardiac valves.

The Anabolic Significance of the Fecal Dipyrrylmethene Pigments or Mesobilifuscin Group. A. SIGRID GILBERTSEN and P. T. Lowry, Minneapolis, Minn. (Introduced by C. J. Watson).

The compound which Siedel designated as mesobilifuscin (Mbf) is found in the feces partly as a dark brown pigment, partly as a colorless chromogen readily converted to the pigment. Infra-red spectroscopic study of this and of the fecal pigment earlier designated as copronigrin has revealed close, if not complete, identity. Siedel's analysis of the fecal Mbf and of that obtained by appropriate treatment of mesobilirubinogen, in vitro, indicates a dipyrrylmethene structure, in contrast to the tetrapyrrylmethene chain of the bilirubinoids. It has been generally assumed that the fecal dipyrrylmethene is derived by oxidative schism of bilirubinoid pigments. In the present study, however, the labelling of $\mathrm{N}^{15}$ mesobilifuscin following administration of $\mathrm{N}^{15}$ glycine, unlike that of stercobilin, is largely limited to the early period, being small or relatively insignificant in comparison with that of stercobilin, at the time of destruction of mature circulating erythrocytes. This indicates that the compound is mainly anabolic rather than catabolic in origin and that it may be derived from unused pigment in the sense of a pigment "complex" or pool, such as first suggested by Whipple in 1922. Further indication of anabolic character is provided in the comparison of amounts of fecal urobilinogen and $\mathrm{Mbf}$ in cases of refractory or aplastic as contrasted with hemolytic anemia. In the former the values are much below normal, even when the urobilinogen is normal; in other words, the $U / M b f$ ratio is characteristically large. In hemolytic anemia the amounts are large and the $\mathrm{U} / \mathrm{Mbf}$ ratio is normal or reduced.

In ten cases of liver disease thus far studied the fecal Mbf has been remarkably low. The possible significance of this will be discussed.

Cellular Damage Associated with the Delayed Hypersensitive State in Mumps Virus Infection. LownLL A. Glasgow and Herbert R. Morgan,* Rochester, N. Y.

A state of delayed hypersensitivity to mumps virus develops during the disease in man and a similar state has been produced in guinea pigs infected with mumps virus. In either host, this hypersensitive state is manifested in vivo by a delayed type of dermal reaction to the injection of heat-inactivated virus. In the guinea pig it can be shown that dermal sensitivity is associated with a cellular hypersensitivity by studies in vitro. Explants of splenic tissue from such animals are cultivated on a plasma coagulum in a medium consisting of guinea pig serum, ox serum ultrafiltrate, and a balanced salt solution. From such explants, macrophages migrate into the surrounding plasma coagulum. With the addition of mumps viral antigen there is a marked inhibition of the migration of macrophages from the splenic tissue, and after continuing contact with the antigen these cells round up, become vacuolated, and finally undergo lysis. Similar exposure of splenic tissue from normal guinea pigs to mumps viral antigen is without effect. This cytotoxic action of the mumps viral antigen can be abolished by addition of cortisone to the culture medium prior to the introduction of the viral antigen.

The implications of these findings in the natural disease in man, in which the hypersensitive state can be shown to be present during the development of symptoms, will be discussed from the standpoint of the possible role of this state in the symptomatic manifestations of the disease through the cellular damage produced by the interaction of viral antigen and the hypersensitive cell.

\section{A Comparison of Certain Aspects of Calcium Kinetics in} Hyperparathyroidism and Paget's Disease of Bone. RICHARD Goldsmith, Cincinnati, O. (Introduced by Morton Hamburger).

Hyperparathyroidism and Paget's disease of bone are believed to be associated with increased formation and destruction of bone. The present investigation was initiated to provide a better understanding of the abnormal physiology of these two diseases and the differences between them. Calcium studies utilizing balance and radioactive tracer techniques were conducted on 3 patients with active primary hyperparathyroidism (one had $\mathrm{X}$-ray bone disease) and on 2 patients with active, widespread Paget's disease. Each patient was in negative calcium balance when studied. The determinations of stable and radioactive calcium content were performed on serum, urine and stool. The apparent size of the available calcium pool in each patient with Paget's disease was more than ten times greater than in each subject with hyperparathyroidism. The size difference was obvious within a few hours after IV administration of radiocalcium and increased with time. The total excretion of radiocalcium by the patient with Paget's disease studied for the longest time interval was 4.3 per cent of the administered dose; the excretion in the patient with hyperparathyroidism studied for a similar interval was 20.6 per cent of the administered dose.

One patient with Paget's disease was given 500 milligrams of cortisone daily to see what effect this antianabolic agent would have on calcium metabolism. The degree of negativity of the calcium balance was considerably reduced and the excretion of labeled calcium ceased. A hyperparathyroid subject similarly studied with 300 milligrams of cortisone daily showed no change. The most likely explanation for all of these findings is that in Paget's disease there is a much slower movement of calcium between bone forming and bone resorbing surfaces than in hyperparathyroidism. This is true in the patients studied even though bone resorption in both was equally enhanced as evidenced by equivalent degrees of negative calcium balance. These data also suggest that the mechanism of the bone resorption in the two diseases 
is different. Finally, it seems most consistent with the data to assume that cortisone benefits the patient with Paget's by slowing bone breakdown.

Delayed Allergy and Agammaglobulinemia. RoBert A. Good,* Solomon J. ZaK, Donna R. Jensen, and A. M. Pappenheimer, JR., Minneapolis, Minn.

Much interest concerns the nature of delayed allergy. Particularly incisive in the study of relationships of bacterial allergy to other immunologic mechanisms are agammaglobulinemic patients. Chosen for this study were six male children with congenital and three adults with acquired agammaglobulinemia. In spite of prior observations indicating that these patients lack delayed allergy to pneumococcal and streptococcal antigens, the present studies indicate, in agreement with Porter, that agammaglobulinemic patients can develop bacterial allergy.

1. Bacterial allergy to streptococcal products and tuberculin is transferred with cells to agammaglobulinemic patients in whom it persists for two years, indicating that these patients can sustain bacterial allergy.

2. Delayed allergy to 2,4-dinitrofluorobenzene is regularly produced by its application to skin of either normal or agammaglobulinemic patients. This form of allergy is regularly transferred from sensitized normal or agammaglobulinemic patients to non-sensitized recipients with large numbers of viable circulating leukocytes.

3. Normal and agammaglobulinemic adults and children are regularly sensitized to diphtheria toxoid by the intradermal injection of specific diphtheria toxoid-horse antitoxin precipitates. No sensitivity to horse antitoxin is produced.

4. Conversely, agammaglobulinemic patients and normal persons develop dermal sensitivity to horse gamma globulin when the latter is injected as specific precipitate with anti-horse gamma globulin rabbit serum. No sensitivity to rabbit serum is produced.

The delayed allergy developed by these means may be transferred from sensitized normal or agammaglobulinemic patients to normal persons by injection of viable leukocytes from members of either group.

These observations indicate that the mechanism for development of delayed allergy is intact in agammaglobulinemic patients and tend to dissociate bacterial allergy from classical antibody production. Their implication to immunological theory and their relationship to the occurrence of fibrinoid disease and arthritis in agammaglobulinemic patients will be considered.

Further Studies of the Use of the Ear Oximeter for Repeated Measurement of Cardiac Output at Rest and During Exercise. Allan V. N. Goodyer * and AlLEN Chetrick, New Haven, Conn.

The determination of cardiac output by means of dye dilution curves recorded by ear-densitometry has required calibration of each curve either by one or more arterial samples, or by a 5-minute venous sample. The arterial cannulation adds difficulty and discomfort to the method, and the use of the 5-minute sample involves not only a large extrapolation from a non-linear region of the cuvette response curve, but also a degree of stability of the oximeter baseline which has, in our experience, proven very difficult to obtain, particularly during exercise.

The present study explored the use of a standard calibration factor for one earpiece, based on the means of the peak values of 22 simultaneous oximetric and arterial dye curves in 12 subjects. The correlation between paired peak values was found to be quite good $(r=+0.81$, $\mathrm{S} . \mathrm{E} . \mathbf{= 0 . 2 1}$ ). If only repeated determinations in the same subject are considered, the correlation of simultaneous peak values was even better $(r=+0.92$, S.E. $=0.33)$.

Simultaneous determinations of cardiac output with the above method and by the Fick procedure, both at rest and during exercise, were closely comparable $(r=+.91$, $\mathrm{S.E} .=0.28$ ). Indigo Carmine (as reported by Newman), but not Methylene Blue, was found to be a good substitute for T1824. The mean error of the dye method was -4 per cent \pm 16 per cent, as compared to the Fick method.

The ear oximeter, therefore, used with a standard calibration factor, and with certain other precautions, would appear to be applicable to the repeated measurement of large changes of cardiac output during exercise in human subjects, particularly where relative values in the same subject are concerned.

The Effect of Acetazolamide (Diamox) on Calcium and Citrate Metabolism in Humans. EDwin E. GoRdon and Sheldon G. Sheps, Boston, Mass. (Introduced by A. S. Freedberg).

The physico-chemical properties of calcium and citrate in aqueous solution, and in blood, have been investigated intensively. It has been postulated that the citrate present in urine binds calcium with the formation of a soluble and poorly dissociable complex, thus preventing the precipitation of insoluble calcium salts. Harrison and Harrison have recently provided experimental evidence to support this view, demonstrating a marked reduction in urinary citrate excretion with precipitation of calcium salts in the urinary tract of rats which were fed acetazolamide (Diamoxi).

We have studied the citrate and calcium concentrations in the blood and urine of a patient who developed urolithiasis while receiving Diamoxi. In addition, citrate and calcium metabolism was studied in five normal subjects before, during and after Diamox administration.

Diamox' (250 mgm. t.i.d. for three days) reduced the urinary citrate excretion in all the subjects. In two subjects, citrate excretion was reduced to 10 per cent of control levels; in the remaining four subjects a fall to 25 to 33 per cent of control values was observed. Statistical evaluation of these data showed a $z$ value which falls beyond the 0.001 level of significance. Serum citrate and calcium concentrations and urinary calcium content were not altered. Diamox administration thus increased the calculated ionized calcium in the urine (potentially precipitable calcium) from an average 
value of 51 per cent to an average value of 93 per cent of total urinary calcium. This effect of Diamox. was apparent six hours after the administration of a single dose of the drug.

Further studies to clarify the mode and site of action, the dose relationships and the duration of effect of Diamox ${ }^{(B)}$ on citrate and calcium metabolism are currently in progress.

The Effect of Nitroglycerin on the Coronary Circulation and Myocardial Metabolism in Man. RICHARD GoRLIN,* JoHn M. Bozer, and John W. Vester, Boston, Mass., Portsmouth, Va., and Pittsburgh, $\mathrm{Pa}$.

The capacity of the coronary vascular bed to dilate following administration of nitroglycerin has been studied in patients with mild and severe heart disease of varied etiology. Following coronary sinus catheterization the nitrous oxide technique was utilized to measure coronary blood flow in 12 patients with normal left ventricular (LV) work and minimal symptoms, and in 3 patients with greatly increased LV work. Nearly simultaneous estimations of coronary flow, myocardial oxygen consumption, cardiac output (Fick or indicator dilution method), systemic arterial pressure, and heart rate were made before and 10 minutes after $0.6 \mathrm{mg}$. nitroglycerin sublingually.

A linear correlation $(R=0.81, p=.01)$ has been found between myocardial oxygen consumption per second of systole and resting $\mathrm{LV}$ work in $\mathrm{Kg}$. $\mathrm{M}$ per min. per $\mathrm{M}^{\mathbf{2}}$. Oxygen consumption increased not only with increased work but also (for the same work) directly with heart rate and the number of seconds spent in contraction per minute. In the nearly normal patients, following nitroglycerin, both coronary flow and myocardial oxygen consumption increased ( 57 per cent) while coronary vascular resistance (CVR) decreased 50 per cent. Increase in oxygen consumption was out of proportion to minor changes in work or heart rate. Calculated cardiac efficiency decreased. In the patients with increased LV work, CVR and therefore coronary flow and myocardial oxygen consumption did not change significantly; cardiac work decreased disproportionately and efficiency declined. The fixed coronary resistance and flow in the severe cardiacs may indicate that maximal coronary vasodilatation had already been reached in response to the greatly increased work loads and oxidative energy demands.

These studies would suggest that nitroglycerin altered myocardial energy metabolism and that either coronary flow increased secondarily or that cardiac work decreased when coronary flow could not be increased.

Psychological Factors and the Manifest Development of Leukemia and Lymphoma in Humans. WIILIAM A. Greene, JR., Rochester, N. Y. (Introduced by George L. Engel).

Proponents of any one factor, virus, chemical, radiation, or hormone as an initiating etiological agent in leukemia or lymphoma consider the probability that other factors may play a precipitating role, perhaps through modification of host resistance.

Study of 100 patients has shown that the disease developed in a setting of separation with depression in 93 of 100 patients. Separation is considered to be a psychophysiologic state occurring in situations of loss of a significant person or goal with an accompanying affect of conscious or unconscious sadness or hopelessness and usually associated change in motor, gastrointestinal, and vascular activity.

Unselected patients showed the following kinds and frequency of unresolved separation occurring at a median point of 12 months before apparent onset in the adults and 6 months before apparent onset in the children.

(1) Men: 22 patients; leukemia 12, lymphoma 10. Separations for the men included loss of a significant person, mother, wife, father, advent of an offspring, a daughter leaving home, change of job, or retirement. Such separations had occurred in 20 of the 22 men.

(2) Women: 40 patients; leukemia 22, lymphoma 18. Separations for the women included loss of a significant person, mother, husband, father, child, the menopause and change of home. Such separations had occurred in 38 of the $\mathbf{4 0}$ women.

(3) Children : 38 patients; leukemia 33, lymphoma 5. Separations for the children included birth of a sibling rival, change of home, change of school, loss of father, loss of a substitute mother. Such separations had occurred in 35 of the 38 children.

These data indicate that many of the prodromal symptoms are related to psychological processes as well as to the developing lymphoma or leukemia. Further, it is considered that significant separation may be a frequent condition precipitating the manifest development of leukemia and lymphoma.

In Vivo Capillary Alterations in Man During Hemorrhagic Fever and Other Infectious Illnesses. SHEcDoN E. Greisman, Baltimore, Md. (Introduced by Charles L. Wisseman, Jr.).

Clinical and pathologic evidence points to a significant degree of small vessel dysfunction in certain infectious diseases such as epidemic hemorrhagic fever and some rickettsial infections which per se might explain important features of these diseases. In order to study the dynamic state of the small vessels and to correlate this with the clinical and pathologic picture, serial direct microscopic observations of the nailfold capillaries during the course of illness were performed in 71 patients with hemorrhagic fever and 51 patients with various other infectious illnesses. The nailfold capillary bed, studied under standardized conditions, provided a segment of the minute vascular system anatomically and functionally comparable in all patients. Although individual variations were marked, certain patterns emerged:

1) Hemorrhagic fever: Decreased vasomotor activity, refractoriness to 1-nor-epinephrine, increased mean capillary diameter, and hemorrhagic diathesis characterized the febrile and hypotensive phases. Slowing of blood 
flow and sludging were less conspicuous. During the hypertensive and diuretic phases, capillary constriction and heightened vasomotor activity developed, gradually reverting to normal during convalescence.

2) Other infectious illnesses: Most other patients, despite pyrexia to $105^{\circ} \mathrm{F}$, exhibited no significant nailfold capillary alterations. However, two subjects with clinical evidence of generalized and severe capillary injury during scrub typhus and Rocky Mountain spotted fever, and one patient with infectious mononucleosis, manifested serial capillary changes indistinguishable from those noted during the hypotensive phase of hemorrhagic fever. Nine patients with early $Q$ fever failed to exhibit any significant nailfold capillary changes, conforming with the concept that $R$. burneti lesions are limited to the pulmonary area in man.

The findings indicate that the nailfold capillary reaction to a variety of infectious agents follows a definite pattern which can be correlated with the clinical and pathologic features of the illness.

Effects of Corticotropin, Hydrocortisone, and Stress on Albumin Synthesis and Catabolism in Man. JАС Grossman, A. Aaron Yalow, and Raymond E. Weston,* New York, N. Y.

The effects of stress and/or adrenal corticoids on albumin, in contrast to general protein, metabolism have received little attention. Because the use of $\mathrm{I}^{100}$-labelled human serum albumin (RISA) permits, in the absence of severe disequilibration, independent estimation of albumin breakdown and synthesis, the presumed anti-anabolic, as well as catabolic, effects of adrenal cortical hormones on albumin may be studied. The catabolic rate is the daily percentage decrease of retained radioactivity, measured from urinary $\mathrm{I}^{181}$ excretion, while anabolism is reflected by the percentage rate of decrease in specific activity of plasma albumin.

Sixteen to eighteen days following intravenous injection of RISA, to permit equilibration plus adequate control periods, corticotropin ( 80 to $120 \mathrm{U}$ daily) or hydrocortisone (200 to $300 \mathrm{mg}$. daily) was administered for 6 to 9 days to nine subjects (normal or cardiac) under metabolic ward control, including fixed dietary and fluid intake and moderate activity. Balances of electrolytes and nitrogen and daily urinary excretions of creatinine and 17-hydroxycorticoids were measured.

Control albumin turnover varied from 0.023 to 0.057 per day; synthesis approximated breakdown. Hormone administration was associated with prompt, sustained increase in urinary $I^{181}$ excretion to almost twice the control rate, reflecting increased albumin breakdown, followed by gradual subsidence despite continued therapy. Anabolic effects were delayed several days. Albumin synthesis increased on corticotropin, except in one subject, but was unchanged or slightly decreased on hydrocortisone. In two patients who developed spontaneous infections, markedly increased breakdown and severely diminished synthesis of albumin occurred.

The apparent contribution of the increased albumin catabolism to the negative nitrogen balance was unusually large relative to the ratio of exchangeable albumin to total body protein. The implications of this observation will be discussed.

Demonstration of Erythropoietin in Human Plasma. Clifford W. Gurney, Eugene Goldwasser, Leon O. Jacobson,* and Chao Pan, Chicago, Ill.

Demonstration of plasma erythropoietin activity has been facilitated by the finding that hypophysectomized or acutely starved rats give an exaggerated response to fractionated animal or human anemic plasma. It has been demonstrated repeatedly that the increased incorporation of $\mathrm{Fe}^{50}$ in rat erythrocytes is a reliable index of erythropoietic activity induced by injections of such fractionated plasma. Two 2-ml. injections of heat-denatured or ethanol-extracted normal human plasma failed to increase the per cent of $\mathrm{Fe}^{\mathrm{50}}$ incorporated into the erythrocytes $16 \mathrm{hrs}$. after injection of a tracer dose of $\mathrm{Fe}^{80} \mathrm{Cl}_{8}$. However, ethanol-extracted normal human plasma, concentrated 30 times, enhanced erythropoietic activity, increasing $\mathrm{Fe}^{50}$ incorporation two fold. Unconcentrated extracts of heat-denatured plasma from a patient with hypoplastic anemia consistently produced a three- to five-fold increase in $\mathrm{Fe}^{\mathrm{50}}$ uptake in hypophysectomized or starved rats. After transfusion raised the hemoglobin level, plasma activity could no longer be demonstrated. Activity was demonstrated in plasma from patients with pernicious anemia, blood loss anemia, aleukemic leukemia, Hodgkin's disease, acute hemolytic anemia, and hypoplastic anemia. It was not found in plasma from anemic patients with uremia, advanced neoplasms, or malnutrition. If the anemia is mild, concentration of the extract often permits demonstration of activity. Increased erythropoietin concentration has also been demonstrated in the non-dialyzable fraction of urine from some anemic patients. We conclude that erythropoietin is present in normal plasma and that its concentration is increased in plasma from patients with a variety of anemias. Our data are consistent with the concept that erythropoietin is a hormone that regulates the rate of erythropoiesis. The anemic state usually induces a compensatory increase in this factor. Suboptimal levels or failure to respond to anemia by increasing the plasma erythropoietin concentration is postulated to be of importance in the pathogenesis of some anemias.

Peripheral Arteriovenous Shunting Mechanisms in $\mathrm{Pa}$ tients with "S" Type Hemoglobin. E. RICHARD HaLden, Brian J. Sproule, and E. E. Mutrhead,* Dallas, Tex.

During investigation of the in vivo $\mathrm{pO}_{2}$-sickling relationship, marked variation in per cent sickling occurred in different venous blood samples from the same subject. Possible causes of the phenomenon were studied in 18 subjects possessing S-type hemoglobin.

Arterialization of venous blood was demonstrated in 15 subjects by analyzing simultaneously drawn peripheral venous, arterial and central venous ( 7 subjects) blood for 
$\mathrm{pO}_{2}$ (polarograph), oxygen saturation and degree of sickling. Pure oxygen breathing was used to render peripheral arteriovenous admixture more apparent.

Nine individuals showed increased oxygenation of venous blood while breathing room air $\left(\overline{\mathrm{SvO}_{2} 84}, \overline{\mathrm{PvO}_{2}} 55\right.$, $\left.\overline{\mathrm{SaO}}_{2} 91, \overline{\mathrm{PaO}}_{2} 68\right)$. Oxygen breathing caused marked increase in saturation of venous blood and in $\mathrm{PvO}_{2}$ (175 $\mathrm{mm} . \mathrm{Hg}$ ) in five. In the remaining four no increase occurred.

Six other subjects showed no evidence of peripheral arteriovenous admixture during ambient air breathing $\left(\overline{\mathrm{SvO}_{2}} 60, \overline{\mathrm{PvO}_{2}} 32, \overline{\mathrm{SaO}_{2}} 93, \overline{\mathrm{PaO}_{2}} 74\right)$ but did show such admixture during pure oxygen breathing $\left(\overline{\mathrm{SvO}_{2}} 89\right.$, $\left.\overline{\mathrm{PvO}}_{2} 69\right)$.

Arterialization was most often demonstrated in veins of the forearm and was influenced by the concentration of inspired oxygen. Inhalation of pure oxygen by 5 subjects resulted in a fall in peripheral $\mathrm{SvO}_{2}$ (mean decrease 16 per cent) but caused an increased saturation of simultaneously drawn pulmonary artery blood.

Twice during low oxygen and once during room air breathing $\mathrm{SvO}_{2}$ was higher than $\mathrm{SaO}_{2}$. The degree of sickling was correspondingly less in the venous than in arterial blood, suggesting that segregation of desaturated sickled cells from more oxygenated discoid cells occurred in $\mathrm{A}-\mathrm{V}$ communications.

Individuals possessing S-type hemoglobin appear to have functional peripheral $\mathrm{A}-\mathrm{V}$ communications which, along with the established relationship of sickling to $\mathrm{pO}_{2}$, offer a possible explanation of discrepancies in peripheral sickling in such patients.

Studies on the Spinal Fluid and Serum Protein Electrophoretic Pattern in Patients with Diabetes Mellitus. Wirliam J. Hammack, Willard R. Starnes, and S. Richardson HiLl, JR., Birmingham, Ala. (Introduced by Ben Friedman).

Because of the elevated spinal fluid protein levels and abnormal serum protein patterns present in some patients with degenerative complications of diabetes mellitus, studies have been undertaken to characterize further the serum and spinal fluid protein patterns in forty such patients. Electrophoretic studies were simultaneously performed on serum and concentrated spinal fluid. The results have been expressed in per cent and milligrams. In diabetic patients without degenerative complications, the protein patterns of the serum and spinal fluid were normal. Patients with diabetic neuropathy had a normal serum protein pattern, but an elevated spinal fluid protein, consisting of increases in each fraction, especially in gamma globulin. This may indicate the presence of other mechanisms, in addition to a generalized increase in vascular permeability. Patients with diabetes mellitus and persistent proteinuria had a lowered spinal fluid total protein and albumin fraction, but an elevated alpha and beta globulin fraction. The serum was characterized by a decreased albumin and an increased alpha and gamma globulin. The decrease in the spinal fluid total protein and albumin fractions possibly reflects the de- creased serum albumin level resulting from persistent proteinuria. The slight increase in the alpha fractions may result from increased glycoprotein associated with this component.

Serum Cholesterol, Diet and Stress in Patients with Coronary Artery Disease. James F. Hammarsten, Charles W. Cathey, Robert F. Redmond, and StewART Wolf,* Oklahoma City, Okla.

Careful measurements of serum cholesterol were made each week for an average of 9 and up to 14 months in a group of 12 male subjects (ages 30 to 70 ) who had survived a well documented myocardial infarction. The method gave reproducibility in duplicate samples within 2 per cent and recoveries better than 98 per cent. Each subject kept a daily record of what he ate. The diets were relatively high in cholesterol and fat content and were maintained at the same level throughout the period of study. There were no significant changes in body weight and no gross changes in the amount of exercise performed. A strong and positive patient-physician relationship developed but no therapeutic agents were administered. Nevertheless in every patient a decrease in serum cholesterol was observed over the 9 months of the study. The mean decrease for the group was 13 per cent $(p=0.001)$.

Independently of the chemical determinations, each patient was studied carefully from the standpoint of his life adjustment and his reaction to people and events in his day-by-day experiences, and judgements covering each week were recorded. In addition to the over-all downward trend of serum cholesterol, there were significant variations from week to week. The average intra-individual range was $103 \mathrm{mg}$. per cent. On 20 occasions serum cholesterol rose higher than the mean value for that individual by more than 15 per cent. It was striking that 19 of the 20 occasions of high cholesterol corresponded with periods that had been separately judged as particularly stressful for the individuals concerned.

The Cellular Basis for the Electrocardiographic Changes
Associated with Alterations in Serum Potassium. Associated with Alterations in Serum Potassium.
Hans H. Hecht,* Joseph M. Heath, Eugene S. Mater, Ramon L. Lange, and Clifford V. Nelson, Salt Lake City, Utah.

The characteristic changes in the electrocardiogram of patients with altered extracellular potassium concentration provided a means by which the role of $\mathrm{K}^{+}$in the genesis of cardiac action potentials and its role in cardiac automaticity may be assessed. This can be approached in two ways: 1) Assuming the surface electrocardiogram to represent membrane current, a monophasic curve may be constructed by double integration, which, though nonquantitative, should resemble the average time course of transmembrane potential under circumstances of abnormal $\mathrm{K}^{+}$concentrations. 2) Transmembrane potentials may be recorded directly by microelectrodes from isolated spontaneously beating hearts (bull frog) perfused 
with Tyrode solution of variable potassium content but unchanged in osmolarity.

The two approaches were carried out and yielded complementary information. Low potassium perfusion (increase in intracellular: extracellular gradient) resulted in prolongation of action potential duration and a more gradual than normal restoration of resting potential in diastole. This agreed with the double integration analysis and coincided with flattening of $\mathrm{T}$-waves, and large $U$-waves in surface potentials in man, but without demonstrable $U$-waves in frogs. The time relationships suggest, however, that under these circumstances the appearance of $U$-waves is causally related to the last phase of ventricular recovery. Increasing extracellular $\mathrm{K}^{+}$ concentration, and thereby decreasing the $\mathrm{K}^{+}$gradient, resulted in decrease in resting membrane-and actionpotential, shortening of its duration, and steepening of the final phase of recovery in the experiments with microelectrodes. The geometric analysis suggested that the conduction disturbances and QRS changes of the surface record in hyperpotassemia are the result of slowed cellular depolarization caused by a decrease in resting membrane potentials. The large hyperpotassemic $T$-waves constitute a summated effect of an increased rate of recovery. Both the geometric and the experimental analyses are consistent with an excessive cellular outflow of $\mathrm{K}^{+}$in hypokaliemic states and a smaller than normal flux in hyperpotassemia. By inference it may be stated that, as in nerve, intracellular accumulation of $\mathrm{K}^{+}$ is linked to the magnitude of resting membrane potentials of the ventricular myocardium and that this ion is concerned with cardiac recovery from the excited state.

\section{Intestinal Excretion of Cholesterol: A Mechanism for} Regulation of Plasma Levels. Leon Hellman,* RoBert S. Rosenfeld, William Insull, JR., and Edward H. Ahrens, JR., New York, N. Y.

A physiologic mechanism by which the plasma cholesterol level can be elevated or reduced has been elucidated by means of a "labelled sterol balance study." This was performed following intravenous administration of cholesterol-4- $\mathrm{C}^{16}$ to a patient with hyperlipemia and xanthoma tuberosum. Since in vivo this radiocarbon atom remains permanently fixed in the sterol molecule, measurements of excreted radioactivity accurately reflect net changes in the excretion of cholesterol and its transformation products. The following observations were made:

1. Plasma and fecal cholesterol specific activities approximate each other, thus indicating that fecal cholesterol was largely derived from a pool in isotopic equilibrium with plasma sterol.

2. Four weeks after administration of the labelled cholesterol, the patient's diet was changed from ad libitum to a 40 per cent butter oil formula. Plasma cholesterol rose from 478 to $720 \mathrm{mg}$. (net gain $7.2 \mathrm{gm}$.) and fecal excretion of cholesterol and its end-products decreased by a minimum of $5.4 \mathrm{gm}$. during this period.

3. When the butter diet was replaced five weeks later by an isocaloric $\mathbf{4 0}$ per cent corn oil formula, cholesterol fell from 720 to $340 \mathrm{mg}$. (net loss $11.4 \mathrm{gm}$.) and fecal excretion of cholesterol and its end-products increased by a minimum of $9.6 \mathrm{gm}$.

4. The plasma cholesterol specific activity curve was similar to that observed in normocholesteremic subjects. No changes in slope were seen in the successive dietary periods.

These novel findings were made possible by purposeful study of unsteady states when plasma lipide concentrations were altered by simple dietary manipulations. The data indicate that the character of the dietary lipides affects the quantity of plasma cholesterol by diminishing or increasing the fecal excretion of cholesterol and its transformation products. Basis is provided for an interpretation of hypercholesteremic states in man in which attention will be focussed primarily on intestinal excretory mechanisms.

\section{Alterations in Phosphorus Metabolism During General Anesthesia in Man. Dorothy H. Henneman and LEROY D. VANDAM, Boston, Mass. (Introduced by Francis D. Moore).}

Previously observed changes in peripheral blood concentrations of carbohydrate metabolites during ether and thiopental anesthesia in normal man have been investigated further by the administration of epinephrine $(0.01$ $\mathrm{mgm}$. per $\mathrm{Kg}$. subcutaneous) and insulin ( 0.1 unit per $\mathrm{Kg}$. intravenous).

Epinephrine with either agent increased blood glucose more than in unanesthetized subjects and to levels 150 mgm. per $100 \mathrm{ml}$. above those expected with ether alone. Elevations in organic acid concentrations (pyruvic, lactic, citric) were no more than those after epinephrine without anesthesia, or during ether anesthesia alone. Inorganic phosphorus concentrations decreased 0.1 to 0.4 mgm. per $100 \mathrm{ml}$. thirty minutes after epinephrine, but thereafter, unlike the expected unanesthetized response, steadily increased 1 to $2 \mathrm{mgm}$. per $100 \mathrm{ml}$.

Insulin during ether initially lowered blood glucose an average of $25 \mathrm{mgm}$. per $100 \mathrm{ml}$., but concentrations remained at hyperglycemic levels throughout the three-hour test period. Inorganic phosphorus levels rose after insulin rather than decreasing sharply as in the unanesthetized subject. Organic acid changes induced by insulin were no more than those expected with ether alone and after insulin in the unanesthetized patient.

The elevations in serum inorganic phosphorus are consistently associated with abnormal hyperglycemic responses to administered glucose, epinephrine, and insulin using either anesthetic agent. These abnormal responses are not associated with abnormally increased elevations in lactate; total lactate changes under these experimental conditions are never more than those expected with ether anesthesia alone. The lactate and pyruvate changes with ether might be related to an increased glycogenolysis peculiar to ether, but unrelated to the observed phosphorus changes. We suggest that ether and thiopental directly alter phosphorylating mechanisms associated with 
glucose utilization, rather than directly inhibiting glucose oxidation.

Two Mechanisms of Sustained Hypercalcemia Following Hypervitaminosis $D$ and the Milk-Alkali Syndrome. Philip H. Henneman and William H. Baker, Boston, Mass. (Introduced by Paul C. Zamecnik).

It is well known that hypercalcemia, with its attendant depression of renal function, may persist for three to twelve months following withdrawal of vitamin $D$ in hypervitaminosis $\mathrm{D}$ and following the discontinuation of milk in the hypercalcemia syndrome resulting from excessive milk and alkali ingestion. A complete balance study is presented which illustrates increased absorption of calcium continuing three months after cessation of excessive vitamin $D$ therapy. Storage of vitamin D, probably in the liver, as is known to occur in other species, presumably accounts for the persistent hypercalcemia of such patients.

A complete balance study in a second patient, performed five months after discontinuation of excessive vitamin $\mathrm{D}$ intake, illustrates persistent hypercalcemia not due to increased calcium absorption. Calcium ${ }^{45}$ has been administered intravenously to this patient and to two patients with visible metastatic calcification due to excessive milk and alkali intake. The rate of calcium ${ }^{45}$ disappearance from the blood was greatly accelerated in all three patients. This rapid decline of blood specific activity was not due to increased excretion. The calcium "pool" (per cent of calcium retained in the body/specific activity of serum) at nine days was increased from normal values of 10 to $20 \mathrm{gms}$. to $42 \mathrm{gms}$. in the patient with hypervitaminosis $\mathrm{D}$ and to $112 \mathrm{gms}$. in one of the patients with metastatic calcification. Soft tissue calcification, which may or may not be visible by X-ray during life, presumably accounts for this increase in exchangeable calcium. It is suggested that such soft tissue calcification is produced at high serum calcium levels. Following withdrawal of milk or vitamin D, resorption of these soft tissue deposits of calcium may be sufficiently rapid to maintain hypercalcemia for prolonged periods.

\section{The Suppression of Hyperadrenocorticism by Amphenone} in Man. R. Hertz, D. M. Bergenstal,* H. A. Lubs, and S. J. JACKson, Bethesda, Md.

Amphenone (1,2-bis ( $\mathrm{p}$-aminophenyl) 2-methyl-1 dihydrochloride) produces an abrupt and reversible suppression of corticoid output in eucorticoid patients as well as in cases of Cushing's syndrome attributable either to adrenal cancer ( 7 cases) or adrenal hyperplasia ( 2 cases). This suppression is manifested by: (a) decrease in both plasma and urinary corticoids, (b) reduction in blood pressure, (c) reduction in steroid diabetes, (d) control of mental aberrations attributable to excessive endogenous corticoids, (e) a variable decrease in urinary 17-ketosteroids. This suppression of corticoid output is promptly reversed upon cessation of amphenone administration.
In two cases an excessive rebound effect was manifested by extreme exacerbation of hyperadrenocorticism.

In these brief courses of amphenone treatment ranging from one to 32 days, no significant effect upon the clinical progression of adrenal carcinoma was noted.

The toxic effects of amphenone, notably somnolence, drug rash, and methemoglobinemia, limit its clinical application to short-term use either for diagnostic purposes or for the acute control of the clinical and metabolic features of hyperadrenocorticism.

The Pentose Phosphate Pathway in Human Metabolism. Howard H. Hiatt, Boston, Mass. (Introduced by H. L. Blumgart).

Although there is now much evidence demonstrating the importance of the pentose phosphate pathway (PPP; hexose monophosphate shunt) in many biological processes, no information exists concerning its function in man. The role of the PPP in human metabolism was explored in two ways: 1) a study of the mechanism of ribose synthesis in a human carcinoma cell (strain $\mathrm{HeLa}$ ) grown in tissue culture; and, 2) a study of the effects and fate of $\mathrm{D}$-ribose given human subjects.

Degradation of nucleic acid ribose isolated from $\mathrm{HeLa}$ cell grown in the presence of glucose-1-C ${ }^{14}$ revealed radioactivity mainly in the first carbon atom of the ribose molecule; ribose from cells exposed to glucose-2-C $C^{14}$ was predominantly labeled in carbons 1 and 2 . Such isotope distribution is consistent with ribose synthesis via the PPP.

Twenty-four patients without liver disease infused intravenously with D-ribose, 200 or $400 \mathrm{mg}$. per $\mathrm{Kg}$. body weight, had half-times of ribose disappearance of $18 \pm 2$, or $26 \pm 3$ minutes, respectively. Half-times of $31,33,35$, and 47 minutes were observed in four subjects with hepatic insufficiency. Urinary excretion averaged 8 per cent of the infused sugar, and an average volume of distribution of 28 per cent of body weight was found. Ribose disappearance was accompanied by an average decrease in blood glucose of 33 per cent, and in six of ten subjects by a fall in plasma inorganic phosphate.

The mechanism of D-ribose assimilation was studied by isolating and degrading liver glycogen glucose from mice given ribose-1-C $\mathrm{C}^{\mathrm{d}}$ intraperitoneally. Radioactivity was found primarily in glucose carbons 1 and 3. Of the known reactions in the intermediary metabolism of carbohydrate only those of the PPP can explain this isotope distribution.

The reactions of the pentose phosphate pathway responsible for ribose biosynthesis in man and for ribose metabolism in mammals will be discussed.

Estimation of the Retinal Venous Blood Oxygen in Man by Photographic Means. J. B. HICKAM * and R. FrAYSER, Durham, N. C.

Many pathologic changes in the optic fundus, including some which occur in systemic disorders, are ascribed to circulatory insufficiency, but sometimes with 
tenuous evidence. For evaluating the role of retinal vascular insufficiency in various retinopathies, it would be highly desirable to have a means of measuring the per cent oxygen saturation in the retinal venous blood of man. The present communication describes the development of a method for accomplishing this by means of fundus photography.

The method employs the principle that per cent oxygen saturation of blood can be related to the relative intensities of reflected red and infra-red light. Relative light intensities can be measured from the density of the images produced on infra-red sensitive film. With blood in a cuvette, the per cent oxygen saturation of thirtynine samples from nine subjects, ranging from 100 to 50 per cent saturation, was photographically measured, using the fundus camera. Oxygen saturation bore a linear relation to the difference between film densities produced by reflected red and infra-red light, with a standard deviation from regression of 4.5 per cent saturation for the original negatives and 6.4 per cent for 8-diameter enlargements. In the optic fundus itself, measurements have been made on enlarged images of the retinal vessels as they cross the disc. With such thin vessels on a near-white reflecting background, calculations appropriate to transmitted light can be applied. The method is being calibrated on retinal arteries, the blood oxygen saturation of which can be varied within wide limits by having the subject breathe different gas mixtures. On four normal subjects, the per cent oxygen saturation of retinal venous blood has averaged 69 per cent (range 60 to 75 per cent). Rapidly reducing arterial oxygen saturation by about 25 per cent causes almost as great a reduction in venous blood oxygen concentration. The method appears useful, and it is being applied to the study of appropriate problems.

The Effect of an Antibody to Properdin on Hemolysis of Erythrocytes from Patients with Paroxysmal Nocturnal Hemoglobinuria (PNH). CARL F. HINz, JR., Cleveland, O. (Introduced by Austin S. Weisberger).

Previous studies demonstrated that properdin, a normal serum protein, is required for the in vitro hemolysis by normal serum of erythrocytes from patients with $\mathrm{PNH}$, but not for immune hemolytic reactions. Recently an antibody to human properdin (Anti-HP) has been produced in rabbits by Pillemer, Hinz, and Wurz. Anti-HP inactivates properdin isolated from serum or properdin in serum but does not inactivate complement. It is stable at $56^{\circ}$ and inactivates properdin even at $1^{\circ} \mathrm{C}$ in the absence of complement. Anti-HP capable of inactivating 300 units of properdin per $\mathrm{ml}$. of antiserum has been produced.

Addition of Anti-HP to normal human serum markedly decreases the ability of that human serum to hemolyze $\mathrm{PNH}$ cells in vitro, but has little effect on immune isohemolysis (Anti-A), which does not require properdin. Further addition of properdin to the human serum restores its hemolytic activity which can again be abolished by further addition of Anti-HP, thus demonstrating the specific effect both of properdin and Anti-HP. The effect of Anti-HP on PNH hemolysis is quantitatively similar to the effect of Anti-HP on properdin when measured in the zymosan assay. The inhibitory property of anti-HP against $\mathrm{PNH}$ hemolysis is removed by adsorption with properdin or the insoluble properdinzymosan complex, but Anti-HP is not affected by adsorption with reagents which do not contain properdin. These observations confirm the specificity of the antibody.

This effect of an antibody specific for properdin confirms the requirement for properdin in $\mathrm{PNH}$ hemolysis, and presents a new means of studying the in vitro interactions of properdin with $\mathrm{PNH}$ erythrocytes and other substrates. It may eventually prove useful for studying in vivo the role of properdin in $\mathrm{PNH}$ hemolysis and in other reactions in which properdin participates.

\section{A Plasma Factor That Protects Against the Lethal Ac- tion of Endotoxin. Monto Ho and Edward H. Kass,*} Boston, Mass.

Because of indications that endotoxins play a role in the pathophysiology of many disease states, exploration of some mechanisms of resistance to these bacterial substances was undertaken. One such mechanism, hitherto undescribed, is presented.

1. The lethality of crude endotoxins from Gram negative bacteria ( $S$. typhi Ty 2, S. typhi 0901 and E. coli $0127: B 8)$, prepared by killing bacterial suspensions with chloroform, was attenuated when these endotoxins were incubated with normal human plasma fractions and injected intracardially into rats. The protective effect was still demonstrable when the endotoxin of chloroformkilled $S$. typhi Ty 2 was partially purified by trypsin digestion, and alcohol and ether precipitations. However, there was no protection against highly purified endotoxins prepared by the Boivin trichloracetic acid extraction method from the same organisms.

2. Protection against intracardiac injection of the partially purified Ty 2 endotoxin was also obtained with rabbit and rat plasmas. In human plasma, this protective effect was most marked in Fractions III and IV (Cohn), and minimal or absent in Fractions I, II and V. Significant protection was evident with active fractions equivalent to as little as $0.025 \mathrm{ml}$. of plasma.

3. The plasma protective factor was non-dialyzable, stable at $60^{\circ} \mathrm{C}$ for 30 minutes at $\mathrm{pH} 7$, and resistant to repeated freezing and thawing, but was destroyed by boiling. Solutions of pure properdin, in amounts comparable to that found in the plasma fractions used, gave little or no protection. The protective factor was also independent of antibody.

4. Preliminary observations suggest that the inactivation of endotoxin by plasma fractions may be enzymic. The data indicate a mechanism of resistance against a crude bacterial product such as may be found in natural infections. When this product is "purified" by certain methods, the protective mechanism herein described is no longer active. 
The Effect of Induced Hypocalcemia on Urinary Phosphorus Excretion in Dogs. JaMES F. HoLLAND, ROBERT Brenner, Elton Rock, and Ethel Danielson, Buffalo, N. Y. (Introduced by David K. Miller).

Suppression of parathyroid secretion was postulated by Howard $e t$ al. when they demonstrated decrease in urinary phosphorus excretion after induced hypercalcemia in man. We had undertaken administration of ethylene diamine tetra acetate (EDTA) infusions ( $\mathrm{pH} \mathrm{7.4)}$ to dogs to demonstrate a possible hypocalcemic stimulus to parathyroid secretion.

Decrements in serum calcium concentration of 1.2 to $6.0 \mathrm{mg}$. per cent (median 3.6) were produced in 15 experiments in 12 normal dogs immediately after EDTA infusion of 65 to $125 \mathrm{mg}$. per $\mathrm{Kg}$. (median 100) lasting 5 to 17 minutes (median 10). Glomerular filtration rate (GFR) measured after initial load and sustaining infusion of creatinine was essentially constant throughout each experiment.

The per cent of filtered phosphorus reabsorbed in 9 experiments on 8 dogs was decreased 6.5 to 32.2 per cent below baseline (median 20.0 per cent). Inhibition of phosphorus reabsorption began in 20 to 40 minutes and was maximal in 40 to 140 minutes. Maximal phosphate excretion was 0.2 to $0.55 \mathrm{mg}$. per min. (median 0.35) above baseline. In 6 experiments on 5 dogs (one dog from above group) phosphorus reabsorption increased 3.8 to 17.8 per cent (median 9.8) above baseline and excretion decreased. Amount of fall in calcium and variations in GFR were apparently unrelated to amount or direction of change in phosphorus reabsorption. Six experiments on 4 thyroparathyroidectomized animals gave inconclusive results.

The data demonstrate that increased phosphorus excretion occurs in a majority of instances after acute hypocalcemia induced by EDTA. The failure of some dogs to react in this manner is not explained. Possible mechanisms for decreased tubular reabsorption of phosphorus include stimulation of parathyroid secretion or effect of EDTA per se on tubular epithelium.

\section{Further Studies in Electrolyte and Water Metabolism in} Cardiac Patients Without Peripheral Edema. W. HoLLANDER and A. V. Chobanian, Boston, Mass. (Introduced by Francis C. Lowell).

Previous studies have shown that renal capacity to excrete sodium is not necessarily impaired in congestive heart failure. The present study was undertaken to determine whether any alteration in electrolyte and water metabolism could be detected by radioisotope dilution techniques in heart failure prior to an impairment of renal excretory capacity.

Twelve cardiac patients with symptoms but no striking signs of congestive heart failure were studied. Most of them (but not all) had a reduced cardiac output, a prolonged circulation time, or a slight elevation of venous pressure. The exchangeable body sodium ( $\mathrm{Na}^{\mathbf{*}}$ space) and extracellular fluid volume $\left(\mathrm{S}^{\infty} \mathrm{O}\right.$, space), as related to body weight, were significantly increased in this group whereas the exchangeable body potassium ( $\mathrm{K}^{\text {sa }}$ space) was normal as compared with 15 control subjects. Even though their total body sodium and extracellular fluid volume were increased, the cardiac patients were able to tolerate a daily 10-gram salt diet without developing a change in symptoms, an increase in weight, or retention of sodium. Furthermore, when rapidly infused with $300 \mathrm{cc}$. of 5 per cent sodium chloride, they excreted significantly more, rather than less, sodium than the control subjects even though their renal plasma flow (PAH clearance) and glomerular filtration rates (inulin clearance) were significantly reduced.

It was concluded that: 1 . An increase in body sodium and extracellular fluid volume occurs in congestive failure prior to impairment of renal capacity to excrete sodium. 2. Early in congestive heart failure a new equilibrium of expanded extracellular fluid volume apparently is established under which sodium and water excretion are homeostatically controlled.

\section{Pulmonary and Cardiac Blood Volumes of Patients With and Without Heart Disease. Rex L. HUfF,* DanIEL Parrish, and Wayne Crockett, Seattle, Wash.}

The amount of blood in the lungs, heart, and vessels between the lungs and heart was found to be 15.8 per cent of the total blood volume in recumbent patients without heart disease; when the formula, $V=F\left(\bar{T}_{2}-\bar{T}_{1}\right),(V=$ Volume, $F=$ Cardiac Output, and $\bar{T}_{2}$ is the mean transit time recorded at a point distal to the mean transit time, $\bar{T}_{1}$, at another point), was applied to data curves obtained by simultaneous recording of $\mathrm{I}^{120}$ HSA transients, detected with well-collimated crystals placed over the anterior thoracic wall. The values obtained in this manner are much less than those of more difficult clinically applicable techniques, and approach those obtained in animals by direct volume measurement after appropriate simultaneous double ligations. About 0.65 of the blood in the heart and lungs exists between the pulmonary capillaries and aorta. In patients with essential hypertension this value may exceed 0.80 in the absence of congestive heart failure. The time-radioactivity curves over the lung are nearly always "pure" as are those from over the left ventricle; however, those curves from the regions of the right atrium and pulmonary artery are occasionally compound. Nevertheless, extrapolation to form curves representing single chambers or channels is readily performed. Cardiac output values calculated from the in vivo data are nearly identical from each of the four locations. With the appropriate placement of detectors the location as well as amount of abnormal accumulation of blood may be ascertained. This technique, requiring only a single intravenous injection and two venous samples, gives much information of value in understanding and managing cardiac disorders. 
Gallop Rhythm in Mitral Valve Disease. Herberr HultGREN and Thomas Leo, San Francisco, Calif. (Introduced by David Rytand).

Since gallop rhythm is favored by rapid ventricular filling, gallop sounds should be rare in tight mitral stenosis and frequent in mitral insufficiency. The presence of a gallop may therefore indicate a mitral valve unsuitable for valvulotomy. To examine this possibility, a phonocardiographic study has been made of 22 patients with tight mitral stenosis, 12 patients with combined stenosis and insufficiency, and 4 patients with pure mitral insufficiency. All diagnoses were established at surgery, necropsy or both. Patients were adults, and none had acute carditis. A Sanborn Twin Beam phonocardiograph was employed, and simultaneous electrocardiograms, apex impulses and carotid pulses were recorded for timing purposes. The following observations were made:

1. No patients with tight mitral stenosis exhibited gallop sounds. In 3 patients with a mild degree of stenosis faint third sounds could be identified.

2. All patients with combined stenosis and insufficiency had prominent early diastolic gallops initiating short diastolic murmurs. The gallops were preceded by outward precordial motion which was swift when the gallops were loud, and slower when gallops were faint. The gallop sounds were often difficult to identify clinically because of their variable intensity, their low frequency and their proximity to the diastolic murmur. Two of these patients also had presystolic gallops. Absence of pulmonary hypertension and maximal intensity at the apex indicated a left ventricular origin of these presystolic sounds which may be mistaken clinically for presystolic murmurs.

3. All patients with pure mitral insufficiency had early diastolic gallops, but diastolic murmurs were faint or absent.

Left ventricular gallop sounds, because of their absence in tight mitral stenosis and presence in combined lesions and pure mitral insufficiency, are an important sign of significant mitral regurgitation. Since accurate identification by auscultation may be difficult, phonocardiography should be employed when mitral insufficiency is suspected.

Evidence for an Accessory Pathway of Galactose Metabolism in Man. Kurt J. Isselbacher, Boston, Mass. (Introduced by Mandel E. Cohen).

It has been demonstrated that the disease galactosemia is due to the congenital absence of a specific enzyme involved in the conversion of galactose to glucose. This enzyme, P-Gal transferase, is known to catalyze the following reaction:

(I) Gal-1-phosphate + Uridine diphosphate glucose (UDPGlucose) $\rightleftharpoons$ UDPGalactose + Glucose-1-phosphate

However, recent studies with $C^{16}$-galactose in an adult patient with galactosemia have shown conclusively that some conversion of galactose to glucose does occur in this disease in spite of the known enzymatic defect. This suggested that in all likelihood there must exist another pathway for forming UDPGalactose than that indicated by reaction I. Evidence has now been obtained by means of a cell-free preparation of mammalian liver and the use of $\mathrm{C}^{16}$-galactose-1-phosphate that there exists another route for the synthesis of UDPGalactose. The reaction has been shown to involve uridine triphosphate (UTP) and to proceed according to the following equation:

(II) Galactose-1-phosphate + UTP $\rightleftharpoons$ UDPGalactose + PP (pyrophosphate)

The enzyme involved is found in the nuclei of liver cells and is called "UDPGalactose pyrophosphorylase." The UDPGalactose formed via this pathway is then converted to UDPGlucose and in turn to glucose-1-phosphate by means of reactions previously described.

Although normally the liver contains much less pyrophosphorylase than transferase activity, it would appear that the existence of this additional pathway (II) could effectively account for the metabolism of galactose which occurs in galactosemia. The overall significance of these two pathways in normal individuals remains to be elucidated.

\section{Characteristics of a Previously Unidentified Protein Pres- ent in Normal Rat Serum. RALPH F. JACox,* Rochester, N. Y.}

Rat sera were separated electrophoretically utilizing a starch block technique. Electrophoresis was made for a period of 20 hours in $0.05 \mathrm{M}$ barbital buffer at $\mathrm{pH} 8.6$, with current of 450 volts and 20 milliamperes. Onecentimeter sections of the starch block were then eluted with $0.08 \mathrm{M}$ sodium chloride solution. Each eluate obtained was sub-fractionated by a cationic detergent technique (Jacox, R. F., J. Clin. Invest., 1953, 32, 661) to determine the distribution of components reacting with cationic detergent as alpha globulins, beta-gamma globulins and albumin. By these methods, a previously undescribed serum protein component has been found which has the following characteristics: 1) The new serum fraction migrates more rapidly than albumin and causes an asymmetrical appearance to the albumin peak. 2) It precipitates with 48 per cent saturation of $\left(\mathrm{NH}_{4}\right)_{2} \mathrm{SO}_{4}$ and thus has characteristics of a globulin; by this criterion it is not a mucoprotein. 3) The rapidly migrating "globulin" is present in serum in nearly the same concentration as albumin. 4) It reacts with cationic detergent at $\mathrm{pH} 6.65$, whereas albumin will not precipitate with cationic detergent at $\mathrm{pH}$ 9.5.

Thus, this fastest migrating electrophoretic component of rat serum has many characteristics of an alpha globulin.

Normal rats were given 20 mgms. of cortisone subcutaneously each day for 14 days. The serum protein values were determined daily by the cationic detergent method. A striking fall of the "alpha globulin" reacting component and an increase of albumin were observed after seven days of cortisone administration. The sera of cor- 
tisone-treated rats were then analyzed by starch electrophoresis followed by sub-fractionation with cationic detergent. A decrease of the rapidly migrating "globulin" and an increase in albumin concentration were observed, suggesting that some interconversion of these components may occur during cortisone intoxication.

\section{Cellular and Extracellular Alterations in Heart Failure:} Studies During Diuresis. JoHn R. JAENIKe and ChrisTINE WATERHouse, * Rochester, N. Y.

The significance of cellular changes in water and electrolyte composition in congestive heart failure has been a controversial issue. This question is particularly pertinent in the evaluation of hyponatremia accompanying severe failure. For this reason detailed metabolic studies have been conducted during diuresis in patients with heart disease of varying severity, utilizing nitrogen, electrolyte, water, and caloric balances.

In all subjects studied, during either mercurial or spontaneous diuresis, chloride space contraction and total water loss agreed closely, indicating an insignificant loss of cell water. This observation was verified by six studies in which body water changes were measured directly by deuterium dilution, which correlated well with changes calculated from water and caloric balance.

Significant differences in electrolyte metabolism were observed between various patients, however. Subjects readily responsive to mercurials or undergoing spontaneous diuresis excreted primarily sodium chloride, showed negligible internal shifts of sodium, and variable changes in potassium balance, probably unrelated to the heart failure per se. All patients in this group showed a calculated decrease in osmotically active total body cation which either equalled or slightly exceeded external losses, the latter suggesting osmotic inactivation of cation.

Successful mercurial diuresis was accomplished in three patients with advanced cardiac disease, previously refractory to therapy. Diuresis was characterized by relatively large urinary losses of potassium, low urinary sodium to chloride ratios, a shift of sodium into cells, and correction of pre-existent hyponatremia in two subjects. Clinical improvement ensued in the hyponatremic patients, and restoration of normal serum sodium concentration was attributable to excretion of extracellular water in excess of sodium, rather than an increase in extracellular fluid sodium content.

In contrast to other patients, diuresis in the hyponatremic subjects was accompanied by changes which suggested mobilization of osmotically active cation or correction of a deranged osmotic equilibrium between cells and extracellular fluid.

The Effect of Nucleosides on Osmotic Resistance of Mammalian Erythrocytes in Relation to the Age of the Cells. ERnst R. Jaffe, Grace A. Vanderhoff, BerTRAM A. Lowy, and Irving M. London,* New York, N. Y.

In studies on the relationship of metabolic activity of the erythrocyte to its structural integrity, the resistance of fresh human erythrocytes to osmotic stress has been found to be enhanced by incubation of the erythrocytes with various purine ribosides. The following experiments were designed to determine the influence of the age of the mammalian erythrocyte upon this effect of purine ribosides.

Whole blood from a normal adult man was collected in acid-citrate dextrose solution and stored at $4^{\circ} \mathrm{C}$. At intervals during the subsequent 168 days, aliquots of the stored erythrocytes were washed with isotonic sodium phosphate buffer, $\mathrm{pH}$ 7.3, and incubated in the buffer with and without nucleosides or glucose. After incubation the osmotic fragility was determined. The effect of adenosine or inosine in enhancing resistance to osmotic lysis was most marked initially and declined progressively; after 168 days no effect was demonstrable. The protective effects of glucose and deoxyadenosine were minimal.

Adult male rabbits received glycine-2- $\mathrm{C}^{1 /}$ for labeling of newly formed erythrocytes. At intervals of 4, 8, 13, 46 , and 60 days after injection of the labeled glycine, one of the rabbits was exsanguinated and the washed erythrocytes were incubated in isotonic sodium phosphate buffer, $\mathrm{pH}$ 7.3, with and without added nucleosides. After exposure to hypotonic phosphate buffer solutions, the extent of hemolysis and the radioactivity of the hemin isolated from the hemoglobin of the lysed cells were determined. It was found that young rabbit erythrocytes are more resistant to osmotic stress than older cells and that the effect of inosine in enhancing resistance to osmotic lysis is much more pronounced in young than in old erythrocytes.

The finding that the ability of purine ribosides to enhance osmotic resistance is more pronounced in young than in old erythrocytes is pertinent to the problems of erythrocyte preservation and of the mechanism of aging of erythrocytes in vivo.

\section{Serum Atropinase. B. V. JAGER,* Salt Lake City, Utah.}

Atropinase (tropinesterase), an enzyme present in the serum of certain rabbits, hydrolyzes atropine into pharmacologically inert tropine and tropic acid. The amount of enzyme present in the serum is determined by a manometric procedure, using atropine sulfate as a substrate. A related enzyme producing hydrolysis of atropine is found in the livers of many kinds of animals. In the present study, serum obtained from a wide variety of animal species was examined for the presence or absence of serum atropinase. Some types of rodents other than rabbits were found to have this enzyme in the serum. Striking differences were noted when the effects of atropine were compared in rabbits which had serum atropinase and in rabbits without this enzyme. This has lead to the development of a simple technic for detecting the presence or absence of atropinase in the serum of rabbits. This has been examined in over 100 rabbits. 
Vitamin $B_{10}$ Activity of Leukemic Sera and Tissues. G. Watson JAMES, III * and Lors RIskin, Richmond, Va.

The unicellular chrysomonad, Poteriochromonas stipitata, like $O$. malhemensis is considered to have a specific growth requirement for cyanocobalamin and differs in this respect from Euglena gracilis, the usual assay organism. In 30 normal individuals the vitamin $B_{12}$ activity of the serum assayed 0 to $275 \mu \mu \mathrm{g}$. per ml. (mean $=44 \mu \mu \mathrm{g}$. per ml., standard error of mean $=11) ; 15$ patients with chronic granulocytic leukemia had levels of 100 to $11,750 \mu \mu \mathrm{g}$. per ml. (mean $=1676 \mu \mu \mathrm{g}$. per ml., standard error of mean $=194)$; 14 patients with acute leukemia (not terminal) assayed from 0 to $132 \mu \mu \mathrm{g}$. per ml. (mean $=29 \mu \mu \mathrm{g}$. per ml. with standard error of mean $=4)$. Five patients with untreated pernicious anemia had less than $1 \mu \mu \mathrm{g}$. per ml. vitamin $B_{12}$ activity. Patients with various hematologic disorders and those with pernicious anemia under treatment were not appreciably different from normal individuals.

In the same sera, comparison of two assay methods demonstrated in ten normal subjects that the Euglena gracilis assay (304 to $728 \mu \mu \mathrm{g}$. per ml., mean $=455 \mu \mu \mathrm{g}$. per ml.) was six times greater than that for P. stipitata; however, in nine chronic granulocytic leukemia patients the Euglena assay (370 to $11,533 \mu \mu \mathrm{g}$. per ml., mean $=3655 \mu \mu \mathrm{g}$. per ml.) was roughly twice that of the $P$. stipitata. Other hematologic disorders were similar to the normals. When leukemic sera and normal sera are assayed with $P$. stipitata using amounts of extract representing $0.05,0.1,0.2$, and $0.4 \mathrm{ml}$. of serum a striking difference is observed. Normal sera, sera from acute leukemia patients and subjects with various hematologic disorders showed no significant difference in total calculated values ( $\mu \mu \mathrm{g}$. per ml.) at the four different serum levels, whereas in the leukemic sera the assay value increased with increasing amounts of serum.

Vitamin $B_{12}$ activity of liver, kidney and spleen from leukemia patients and normal individuals decreased as the amount of tissue content increased suggesting the presence of tissue inhibitor substance to the growth of P. stipitata.

Pyrogenic and Leukopenic Effects of Immune Hemolysis in Man. James H. JANDL, Boston, Mass. (Introduced by W. B. Castle).

Chills, fever, and alterations of leukocyte levels are frequently observed during episodes of hemolysis. Injections of small amounts of washed, $\mathrm{Cr}^{\mathrm{G}}$-labelled $\mathrm{ABO}$ incompatible red cells into normal subjects caused an abrupt, profound fall in circulating granulocytes and monocytes, coinciding with the sequestration of the labelled red cells and the onset of hemoglobinemia. Within 20 minutes an immature granulocytosis appeared, followed by an adult granulocytosis, lymphopenia, and occasionally mild fever (average rise: $0.5^{\circ} \mathrm{F}$ ). Comparable preparations of type D red cells injected into D-sensitized subjects caused a similarly profound but more slowly de- veloping and more prolonged leukopenia, which again coincided in onset with the slower rate of red cell destruction. In these subjects marked pyrogenic responses occurred invariably, consisting of initial hypothermia, followed by chills appearing at the time of extreme leukopenia, and then fever (average rise: $3.0^{\circ} \mathrm{F}$ ) associated with an immature granulocytosis and lymphopenia. Injection into normal subjects of type $D$ red cells sensitized in vitro with incomplete anti-D serum produced almost identical phenomena. Neither fever nor leukopenia followed injections of hemoglobin, whole blood hemolysates, or soluble red cell antigens, or the destruction of red cells in vivo by non-immune mechanisms.

In vitro, anti-D sensitized red cells, unlike normal cells, were observed to cluster tightly around individual granulocytes, particularly those of immunized subjects, and upon adhering, to become spherocytes.

These studies indicate that the sequestration of red cells by immune mechanisms in man is attended by leukopenia (leukocyte destruction?) and the release of "endogenous pyrogen." The effects of red cell destruction by incomplete and by complete antibodies, respectively, are analogous to the effects described by others of bacterial pyrogens on normal and "tolerant" animals. While being themselves destroyed, granulocytes are seemingly involved in the spherocytosis and hemolysis of red cells altered by antibodies in vivo.

\section{Mechanisms of Pancreatic Secretion of Electrolytes in Man: The Effects of Diamox and Disease. Henry D. Janowitz* and David A. Dreiling, New York, N. Y.}

The relationship between rate of flow and electrolyte composition of pancreatic juice stimulated by secretin or inhibited by Diamox ${ }^{\text {(B) }}$ was determined in 65 normal subjects during continuous aspiration of duodenal contents free of gastric contamination. In these individuals, $\mathrm{HCO}_{3}$ concentration (25 to $151 \mathrm{mEq}$. per L.) varied directly with rate of secretion, $\mathrm{Cl}$ varied inversely, and the sum $\left(\mathrm{HCO}_{3}+\mathrm{Cl}\right.$ ) remained constant (155 to $157 \mathrm{mEq}$. per L.), approaching the osmolarity of the plasma. Pancreatic excretion of $\mathrm{Na}$ (139 to $143 \mathrm{mEq}$. per L.) and $\mathrm{K}$ ( 6 to $9 \mathrm{mEq}$. per L.) was independent of the rate of flow. When the rate of secretion was inhibited by i.v. administered Diamox (50 to $121 \mathrm{mg}$. per $\mathrm{Kg}$. body weight) in 14 normal subjects, the maximum $\mathrm{HCO}_{2}$ concentration tended to remain constant despite marked reduction of volume flow (mean inhibition $=69$ per cent) and $\mathrm{HCO}_{3}$ output (mean inhibition $=72$ per cent). These changes were independent of plasma electrolyte alterations. The concentrations of $\mathrm{Na}, \mathrm{K}$, and the sum $\left(\mathrm{HCO}_{s}+\mathrm{Cl}\right)$ were unaltered.

These relationships held for 61 patients with pancreatitis except that they were unable to achieve as high $\mathrm{HCO}_{3}$ concentrations at comparable rates of flow (max. 81 mEq. per L.).

Previous investigators have hypothesized that the elec- 
trolyte composition of pancreatic juice at varying rates of flow represents the admixture of an isotonic $\mathrm{HCO}_{3}$ solution with one resembling interstitial fluid. The present observations indicate that carbonic anhydrase activity is required for the elaboration of the major portion of the $\mathrm{HCO}_{\mathrm{s}}$ output of the human pancreas. They also suggest that the $\mathrm{HCO}_{8} / \mathrm{Cl}$ ratio results from alteration of the primary secretion of isotonic $\mathrm{HCO}_{3}$ by a process of limited capacity in which $\mathrm{HCO}_{3}$ is exchanged for $\mathrm{Cl}$, presumed to occur in the intercalated ducts, and that Diamox interferes with this exchange.

\section{Etiology of Epidemic Keratoconjunctivitis. ERNEST} Jawetz,* Yukiniko Mitsui, Lavelle Hanna, Phillips Thygeson, and Samuel Kimura, San Francisco, Calif. and Kumamoto, Japan.

Epidemic keratoconjunctivitis (EKC) is a serious eye disease of well defined clinical characteristics. Following an acute inflammatory stage there develop round subepithelial opacities in the cornea which may persist up to two years and seriously interfere with vision. EKC occurs both sporadically and in epidemics all over the world. Its etiology is not established. Claims have been made for the role played by different viruses but most of them have not been substantiated. Textbooks list a virus (Sanders) resembling St. Louis encephalitis virus as etiologic agent. The available strains of this virus bear no serological relationship to the disease as it has existed since 1951. Based on the evidence presented below it is suggested that adenovirus type 8 (APC 8) is regularly associated with EKC and is capable of causing this disease.

1. Six strains of APC 8 have been isolated to date, all from eyes, five of which were clinically definite EKC. No strains of this virus have been isolated from other sources. APC 8 differs biologically from other adenovirus types.

2. Among 147 patients diagnosed as EKC during 1951-56 in North America, Japan, Austria, Germany, Switzerland, and Italy, 115 (78.2 per cent) had neutralizing antibodies to APC 8 in a serum dilution of $1: 10$ or greater. Among 174 controls from the same geographic areas and similar age distribution only 13 (7.4 per cent) had such antibody titers.

3. Of 25 adequate paired sera from patients diagnosed EKC, 21 showed a four-fold or greater rise in neutralizing antibodies to APC 8 but not to other adenovirus types. APC 8 antibodies declined rapidly so that titers of 1:20 were not found later than two years after onset.

4. One eye of each of five volunteers was inoculated with APC 8. Four developed typical EKC; the fifth had APC 8 antibodies at the time of inoculation and remained well.

The uninoculated eye became involved in three volunteers six to eight days after the inoculated eye. APC 8 virus was re-isolated from lesions and the volunteers developed significant antibody titer rises.
The Response of Diffuse Hyperfunctioning Goiter to Thyrotropin. W. McK. JefFeries, R. P. Levy, L. W. KeILY, JR., and G. Cooper, Cleveland, O. (Introduced by W. H. Pritchard).

The change in 3-hour thyroidal $\mathrm{I}^{181}$ uptake and serum protein-bound iodine (PBI) before and 24 hours after an intramuscular injection of thyrotropin (TSH) has been shown to reflect the effect of this hormone upon rate of iodide uptake and rate of hormonal release, respectively. In order to learn more about the pathologic physiology of hyperthyroidism with diffuse goiter, the responses of patients with this disease to a single injection of TSH before and at intervals after treatment have been studied. Of 19 subjects tested before treatment, 6 had rises in either $\mathrm{I}^{181}$ uptake or PBI or both of a degree which was probably significant (an increase of over 15 per cent of the tracer in the 3-hour uptake and over $3 \mathrm{mcg}$. per $100 \mathrm{ml}$., respectively). The remainder showed no response. These findings indicate that the thyroids of at least some persons with this disease do not continuously function at a maximum rate. Furthermore, they reveal that some hyperfunctioning thyroids may be trapping iodide at a maximum rate without releasing hormone at a maximum rate, whereas others may be releasing hormone as rapidly as it is formed even though iodide is not being trapped at a maximum rate. Neither severity of symptoms nor presence of exophthalmos correlated well with the rate of $I^{13}$ uptake, the level of PBI, or the type of response to TSH. Tests on 10 patients with recurrent Graves' Disease revealed a similar distribution of responses.

Of the apparently euthyroid patients tested up to 34 years after subtotal thyroidectomy and up to 5 years after $\mathrm{I}^{181}$ therapy, most showed evidence that the remaining thyroid tissue continued to function at a maximum rate, although a few had normal responses. Some patients after treatment for hyperthyroidism had normal $\mathrm{I}^{121}$ uptakes with low PBI's or elevated uptakes with normal PBI's, demonstrating that $I^{28}$ uptake is not a reliable indication of the state of thyroid function in such circumstances.

Arterial Oxygen Unsaturation and Possible Mechanisms of Its Production in Sickle Cell Anemia. Wallace N. Jensen, Donald L. Rucknager, and W. Jape Taylor, Pittsburgh, $\mathrm{Pa}$. (Introduced by I. Arthur Mirsky).

Oxygen unsaturation of arterial blood has been found in a majority of patients with sickle cell anemia, in contrast to the normal oxygen saturation found in other types of anemia. In 19 determinations of arterial oxygen saturation, performed at widely spaced time intervals in 11 patients with sickle cell anemia, the range of saturation was 72 to 97 per cent with a mean of $85 \pm 7.1$ per cent. There was no correlation between the degree of anemia and the arterial oxygen unsaturation. One hundred per cent oxygen was administered by mask to 7 patients with a prompt rise in arterial oxygen saturation to normal levels. The infusion of normal blood to 
5 patients in amounts sufficient to produce a mean increment of $3.7 \mathrm{gm}$. per cent hemoglobin was associated with a mean rise in oxygen saturation from a pre-transfusion mean of 83 per cent to a post-transfusion mean level of 93 per cent $(p<0.01)$.

The complete arterial oxygen saturation observed with 100 per cent oxygen breathing indicates that the arterial oxygen unsaturation is not the result of a circulatory veno-arterial shunt. The increased arterial oxygen saturation following the transfusion of normal erythrocytes indicates that diffuse pulmonary disease is not, alone, sufficient to cause the unsaturation. Since arterial oxygen unsaturation does not exist in other uncomplicated types of anemia, an increased rate of blood flow through the lungs in sickle cell anemia cannot, per se, explain the arterial oxygen unsaturation. It appears that the combination of a short pulmonary circulation time plus either diffuse subclinical pulmonary disease or an erythrocytic defect in the mechanism for oxygen uptake in sickle anemia cells provides the basis for arterial unsaturation.

\section{The Contractility of Actomyosin Bands Prepared from}

Human Heart Muscle. K. Kako and G. CABrera, St. Louis, Mo. (Introduced by R. J. Bing).

Previously published studies of the afterloaded contractions of acetomyosin bands prepared from dogs' hearts have revealed no difference between the fresh preparation and that obtained one hour after death. This finding suggested the possibility of studying the contractile properties of actomyosin prepared from human hearts obtained during post-mortem examination. Actomyosin was prepared from human hearts obtained from one to four hours following the death of the patient. The protein was extracted in the usual manner. Actomyosin bands were prepared in a trough by compression of surface spread films of the protein. ATP was added and contractions were recorded with a null seeking device with the bands swimming on the surface of the trough solution. All contractions were afterloaded and shortening of the bands was expressed as a function of the load per "specific nitrogen content" of the bands. The latter represents the amount of nitrogen in micrograms per one millimeter of band in resting length. The results indicate that actomyosin bands prepared from human heart muscle shortened from 20 to 40 per cent of their initial length. No difference in shortening of fibers prepared one to four hours after death of the patient was noticeable, and the addition of a cardiac glycoside (digoxin) had no effect on the shortening of the bands. The contractility of actomyosin prepared from human hearts in failure will be discussed.

The Effects of Muscular Exercise on Net Splanchnic Glucose Production (NSGP) in Man. RoBker F. Kibler and W. Jape Taylor, Pittsburgh, Pa. (Introduced by Jonas E. Salk).

In the resting, fasting state both the arterial glucose level and the hepatic glucose production are relatively constant. It has been estimated that under these circumstances approximately 80 per cent of the hepatic glucose output is utilized by the brain and the remaining 20 per cent by other tissues, presumably muscle. A1though never proven, it might be assumed that muscular work would be accompanied by an increased hepatic glucose production. Accordingly, the effect of moderate to severe exercise on the fasting NSGP has been studied in six control, hospital subjects.

Exercise was accomplished in the recumbent position by pedaling a bicycle ergometer. A constant level of exercise was performed by four subjects for 15 to 40 minutes, in three of whom the total oxygen consumption increased by 140 per cent. The two remaining subjects exercised at an increasing rate to the point of exhaustion which occurred at 30 and 40 minutes. NSGP was determined as the product of the BSP hepatic blood flow and hepatic venous-arterial glucose difference. Measurements were made at five-minute intervals during exercise and in one subject for a twenty-minute period following exercise.

At no time during exercise did the NSGP change significantly from the control value. Arterial blood glucose levels did not fall in three subjects and in the other three fell between 3 and $6 \mathrm{mgm}$. per cent. The hepatic blood flow, BSP clearance and splanchnic oxygen consumption showed no significant change.

From these data, it is concluded that whatever additional glucose the muscles utilize during exercise of the degree performed in these studies is derived from muscle stores alone; and that during exercise the brain remains the major consumer of the hepatic glucose production. Studies are in progress to obtain confirmatory evidence for this conclusion from measurements of femoral and cerebral arteriovenous glucose differences during exercise.

Effect of Mycobacteriophage on Mycobacteria In Vivo. Vernon KNIGH ${ }^{*}$ and Arthur White, Nashville, Tenn.

We have observed that a mycobacterial bacteriophage, $\mathrm{D}-29$, isolated by others, causes lysis of several strains of human tuberculosis as well as the non-virulent $M$. tuberculosis, ATCC 607 . It was further found that homogenate of guinea pig spleen did not inactivate the phage in vitro and that phage propagation occurred in high concentrations of human serum. Moreover, active phage could be recovered as long as 24 hours following injection into mice. Possible effects of phage on susceptible bacteria are (1) induction of lysogenesis, (2) development of phage resistance without lysogenesis, or (3) destruction of bacterial inocula by phage lysis. Studies with diphtheria bacilli have shown that the induction of lysogenesis may be accompanied by development of toxigenicity (virulence) of a previously avirulent culture. From studies with choleraphage it appears that changes in virulence of the vibrio may occur in the course of natural infection, as a consequence of phage activity. Therefore, in the absence of known inactivating host 
mechanisms, experiments were performed to examine the effect of mycobacteriophage on mycobacteria, in vivo. Mice were inoculated intravenously with cultures of $M$.

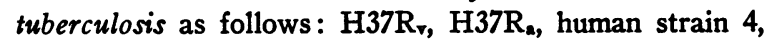
ATCC 607, and phage resistant ATCC 607. Several schedules of parenteral phage dosage were employed and the course of infection was followed by observation of mortality rates, or by colony counts of splenic homogenates obtained at intervals during the infection. Studies of myobacteria recovered from treated animals did not reveal evidence of lysogenesis, new development of phage resistance, nor modification of infection by phage treatment. The originally phage resistant cultures of ATCC 607 retained this characteristic through animal passage. The implications of these studies will be discussed.

A Six-Month Evaluation of an Anabolic Drug, Norethandrolone, in Chronically Underweight Individuals. Ross C. Kory, Robert N. Watson, Matthew H. Bradley, and Bruno J. Peters, Milwaukee, Wisc. (Introduced by William W. Engstrom).

Norethandrolone, $17 \alpha$-ethyl-17-hydroxy-norandrostenone (Nilevar ${ }^{(8)}$ ), has been shown to be an effective anabolic agent in patients with acute illnesses. The present study is a long-term evaluation of this steroid in underweight but essentially healthy individuals.

A daily oral tablet containing either placebo, $25 \mathrm{mg}$., or $50 \mathrm{mg}$. of norethandrolone was administered by the "double blind" technique to 56 underweight individuals for three months. These included: a) 29 young factory workers, 11 female and 18 male; b) 17 elderly male members of a domiciliary unit; and c) 10 male hospitalized patients with stabilized pulmonary tuberculosis. The code was then broken, the placebos discontinued, and all were given the steroid for three additional months.

Statistically significant gains in weight were observed in all groups receiving norethandrolone $(p<0.01)$; these usually were accompanied by increases in appetite and sense of well-being. The average weight gain was two pounds per month. The maximum was 20 pounds in six months. No patient exhibited edema or other signs of fluid retention.

Undesirable clinical effects were not observed in the male patients but appeared in nine of the eleven females; and consisted of menorrhagia in two, acne in four, and amenorrhea in seven. These effects were reversed by reducing the dosage or discontinuing the drug.

Unexpected retention of bromsulfalein was observed in about three-fourths of the patients. Abnormal dye retention was considerable (up to 51 per cent), other liver function tests were entirely normal, no clinical signs of liver disease appeared, and the effect was reversible with discontinuance of the drug. Liver biopsies in six individuals with high dye retention were normal. While a relationship may exist, no correlation could be made between weight gain and dye retention. These observations require explanation and the long-term use of steroids of this nature carefully appraised.
Factors in the Genesis of Pulsus Bisferiens. PETER T. Kuo and Arthur F. Whereat, Philadelphia, Pa. (Introduced by William A. Jeffers).

In a patient with luetic aortitis, aortic insufficiency and dissecting aneurysm of the aorta, a bifid pulse was found that was limited to the left arm only. This pulse pattern appeared as a catheter was withdrawn from the aorta to a point just distal to the dissection. This led to the study of 14 other patients with pulsus bisferiens who suffered from a variety of conditions, including : aortic valvular disease, patent ductus arteriosus, and aneurysm of the sinus of Valsalva that had ruptured into the right ventricle. Arterial and left ventricular pressure curves of these patients were obtained by arterial and retrograde left ventricular catheterization, using a small plastic catheter inserted percutaneously through a thin-walled 20 or 21-gauge needle. In all these 14 patients a bifid pulse was recorded in both brachial vessels, while a curve with many high frequency vibrations was simultaneously recorded in the femoral or the carotid artery. Exploration of the arterial tree indicated that the subclavianbrachial system was particularly prone to develop a bisferious pulse, and that aortic stenosis per se contributed little or nothing to the production of this pulse pattern. In all the patients pulsus bisferiens was increased by exercise, epinephrine or nor-epinephrine infusion, or by mechanical compression of a peripheral artery. In all it was decreased, after a premature beat, during the Valsalva maneuver, by amyl nitrite inhalation, and by the onset of severe heart failure. It is concluded that pulsus bisferiens is primarily a peripheral manifestation of an increase in cardiac stroke volume and velocity of peripheral blood flow.

\section{The Pharmacology of the Placebo. Lours Lasagna,* Baltimore, Md.}

It is well known that placebos can mimic drugs in producing therapeutic benefit or untoward effects. Certain details of the pharmacology of placebos are less widely appreciated. Data collected in experiments on a variety of populations (134 post-partum patients, 34 tuberculous patients in a sanatorium, 31 patients in a chronic disease hospital, and 93 patients suffering from post-operative pain) indicate how closely placebo effects may at times resemble effects generally considered attributable only to "active" agents.

These phenomena include: 1) time-effect relationships, both in acute single dose experiments and in repeated daily administration of placebos, and 2) correlation between efficacy of placebos and severity of disease. In single dose experiments, for example, "peaking" of therapeutic effect can be observed with placebos, with an initial building up, and a subsequent waning, of effect over a period of hours. "Cumulative" effects are likewise seen with placebos, in that progressive increase in therapeutic benefit may occur with repeated daily doses. "Carry-over" effects have also occurred, with therapeutic effect persisting for variable periods of time after cessation of 
placebo therapy. In treating post-operative and postpartum pain, morphine, aspirin, and placebo all appear less effective in relieving severe pain than in relieving mild pain. Thus with placebos, as with active drugs, one can show a negative correlation between severity of complaint and therapeutic efficacy.

The implications of these data for the interpretation of uncontrolled experiments and for the design of controlled experiments will be discussed.

Uric Acid Formation in Patients with Gout. The Incorporation of 4-amino-5-imidazolecarboxamide- $6^{13}$ into Uric Acid. Leonard Laster, J. E. Seegmiller, DeWitt Stetren, JR., and LoIs V. Liddee, Bethesda, Md. (Introduced by John T. King).

From studies of the incorporation of glycine- $\mathrm{N}^{15}$ into uric acid, Benedict et al. concluded that some gouty patients may possess a "shunt pathway" for urate biosynthesis. We have studied the incorporation of the purine precursor 4-amino-5-imidazolecarboxamide-C ${ }^{18}$, "AIC," into uric acid in normal and gouty persons in order to explore further the nature of the proposed "shunt."

In the normal human, fed AIC-C ${ }^{13}$ is incorporated more promptly into urinary uric acid and in larger amounts than is glycine- $\mathrm{N}^{15}$, and in a pattern that implicates a rapid and slow conversion. AIC depresses the incorporation into uric acid of simultaneously administered glycine- $\mathrm{N}^{15}$ without affecting its incorporation into creatinine, suggesting that there is a regulatory mechanism for endogenous urate production.

In five gouty patients studied so far the extent of AIC-C ${ }^{18}$ incorporation into uric acid was greater than normal and was substantially greater in those patients who showed an excessive incorporation of glycine- $\mathrm{N}^{15}$ into uric acid. In all the patients the conversion of AIC-C ${ }^{13}$ to uric acid occurred by rapid and slow pathways. In addition AIC-C $\mathrm{C}^{18}$ suppressed the incorporation of glycine$\mathrm{N}^{15}$ fed simultaneously. Although the per cent suppression varied considerably in different gouty subjects, two of them still incorporated into uric acid about twice as much $\mathrm{N}^{15}$ from glycine as did normal subjects under similar conditions of AIC administration.

These results suggest that there may be a defective regulatory mechanism for endogenous purine synthesis in some gouty patients.

The Renal Transport of Hemoglobin in Renal Disease. Willoughby Lathem, Pittsburgh, Pa. (Introduced by Jack D. Myers).

Physiologic and pathologic evidence suggests that the transport of protein molecules across both the glomerular membrane and renal tubules may be altered in renal disease. However, quantitative information regarding these processes is limited. In the present study the rate of transfer of hemoglobin molecules at these sites was measured in patients with a variety of chronic renal diseases and proteinuria.

Approximately $10 \mathrm{gm}$. of hemoglobin (4 per cent solution prepared by hemolyzing the subject's erythrocytes in distilled water) were administered intravenously over a period of 30 minutes. Plasma and urinary hemoglobin concentrations were determined every 15 to 30 minutes for two hours. The clearance of inulin $\left(C_{1 n}\right)$ was measured concurrently.

A linear relationship between the rate of hemoglobin excretion $\left(U_{\text {hgb }} V\right)$ and the plasma level $\left(P_{h g b}\right)$ was obtained, indicating a constant tubular reabsorptive rate ( $T_{\text {hgb }}$ ) at the levels of $P_{\text {hgb }}$ examined (below $400 \mathrm{mgm}$. per cent). This permitted the following calculations:

Plasma cleared of hemoglobin at the glomerulus $\left(\mathrm{C}_{\mathrm{hgb}}\right)$

$$
=\frac{\Delta \mathrm{U}_{\mathrm{hgb}} \mathrm{V}}{\Delta \mathrm{P}_{\mathrm{hgb}}}(\mathrm{ml} \text {. per min.) }
$$

Transfer rate of hemoglobin across the glomerulus (load)

$$
=\frac{\Delta \mathrm{U}_{\text {hgb }} \mathrm{V}}{\Delta \mathrm{P}_{\text {hgb }}} \times \mathrm{P}_{\text {hgb }} \text { (mgm. per min.) }
$$

Tubular hemoglobin reabsorption $\left(\mathrm{T}_{\mathrm{hgb}}\right)$

$$
=\frac{\Delta \mathrm{U}_{\mathrm{hgb}} \mathrm{V}}{\Delta \mathrm{P}_{\mathrm{hgb}}} \times \mathrm{P}_{\mathrm{hgb}}-\mathrm{U}_{\mathrm{hgb}} \mathrm{V} \text { (mgm. per min.) }
$$

(The expression $\mathrm{Tm}_{\mathrm{hgb}}$ was not employed owing to uncertainty concerning glomerular activity and tubular saturation in diseased nephrons.)

In subjects with renal disease $\mathrm{C}_{\text {hgb }}$ averaged $3.4 \mathrm{ml}$. per min. (normal, 10.8); $\mathrm{T}_{\text {hgb }}$ averaged $5.0 \mathrm{ml}$. per min. (normal, 10.2) $; \mathrm{C}_{\text {hgb }} / \mathrm{C}_{\text {in }}$ averaged 0.034 (normal, 0.090); and $T_{\text {hgb }}$ per $100 \mathrm{ml}$. filtrate averaged $4.6 \mathrm{mgm}$. (normal, 8.9). These changes were not correlated with the degree of proteinuria or with the severity of renal functional impairment.

These results indicate that, at levels of $P_{\mathrm{hgb}}$ obtained, the transglomerular and tubular transport of hemoglobin is reduced in renal disease, both in absolute and relative (to $C_{1 n}$ ) terms. These changes may be attributable to structural or metabolic alterations in the diseased kidney, to competitive activity with plasma proteins, or to both.

Effect of Specific Antigen on Release from Human Leucocytes of the Factor Concerned in Transfer of Delayed Hypersensitivity. H. S. LAwrence * and A. M. PAPPENHEIMER, JR., New York, N. Y.

It has been shown that the delayed tuberculin type of hypersensitivity may be transferred to nonsensitive human recipients by injection of leucocytes or leucocyte extracts obtained from the blood of sensitive donors. The transfer factor (TF) resists treatment with $\mathrm{DN}$-ase, $\mathrm{RN}$-ase and crystalline trypsin. We are now reporting evidence that TF may be liberated from sensitive leucocytes by interaction with specific antigen.

One $\mathrm{ml}$. of packed leucocytes from a highly tuberculin sensitive donor was suspended in the donor's fresh serum (or plasma) and divided in two equal portions. Twentyfive gamma PPD were added to the test suspension; the other served as control. Both suspensions were aerated by gentle rotation in siliconized tubes for 1 hour at $37^{\circ} \mathrm{C}$. After centrifugation, the frozen and thawed DN-asetreated test and control cell extracts were injected into 
each of 2 tuberculin negative recipients. The corresponding supernates were injected into 2 other recipients. One week after transfer the recipients were skin tested with old tuberculin. The results showed that contact with PPD caused the complete extraction of TF into the supernate serum or plasma and the PPD-treated cells failed to effect transfer of tuberculin sensitivity. No TF could be detected in the control supernate.

The above experiment was repeated with identical results.

Donor H.C. was also sensitive to diphtheria toxoid. That treatment of cells with PPD caused specific release of the TF concerned with tuberculin sensitivity was shown by the fact that transfer of sensitivity to toxoid could only be effected by the PPD-treated cell residue.

No evidence could be obtained that PPD caused significant damage to any major fraction of the cells.

\section{Positive Response to Chorionic Gonadotrophin in Kline-} felter's Syndrome. RoBERT B. LEACH, WILLIAM O. Maddock,* C. Alvin Paulsen, John Lanman, and Warren O. Nelson, Detroit, Mich., and Iowa City, Ia.

Chorionic gonadotrophin stimulates the Leydig cells of normal men to secrete increased amounts of estrogen and androgen. Urinary estrogen excretion and clinical evidence of increased androgen secretion were taken as criteria of response in four patients having Klinefelter's syndrome who received chorionic gonadotrophin (APL) three times weekly for two weeks to seven months. The diagnosis of Klinefelter's syndrome was based on high urinary gonadotrophins, buccal smears or skin biopsies showing a female genetic sex pattern, and testicular biopsies revealing hyalinized seminiferous tubules and clumped Leydig cells.

During treatment, each patient showed a definite response. Urinary estrogen excretion increased from low to normal levels in two moderately eunuchoidal patients, R.W. and C.B., age 18 and 51 years. The younger patient experienced a marked increase in number of erections, penile growth, and a striking change to normal adolescent personality. The older patient, who was treated for only two weeks, showed no clinical response. Subsequently, a response to large doses of testosterone was noted only after a month of treatment. Patient J.B., age 27, was a normal appearing male. Potency was normal, and was not affected by treatment. Urinary estrogen excretion, normal before treatment, increased four fold by the third week of treatment. Patient H.T., age 15, had undescended testes and moderate puberal development. During therapy, the gonads descended to the external ring, erections increased in frequency, and penile growth was noted. Urinary estrogens were not determined.

It is concluded that the Leydig cells in Klinefelter's syndrome remain responsive to gonadotrophin stimulation. The chorionic gonadotrophin therapeutic test is not valid for separating hypo- and hyper-gonadotrophic types of eunuchoidism.
Effects of Prednisone on Glucose Tolerance in Liver Disease. Carroll M. Leevy, Jersey City, N. J. (Introduced by Harold Jeghers).

Compound $\mathrm{E}$ and its analogues alter glucose tolerance in normal subjects and produce hyperglycemia and glycosuria in the presence of latent diabetes. The influence of hepatic disease on encountered responses was studied in twenty-five patients with liver disease and a normal fasting blood sugar level. Eight patients had acute viral hepatitis, five fatty liver, and twelve moderately advanced cirrhosis. After patients had been on a 300-gram carbohydrate intake for three to five days, oral glucose tolerance tests were performed using 1.75 grams of glucose per kilogram of body weight. Twenty-four to forty-eight hours later, this test was repeated after giving 30 milligrams of prednisone in two divided doses, one and six hours prior to ingestion of glucose.

Results of the prednisone-glucose tolerance test were correlated with hepatic functional reserve. Glucose tolerance curves without prednisone were normal in eight patients, flat in seven, and diabetic in ten. Addition of prednisone induced a diabetic response in four patients with normal curves, converted a flat curve to a normal pattern in three patients, and increased the height of each of the diabetic curves. After improvement in hepatic function, prednisone-glucose tolerance tests were normal in three patients in whom diabetic curves were induced by prednisone, and in three patients in whom flat curves were not altered by steroids. Prednisone produced a diabetic curve after all evidence of liver disease had disappeared in two patients who subsequently developed clinical diabetes.

Citric Acid Metabolism in Bone and Joint Disease. Henry M. Lemon, Julius H. Mueller, III, William H. Chasen, and Joseph M. Looney, Boston, Mass. (Introduced by Chester S. Keefer)

Citric acid tolerance tests have been performed in 6 healthy individuals, 36 patients with osteoarthritis, rheumatoid arthritis of peripheral joints, and ankylosing spondylitis, and 24 patients with inoperable cancer. The latter included carcinomas of the breast, bronchus, pulmonary alveolus, thymus, ovary, endometrium, leiomyosarcoma and multiple myeloma. Venous serum citrate was determined using Ettinger's modification of the pentobromoacetone method (J. Biol. Chem., 1952, 199, 531) after overnight fasting, $5,10,20$, and 30 minutes following rapid I.V. injection of $0.5 \mathrm{gm}$. sodium citrate. A two-fold increase in the normal serum citrate concentration was thereby produced within 5 minutes, which, however, did not result in any significant change in serum calcium, phosphate, or glucose concentrations at $\mathbf{3 0}$ minutes.

The mean fasting citrate concentration of normals and arthritics was identical (27 to 29 gamma per ml. \pm 2 to 7 ), but that of cancer patients was significantly elevated $(43 \pm 21$; S.E. diff. $=4.5, t=3.25)$. The serum citrate response of cancer patients usually remained significantly 
elevated at all intervals throughout the test. In all groups serum citrate concentration after 30 minutes approached initial values, without variations in the rate of removal of citrate except in the cancer group, in whom slightly greater rates were noted.

Although bone contains the highest tissue concentration of citric acid ( 0.8 to 1.6 per cent), the fasting citrate level either may be elevated in the absence of roentgen evidence of bone destruction, or normal in patients with biopsied osseous metastases. Since all patients with elevated serum citrate levels also had increased copper resistant serum acid phosphatase (Reynolds et al., Cancer Research, 1956, 16, 943), it seems most likely that these observations reflect the metabolic activity of cancerous tissue which is relatively rich in citric acid compared to normal soft tissues.

The Mechanism of the Auricular Gallop. James J. LeoNARD, Arnold M. Weissler, and James V. Warren,* Durham, N. C.

The auricular (presystolic) gallop is a low frequency sound occurring after auricular systole and before subsequent ventricular contraction. With careful auscultation, this sound may be heard frequently in patients with hypertension, coronary artery disease, and first degree heart block.

Eighteen patients with readily audible auricular gallop rhythm were investigated. Blood pooling was effected by occlusive cuffs on all four extremities. Apex cardiograms and logarithmic phonocardiograms were recorded before, during and after inflation of these cuffs to diastolic levels for five minutes.

With cuff inflation, the gallop sound migrated towards the first heart sound, as did its equivalent wave on the apex cardiogram. Accompanying this movement was a striking diminution, or disappearance of the gallop in twelve cases. When the auricular vibrations did not completely disappear, they were seen as small, slow deflections blending with early components of the first heart sound. Upon release of cuffs, they moved back to their former position.

Auricular contraction produces a pressure wave in the ventricle, thought to cause tensing or even reclosure of the AV valve. The observations reported here suggest that under certain circumstances, transmission of this wave is accelerated. As a result, an auricular component of the first heart sound appears early and is split off from the remainder. If the separation is wide enough $(>0.04 \mathrm{sec}$.$) , the auricular sound is heard separately,$ creating a gallop rhythm. The hemodynamic effect of blood pooling returns the auricular sound components to a more normal position. At times separation may be enhanced by a delay in the appearance of the major vibrations of the first sound, evidenced by slight prolongation of the Q-1 time. In patients with heart block and auricular gallop, similar separation of an auricular sound component occurs because of the conduction delay between auricle and ventricle.
The Genesis of Hypochloremia in Chronic Respiratory Acidosis. Howard Levitin, William Branscome, and Franklin H. Epstein,* New Haven, Conn.

Acute studies of $\mathrm{CO}_{2}$ inhalation in man and animals have heretofore failed to demonstrate losses of chloride in the urine in excess of sodium. It has even been suggested by some that the characteristic hypochloremia of respiratory acidosis might arise from transfer of chloride to an intracellular position or from dilution of the extracellular fluid with sodium bicarbonate rather than from increased excretion of chloride by the kidneys.

Rats were placed in metabolism cages in an atmosphere containing varying concentrations of $\mathrm{CO}_{2}$. One group was allowed food and water ad lib. during 2 to 3 days of exposure to $71 / 2$ per cent $\mathrm{CO}_{2}$ or 12 per cent $\mathrm{CO}_{2}$ in air. A second group, trained to take their 24-hour requirement of food in a 30-minute feeding interval at the beginning of each day, was studied during a control period of three days in room air and then placed in 5 per cent $\mathrm{CO}_{3}$ or 10 per cent $\mathrm{CO}_{2}$ for 24 hours. All rats were exsanguinated at the end of each experiment.

Breathing $\mathrm{CO}_{2}$ produced an elevation in serum bicarbonate and a reciprncal depression of serum chloride of about $5 \mathrm{mEq}$. per 1 . with 5 per cent $\mathrm{CO}_{2}, 10 \mathrm{mEq}$. per $\mathrm{L}$. with $71 / 2$ per cent $\mathrm{CO}_{2}$, and $20 \mathrm{mEq}$. per $\mathrm{L}$. with 12 per cent $\mathrm{CO}_{2}$. These alterations were as marked at the end of one day as after three days. Renal losses of $\mathrm{Cl}^{-}$were increased on the first day of hypercapnia to produce a negative balance of $\mathrm{Cl}^{-}$at the end of 24 hours after which no further net loss occurred. Negative balances of $\mathrm{K}^{+}$and sometimes of $\mathrm{Na}^{+}$accompanied chloruresis in rats of group I but not of group II. Excretion of $\mathrm{NH}_{4}^{+}$ increased on exposure to $\mathrm{CO}_{2}$ and returned promptly to normal when the rats were given room air to breathe.

These experiments imply that renal compensation, characterized by the excretion of $\mathrm{Cl}^{-}$, plays an important role in adjustments to respiratory acidosis in the rat. They further suggest that the chloruresis induced by breathing $\mathrm{CO}_{2}$ may provide a convenient tool for the study of renal tubular mechanisms for the excretion and reabsorption of anions.

\section{An Immunological Approach to Problems of Blood Co- agulation. Jessica H. Lewis * and Paul Didisheim, Pittsburgh, $\mathrm{Pa}$.}

Following repeated injections of certain human plasma or serum fractions, rabbits developed high titer of antibodies, demonstrable by precipitin reactions. In addition the antisera developing in the injected rabbits specifically inhibited certain human coagulation factors but not the rabbits' own factors. A citrate eluate of barium sulfated normal serum was shown to contain high concentrations of the clotting factors PTC (plasma thromboplastin component) and proconvertin (Factor 7) and traces of prothrombin. After injection with this fraction rabbits developed antibody with marked anti-PTC and anti-proconvertin activity and moderate anti-prothrombin activity. 
If a similar fraction was prepared from serum of a PTC deficient individual, the fraction contained proconvertin and traces of prothrombin, and after immunization with this fraction the rabbit anti serum showed no anti-PTC, but marked anti-proconvertin and a trace of anti-prothrombin. This anti-prothrombin, and probably other nonidentified inhibitors, could be removed by adsorption with plasma from a patient congenitally deficient in proconvertin (Stuart). After absorption the anti serum still showed marked clot inhibiting effects, prolonging normal plasma recalcification time, preventing normal thromboplastin generation and reducing proconvertin titer as tested by Owren's method or a two-stage method on isolated materials. Control uninjected rabbit plasma did not show any inhibitor or precipitin reaction with normal human plasma. The injected rabbits showed normal coagulation mechanisms.

The demonstration that antibodies to human PTC, proconvertin and prothrombin can be formed suggests that, in the future, precipitin reactions employing specific antibodies may be useful as methods for assaying human clotting factors.

Stimulation of Adrenocortical Secretions after Air Encephalography. Amos H. Lieberman and JoHn A. Luetscher, JR.,* San Francisco, Calif.

Aldosterone output appears to be dependent on alterations in the volume of a component of the extracellular fluid, while 17-hydroxycorticoid excretion is not affected by similar changes in hydration. To determine the effect of acute reduction in cerebrospinal fluid volume, seven patients have been studied before and on the day of air encephalography.

In four patients, no effort was made to control fluid or food intake. On the day of encephalography, there was a regular fall in urine volume and sodium content, while no consistent change in potassium excretion was seen. Aldosterone output increased two to three fold. Initial levels of 17-hydroxycorticoids of 4.7 to $7.1 \mathrm{mg}$. rose to 8.6 to $18.6 \mathrm{mg}$. per day. There was no consistent change in 17 -ketosteroids.

In order to minimize dehydration and sodium depletion, oral intake was supplemented by intravenous fluids in three other patients, so that water, sodium, and potassium intake approximated control levels. On the day of encephalography, urine volume fell, and sodium output was unchanged. Potassium exrretion rose in two cases. Aldosterone output increased in two cases. Elevation in 17-hydroxycorticoid excretion was similar to that observed in previous cases.

Acute reduction in volume of cerebrospinal fluid is followed by increased adrenocortical secretion of aldosterone and 17-hydroxycorticoids. These changes are not entirely dependent on dehydration or electrolyte depletion. Similar increases in aldosterone and 17-hydroxycorticoids have been noted after acute stimulation with exogenous corticotropin.
Quantitative Aspects of the Stimulus Resulting in Pain in Esophagus, Small and Large Intestines. MARTIN Lipkin and Marvin H. SLEISEnger, New York, N. Y. (Introduced by Robert F. Watson).

The relationship of stimulus intensity and duration required to produce pain in esophagus, ileum and colon has been studied. In each experimental determination a column of water was suddenly connected to a water-filled balloon-catheter system previously placed in the lumen of the organ. The height of the column of water was maintained constant during each determination. The magnitude of the pressure source and the time to onset of pain were measured. The experimental apparatus was identical in all determinations. Normal subjects were chosen for measurements of esophagus and colon, and subjects with ileostomy for measurements of ileum.

For each of the three organs it was found that as the intensity of the stimulus decreased, the duration required to produce pain increased. Values comprising the intensity-duration curves plotted for the three organs were found to be very similar. The variability of responses was greatest in the colon. Pressure declines in the tubing, and elastic properties of the balloons were measured, and it was determined that the intensity-duration curves would remain qualitatively unchanged if the stimulus intensity values were corrected to the actual pressure applied to the wall of the viscus.

The results of the study suggest that the rate of onset of pain is a function of the rate of distension of the viscus. To evaluate this possibility, an experimental procedure has been developed to measure the rate of distension and pressure applied directly to the wall of the viscus during distension.

\section{Metabolic Studies of Sodium Depletion in Adrenalec-} tomized Humans. MORTIMER B. LIPSETT and OloF H. Pearson,* New York, N. Y.

The detailed adjustments to salt depletion and subsequent repletion are difficult to study in man because of the efficient renal conservation of sodium. However, the continued renal salt loss, which occurs in the adrenalectomized human receiving a low salt diet and maintained with cortisone, permits observations of the phenomena accompanying sodium depletion.

Three adrenalectomized patients and one with Addison's disease have been followed with metabolic balance techniques through periods of low and high salt intakes to induce sodium depletion and repletion. Negative sodium balances of $600 \mathrm{mEq}$. were reached in two patients with initially normal total exchangeable sodiums before symptoms developed. The two other patients with initially low total exchangeable sodiums (21 and $23 \mathrm{mEq}$. per kilogram) developed hyponatremia and became moderately ill after net sodium losses of 135 and $180 \mathrm{mEq}$.

During the early stages of salt withdrawal, the negative sodium balance and decrease in weight suggested loss of extracellular fluid. As sodium depletion continued, the weight loss did not keep pace with the salt loss, implying some conservation of water in the face of con- 
tinued sodium depletion. During this latter period, hyponatremia developed.

Although cortisone replacement remained constant, salt depletion was accompanied by negative nitrogen balances of 1 to 2 grams per day and creatinuria. This negative nitrogen balance was reversed and the creatinuria dropped sharply when salt was returned to the diet.

Positive sodium balances as high as $1,000 \mathrm{mEq}$. occurred with sodium repletion. Initially, however, there was little weight gain, although plasma volumes and chloride spaces expanded and the sodium entered the chloride space. This has been interpreted to mean that, although exogenous water was available, the first response to sodium replacement was a shift of intracellular water into the chloride space. These phenomena emphasize the complexity of the problems associated with "uncomplicated" salt loss.

Production of Carbon Monoxide by Hemin Oxidation. G. D. Ludwig, W. S. Blakemore, and D. L. Drabkin, Philadelphia, Pa. (Introduced by F. C. Wood).

The production of $\mathrm{CO}$ by oxidation of hemoglobin and hemin has been demonstrated in vitro. These studies support Sjöstrand's thesis that $\mathrm{CO}$ is formed in man and other animals in the destruction of hemoglobin. More detailed investigation of the coupled oxidation of crystalline hemin (pyridine hemochromogen) and of various reducing substances (hydrazine, ascorbic acid) shows that $\mathrm{CO}$ is produced concomitantly with green pigment (verdohemochromogen) and results from fission of the ring at the $\alpha$-methene bridge carbon. In different phases of these experiments $\mathrm{CO}$ has been measured by 1 . Infrared gas analyzer; 2 . Spectrophotometric recording of $\mathrm{CO}$ complexes of hemoglobin and of alkaline cytochrome c; Chemical methods dependent upon reaction with 3. Iodine pentoxide or 4. Palladium chloride; 5. Production of radioactive $\mathrm{BaCO}_{3}$ following oxidation of $\mathrm{CO}$ formed from labeled hemin.

Crystalline hemin, containing 8 labeled $\mathrm{C}^{14}$ atoms per molecule (each methene bridge $C$ and an adjacent $C$ in each pyrrole), was oxidized by the Warburg-Negelein method; the resultant $\mathrm{CO}$ quantitatively converted to $\mathrm{CO}_{2}$ and precipitated and counted as $\mathrm{BaCO}_{8}$. The specific activity of the recovered radioactive $\mathrm{CO}_{2}$ permitted the conclusion that the $\mathrm{CO}$ arose from one of the four methene bridge carbons (presumably the $\alpha$-methene $C$ ), since no evidence of dipyrrole formation or further degradation of pyrroles was obtained and Lemberg has demonstrated that the bile pigment produced by this reaction is of the $\alpha$-urobilin type.

Kinetic studies show that the peak of $\mathrm{CO}$ production lags far behind that of an intermediate verdohemochromogen, in which the $\alpha$-methene $C$ is oxidized to a carbonyl group, as claimed by Fischer and others.

Assuming that the same yield of $\mathrm{CO}$ per molecule of hemin decomposed is obtained in vivo as in vitro, then the amount of $\mathrm{CO}$ that is produced in the daily catabolism of hemoglobin is a major factor in the blood CO concentrations found in non-smoking healthy subjects.
A Pathophysiological Study of Myoclonus Due to a Virus. Charles N. Luttrell and Frederick B. Bang,* Baltimore, Md.

Myoclonus is a characteristic manifestation of Newcastle disease virus encephalomyelitis in chickens, mice, cats and monkeys. This study analyzes myoclonus and encephalomyelitis in experimentally infected cats.

Intracerebral, intracerebellar and intracervical cord innoculation of the virulent strain of Newcastle disease virus regularly produced encephalomyelitis in adult cats. In kittens, moreover, this was achieved by intranasal and intraocular routes. They, like adult cats, developed an encephalomyelitis with myoclonus.

Electroencephalograms and electromyograms were obtained serially in seven animals, and suggested the myoclonus originated from a deep lying subcortical focus or foci.

Serial sections of brains and cords of cats infected by the following routes: 5 intranasal, 4 intraocular, 5 intracervical cord, 2 intracerebral and 2 intracerebellar were examined and compared with two controls. The preponderant histological abnormalities were found in hypothalamus, brain stem and spinal gray. The type and distribution of lesions were similar to those of encephalitis lethargica. The histopathological similarities of these two encephalitides associated with the increasing variety of viruses belonging to the myxovirus group which cause encephalitis in both man and animals suggest the possibility that encephalitis lethargica was a member of this group.

A total of 12 brain stem and 8 high spinal transections were performed in cats with Newcastle disease virus encephalomyelitis and myoclonus. In $11 / 12$ of brain stem and $7 / 8$ of spinal animals the character and rate of the myoclonus were unchanged. In these cats it seems reasonable to attribute myoclonus to some "pathophysiological" change occurring at brain stem level or below. Moreover, it is clear that myoclonus in cats with Newcastle disease virus encephalomyelitis can occur at the organizational level of spinal cord, and that suprasegmental structures are not necessary for its development or maintenance.

The Uptake and Metabolism of $S^{\text {s }}$ Labeled Thiourea, Thiouracil, and Potassium Thiocyanate by the Thyroid and Other Tissues. F. Maloof and M. Soodak, Boston, Mass. (Introduced by J. Lerman).

Thiourea, thiouracil and potassium thiocyanate (KSCN) have been utilized to study the metabolism of iodide by the thyroid for over a decade, yet the precise mode of action of these drugs remains unknown. Observations were made following the administration of these compounds, labeled with radioactive sulfur $\left(S^{*}\right)$, to rats and following the incubation of these compounds with thyroid slices and thyroid homogenates.

These compounds reach their site of action in the thyroid within minutes following their administration to rats. Only a small fraction ( 0.5 to 2.0 per cent) of the administered dose is taken up by the thyroid. There is 
no concentration gradient for these compounds in the thyroid versus serum. Furthermore, the observations reveal that their uptake by the thyroid is not under the influence of the pituitary and is not depressed by compounds which lower the uptake of $\mathrm{I}^{100}$ by the thyroid.

The experiments with rats demonstrate an interesting and unique degradation of these $S^{35}$-labeled compounds by the thyroid in contrast to the liver and serum. There appear to be several common denominators involved in the metabolism of $\mathrm{S}^{3}$-thiourea and the iodination mechanism in the rat thyroid: (1) both are greatly diminished by hypophysectomy; (2) both are inhibited by the administration of Sulfadiazine, and (3) the amount of $S^{{ }^{*}}$ thiourea metabolized and the amount of thyroxine formed per day by the rat thyroid are of the same order of magnitude.

$\mathrm{KSCN}$ is known to displace iodide from the thyroid. The goitrogenic effect of $\mathrm{KSCN}$ can be overcome by iodides. The present data demonstrate that iodide does not displace $\mathrm{KSCN}$ from the thyroid, but rather inhibits its degradation in the thyroid.

These studies establish the minimal concentration of these compounds in the thyroid necessary to inhibit the concentration of $\mathrm{I}^{180}$ and the formation of protein bound $\mathrm{I}^{180}$ by the thyroid, approximately $2.6 \times 10^{-5} \mathrm{M}$.

\section{A Relationship Between Human Erythrocyte Aging In $V$ ivo and the Activities of Glucose-6-Phosphate and 6-Phosphogluconic Dehydrogenases. PAUL A. Marks, New York, N. Y. (Introduced by Alfred Gellhorn).}

Erythrocytes derive most of their energy from glucose utilization. It is well established that the pentose phosphate pathway ( $P P P)$, in addition to anaerobic glycolysis, plays an important role in RBC metabolism. Little is known, however, about the changes occurring in carbohydrate enzyme levels associated with red blood cell ( $R B C$ ) aging in vivo.

PPP activity in RBC was evaluated by applying to hemolysates specific assays for glucose-6-phosphate dehydrogenase (G-6-P.D.) and 6-phosphogluconic dehydrogenase (6-P.G.D.), which respectively catalyze the reactions : glucose-6-phosphate + triphosphopyridine nucleotide $(T P N) \rightleftharpoons 6$-phosphogluconolactone + reduced TPN (TPNH) and 6-phosphogluconate $+\mathrm{TPN} \rightleftharpoons$ ribulose-5phosphate $+\mathrm{CO}_{2}+\mathrm{TPNH}$.

Average dehydrogenase activities, expressed in units $/ 10^{\circ}$ $\mathrm{RBC}$, in 30 normal subjects were: G.-6-P.D. $443 \pm 108$ and 6-P.G.D. $660 \pm 128$. Patients with reticulocytosis associated with various types of hemolytic anemia (6), hemorrhage (2), neoplastic disease (3), and therapy of iron deficiency anemia (3) and pernicious anemia (1), had significant elevations in activities of G.-6-P.D. (range 680 to 1160 ) and 6-P.G.D. (range 920 to 1420). In 13 diabetics not requiring insulin and 19 neoplastic disease subjects, the average enzyme activities did not differ significantly from normal.

Reticulocytes and young mature RBC compared to older $\mathrm{RBC}$ are less dense and more resistant to hypotonic hemolysis. Following centrifugation of both normal bloods (reticulocytes $<1.0$ per cent) and hemolytic anemia blood (reticulocytes: 35 per cent) the upper RBC layer had higher enzyme activities than the lower RBC layer. Subjecting aliquots of normal bloods to graded hypotonicity revealed lower enzyme concentrations in the less osmotically resistant $\mathrm{RBC}$.

These data strongly suggest that associated with maturation and aging of the $\mathrm{RBC}$ in vivo, there is an alteration in at least two enzymes involved in glucose metabolism. Increased levels of these enzymes may be a more sensitive indicator of young cell populations than reticulocyte counts. These enzymes catalyze reaction providing mechanisms for glucose oxidation with TPNH generation and pentose phosphate formation. A decrement in their activity may be an important aspect of $\mathrm{RBC}$ aging.

The Metabolic Effects of Glutamic Acid and Arginine in "Hepatic Coma" with Hyperammoniemia. William V. McDermotr, Jr., Dorothy H. Henneman, and Claude Laumont, Boston, Mass. (Introduced by R. D. Adams).

Recent conflicting reports on the value of L-glutamic acid in the treatment of "hepatic coma" have not clarified the biochemical effects of this amino-acid.

Ten patients with elevated levels of blood ammonia and with disturbances of the central nervous system were selected and the effects of parenteral sodium glutamate studied. Ammonia, glutamine, alpha keto-glutarate, phosphate, citrate, pyruvate and lactate were measured before, immediately after the infusion and at various times in the subsequent 48 hours. In all cases the administration of the glutamate infusion was attended by a precipitous fall in the blood ammonia level, by a rise in glutamine, and by lesser rise in alpha keto-glutarate. Any other biochemical changes observed were neither consistent nor of sufficient magnitude to permit interpretation. Subsequent to the infusion, the ammonia level rose slowly and concomitantly the glutamine and alpha keto-glutarate levels fell. These observations suggest that the administration of L-glutamic acid to patients with hyperammoniemia is attended by a temporary binding of the ammonia in the form of glutamine and a further reaction of glutamine to form alpha keto-glutarate. The fact that this reaction is reversible may explain the conflicting clinical reports on the value of this amino-acid.

The effects of infusions of arginine hydrochloride were quite different. No immediate changes occurred but within 12 to 24 hours a steady fall in blood ammonia levels was observed. Glutamine and alpha keto-glutarate were unchanged. Corresponding improvement in clinical and electroencephalographic abnormalities occurred. These changes were long-lasting and have persisted for several weeks without further treatment.

It would appear that both amino-acids could be used to advantage, in the treatment of ammonia intoxication, glutamic acid for its immediate binding effect on circulating ammonia and arginine for its slower but more permanent effect on the clearance of abnormal amounts of ammonia. 
The Production and Catabolism of Cortisol in Experimental Endotoxin Shock. James C. MELBY, Richard A. DeWall, James L. Storey, and Richard H. EgDAHL, Minneapolis, Minn. (Introduced by Wesley W. Spink).

The shock induced in dogs by endotoxin from Gramnegative bacteria is characterized by elevated 17-hydroxycorticosteroid concentrations in the plasma and the gradual disappearance of cortisol from the plasma when given intravenously. Adrenal cortical secretory activity was assessed directly by the measurement of the minute output of 17-hydroxycorticosteroids in adrenal venous blood in dogs with adrenal vein cannulas. Prior to the injection of endotoxin the adrenal 17-hydroxycorticosteroid output rose from 0 to 1 microgram per minute to 10.7 micrograms per minute in response to corticotropin.

Following shock induced by endotoxin the output rose within two hours to an average maximum of 9 micrograms per minute and diminished thereafter. The adrenal response to endotoxin shock was abolished by hypophysectomy. Response to corticotropin had declined to an average maximum of 6.5 micrograms per minute four hours after endotoxin. There was a similar diminution of adrenal responsiveness to corticotropin following endotoxin in the hypophysectomized animal. Dogs given a constant infusion of corticotropin ( 0.2 unit per minute) averaged an output of 15.3 micrograms per minute of 17-hydroxycorticosteroids after three hours. Subsequent to the production of shock the adrenal output of 17hydroxycorticosteroids fell progressively to an average of 4.4 micrograms per minute in the presence of high concentrations of corticotropin.

These findings indicate that the elevated plasma 17hydroxycorticosteroid concentration in shock produced by endotoxin reflects both a diminished rate of steroid catabolism and markedly increased adrenal cortical secretory activity which progressively diminishes even in the presence of high concentrations of corticotropin.

The Effects of Heat and Pasteurization on Albumin Preparations in Particular Reference to Radioisotope Labeled Materials. William R. Merchant, S. P. MAsouredis, and Eric Ellenbogen, Pittsburgh, $\mathrm{Pa}$ (Introduced by T. S. Danowski).

Since its primary development as a blood volume expander, albumin in its commercial form has been used for numerous physiological and isotopic studies. Little attention has been directed to the fact that for precautionary measures this material has been pasteurized at $60^{\circ} \mathrm{C}$ for ten hours in the presence of stabilizers. In most instances only an increase in turbidity or an electrophoretic homogeneous peak in dilute solution has been used as a criterion of homogeneity of the protein. Broad inferences have been drawn from the use of this material as to the biological behavior of "native albumin."

Marked changes were observed even electrophoretically in heating human or rabbit serum at $56^{\circ} \mathrm{C}$ or $60^{\circ} \mathrm{C}$ for varying periods of time, where the alteration was reflected in increased peaks in the positions of alpha 2 and beta globulins and in the albumin peak splitting into two components.

Preliminary light scattering studies on commercial albumin preparations would indicate the weight average molecular weight of the solute is in the neighborhood of 300,000 , compared to a light scattering molecular weight of 75,000 for pure crystalline human serum albumin.

It was still possible that biologically this heated material would behave as the unheated. Fraction V of rabbit plasma was prepared by Cohn's method six, one part treated as a commercial preparation, the other simply diluted with physiological saline. Both were labeled with $\mathrm{I}^{\mathrm{ra1}}$ and injected into rabbits. Marked differences in the biological half life were observed, with the heated material disappearing more rapidly from the plasma.

The above would suggest that insufficient attention has been directed to establishing biological and biophysical criteria for proteins administered for clinical studies.

\section{Active Function of Renal Tubules During Acute Tubular} Necrosis. W. H. Meroney and M. E. Rubini, Washington, D. C. (Introduced by Stanley M. Levenson).

The opinion is generally stated that during the oliguric phase of acute tubular necrosis the glomerular filtrate re-enters the extracellular fluid by passive back diffusion through discontinuous or inert tubular walls, and the small portion which escapes to the bladder resembles an ultrafiltrate of plasma. The present observations are inconsistent with this view and indicate that most of the urine derives from a few essentially normal tubules functioning under intense stimulation.

In ten patients with oliguria following hypotension, the osmolar U/P approximated unity. Urinary sodium concentration, always lower than plasma, fell progressively on successive days of oliguria. Urinary potassium concentration, always higher than plasma, increased as sodium decreased. The downward trend of urinary $\mathrm{Na} / \mathrm{K}$ ratio was abruptly reversed by hemodialysis, but the ratio did not return to the high values present previously, when the plasma abnormalities were similar, until diuresis occurred.

Glycosuria did not appear, although some patients had induced hyperglycemia. In other oliguric patients extreme, sustained hyperglycemia was produced without the appearance of glycosuria. During diuresis hyperglycemia caused glycosuria.

Sodium conservation and potassium secretion, which were shown by dialysis to be partially independent of plasma concentrations, improved before urinary volume increased, suggesting an adaptive response of tubules already functioning rather than the contribution of newly healed nephrons. Inactive tubules could not have contributed significantly to the final urine without reducing the urine:plasma difference and increasing the urinary volume. The absence of glycosuria during hyperglycemia further demonstrates tubular activity and implies a severe reduction in glomerular filtration rate.

During diuresis the trickle of processed urine from the few active tubules is obscured by an overwhelming flood 
of "ultrafiltrate" conducted by the most severely damaged tubules, which are newly patent but still unhealed.

\section{Abnormalities of Blood Coagulation Produced in Rabbits by Feeding Cholesterol. Clarence Merskey, Cape- town, South Africa. (Introduced by David Gitlin).}

Twenty-seven adult male rabbits were fed an adequate diet for 96 days. Cholesterol (600 or $1,000 \mathrm{mg}$. daily) was fed to 18 of these, which produced coagulation defects as judged by prolongation of coagulation time (in 12) and prothrombin consumption defects (in 15). Plasma prothrombin and factor VII were markedly increased. Blood thromboplastin generation was defective, the abnormality lying in the serum factor, the alumina treated plasma being exceptionally active. Addition of normal or hemophilic serum corrected this serum defect, but serum from patients on phenindione therapy only partially corrected it whereas Christmas disease serum had no corrective effect. This suggested that the affected serum lacked Christmas factor, but this was not confirmed by direct mixing experiments. Affected rabbit blood added to Christmas disease or hemophilic blood shortened the coagulation time and corrected the prothrombin consumption defect of both these bloods if added in small proportion, but not in either if added in larger proportion. Blood or affected rabbits prolonged coagulation time and caused prothrombin consumption defects if mixed with normal blood. These findings suggested the presence of a circulating anticoagulant and possibly a defect of a third serum component (factor $\mathrm{X}$ ?). It is possible that the mere presence of high concentrations of cholesterol (up to 2,000 mg. per cent) in the blood may cause the effects described, and preliminary studies in human hypercholesterolemic states lend some support to this concept.

\section{Abnormal Binding of Vitamin $B_{12}$ by Sera from Patients with Chronic Myelogenous Leukemia. A. MILLER, Boston, Mass. (Introduced by Charles P. Emerson).}

Increased serum concentrations of vitamin $B_{1}$ and prolonged disappearance times of intravenous cobalt ${ }^{\infty 0}$ labelled $B_{12}$ have been noted in chronic myelogenous leukemia. In the present study, sera from normal subjects and patients with blood dyscrasias have been tested for their vitamin $B_{13}$ binding capacities by an equilibrium dialysis method and analyzed for their vitamin $B_{1}$ concentrations microbiologically.

In 22 normal subjects, serum $B_{19}$ concentrations ranged from 270 to 1,250 micromicrograms per milliliter and binding capacities from 630 to 2,185 micromicrograms per milliliter. In 11 patients with chronic myelogenous leukemia, serum $B_{1 s}$ concentrations ranged from 4,200 to 14,000 micromicrograms per milliliter and binding capacities from 4,750 to 14,000 micromicrograms per milliliter. With treatment, binding capacities declined rapidly, reaching normal levels in two to three weeks, whereas serum $B_{1}$ concentrations remained unchanged for several weeks and then slowly declined. Increased serum $B_{12}$ concentrations and binding capacities were not observed in acute leukemia, chronic lymphocytic leukemia, polycythemia vera, and five of six patients with leukemoid reactions. One patient with chronic monocytic leukemia had levels similar to those in chronic myelogenous leukemia.

Sera to which excess $\mathrm{C}^{\infty} \mathrm{B}_{1 \mathrm{~s}}$ had been added were dialyzed to completion and then fractionated by paper electrophoresis. The $\beta$ globulin contained the major fraction of the bound $\mathrm{Co}^{\infty} \mathrm{B}_{13}$ in 6 of 7 normal sera whereas the $\alpha_{1}$ globulin was the chief binding protein in 5 of 7 chronic myelogenous leukemic sera. The $\alpha_{1}$ globulin contained 24 to 56 per cent of the bound $B_{13}$ in patients with chronic myelogenous leukemia as compared to 3 to 9 per cent in normal subjects.

The increased serum $B_{1}$ concentration and binding capacity in chronic myelogenous leukemia, associated with an increased binding of the labelled vitamin by the $\alpha_{1}$ globulin, suggest a specific abnormality of serum proteins in this disease which is not generally found in leukemoid states and other blood dyscrasias.

\section{A Study of Etiology of Macrocytic Anemia. O. Neal} Miller and Walter G. Unglaub, New Orleans, La. (Introduced by Grace A. Goldsmith).

Forty-six patients with macrocytic anemia have been studied with three procedures: 1) Schilling Test, 2) estimation of intrinsic factor (IF) in gastric juice and 3) estimation of serum factor. Methods for the last two procedures have been developed in this laboratory based on the previous observation that materials which contain intrinsic factor potentiate the ability of serum to combine with vitamin $B_{12}$. The patients were divided into groups according to their response to the Schilling Test. When absorption of radioactive $B_{18}$ was stimulated by including IF concentrate with the radioactive test dose, the patient was classified as pernicious anemia (PA). Patients who showed normal absorption either with or without added IF were designated as nutritional macrocytic anemias (NMA). A third group absorbed little vitamin $B_{1 s}$ with or without added IF and was classified as malabsorption syndrome (MAB). It was found that gastric juice from PA patients had only 25 to 30 per cent as much IF activity per $\mathrm{ml}$. as normal gastric juice and similarly NMA and MAB patients had 70 per cent and 15 to 20 per cent, respectively. Serum factor for these three groups, as compared to pooled normal serum, averaged, 70 per cent for PA, 80 per cent for MAB and 100 per cent for NMA. Several patients having been diagnosed and treated for many years as PA were found to be MAB by these three procedures. Patients with MAB were lower in IF than patients with PA; both were low in serum factor. In NMA either serum factor or IF was low but not both. These data suggest that both intrinsic factor and serum factor are necessary to prevent all three types of macrocytic anemia.

The Relation of Maximal Oxygen Uptake to Cardiac Capacity and Arterial Oxygen Saturation in Normal Subjects and in Patients with Mitral Stenosis. JERE Mitchell and Carleton B. Chapman,* Dallas, Tex.

Maximal oxygen uptake is thought to provide an index to cardiac pumping capacity in the absence of sig- 
nificant pulmonary disease. Prior to testing this thesis, maximal oxygen uptake was determined in 73 normal men, aged 20 to 65 , using a motor-driven treadmill. The value was found to decline with age. In 10 normal subjects, oxygen uptake and cardiac output (dye dilution method) were measured at increasing work loads. The mean maximal oxygen uptake was $3443 \pm 430 \mathrm{cc}$. At this level, mean cardiac output had increased from $6.5 \pm 1.7 \mathrm{~L}$. per min. (resting, standing) to $26.1 \pm 6.5 \mathrm{~L}$. per min. At work loads greater than that producing maximal oxygen uptake, cardiac output tended to decline.

Determination of arterial oxygen content, $\mathrm{PaO}_{2}$ (polarographic), oxygen capacity, and hematocrit during work showed no evidence of arterial desaturation or decline in $\mathrm{PaO}_{2}$, even at loads producing maximal oxygen uptake. Simultaneous ear oximetry, however, regularly showed decline in saturation readings, apparently owing to factors unrelated to oxygenation of arterial blood.

In 7 patients with clinically significant mitral stenosis, maximal oxygen uptake was about half that found in normal subjects of comparable age. Simultaneous cardiac output measurements in 2 of the 7 showed very slight increases as work loads were raised. T-1824 appearance times tended to become longer with increase in work; in normal subjects, on the other hand, appearance time progressively shortened under the same conditions.

The studies support the thesis that maximal oxygen uptake is determined primarily by cardiac capacity in subjects without significant pulmonary disease. They also show that exercise of this type does not ordinarily produce arterial desaturation. Mitral stenosis lowers cardiac capacity and maximal oxygen uptake; the contours of T-1824 dye curves recorded during exercise in such patients come to resemble those seen in patients with cardiac failure as exercise loads are increased.

The Effect of Insulin, Alloxan Diabetes, and Phlorhidzin on Sugar Transport Across the Muscle Cell Membrane. H. E. Morgan and C. R. PARK,* Nashville, Tenn.

The perfused isolated rat heart is a desirable test object for studying the transport of sugars through the muscle cell membrane. The preparation has no broken cells; sugars reach all cells rapidly due to the intact circulation and contraction. Viability is maintained for 1 hour or more without exogenous substrates.

Transport into the cell of the non-metabolized pentose, L-arabinose, can be determined by its intracellular accumulation. Intracellular sugar is estimated as the total tissue sugar minus the extracellular sugar. The extracellular space is determined simultaneously with the nontransportable sorbitol-1-C $C^{14}$. Inward glucose transport is measured by disappearance from the perfusate.

Transport out of the cell is measured by perfusion of a heart containing intracellular L-arabinose with pentose free medium. After the initial 3 minutes, which removes 99 per cent of extracellular sugar, the arabinose appearing in the medium comes from inside the cells.

Glucose in the medium markedly inhibits the accumulation of intracellular L-arabinose; conversely, in high concentration L-arabinose inhibits glucose disappearance from the perfusate. Inward transport of each sugar is strongly inhibited by $3 \times 10^{-3} \mathrm{M}$ phlorhidzin and by alloxan diabetes. Transport is strongly accelerated by insulin.

The outward transport of L-arabinose is decreased by 48 hours fasting and alloxan diabetes. Insulin ( $3 \mu \mathrm{g}$. per ml.) causes a striking increase in outward transport approaching a maximum in about 5 minutes. The hormone effect is slower in the diabetic heart.

Both sugars share a reversible transport system distinct from diffusion and not involving phosphorylation. Transport is a site of the opposing hormonal actions of insulin and certain diabetogenic factors which thus regulate glucose supply in muscle. The metabolic action of phlorhidzin can be explained by transport inhibition.

Abnormal Cholesterol Metabolism in Lymphoma: A Comparison of Findings in Serum and Splenic Tissue. Elly Moschides and Mario Stefanini,* Boston, Mass.

Abnormalities of cholesterol metabolism have been described previously in serum of patients with lymphoma (Am. J. M. Sc., 1956, 231, 155). They include: 1) low total cholesterol and cholesterol esters; 2) decrease of cholesterol migrating with the beta globulins fraction; 3) increase of cholesterol migrating with the albumin and alpha-1 globulins fractions. To elucidate the mechanism of these changes we have studied: a) content of total cholesterol and cholesterol esters; b) cholesterol esterases activity; c) distribution of cholesterol among the various protein electrophoretic fractions in the splenic tissue of 7 patients with lymphoma. These values have been compared to those obtained with serum. Splenic tissue was obtained surgically and immediately frozen in liquid $\mathrm{N}_{2}$ after washing with cold saline. Autopsy or surgical spleens from patients with miscellaneous disorders represented a control group.

Findings in lymphoma: 1) Significant elevation of total cholesterol in splenic tissue as compared to a significant drop in serum. This was, however, a not specific finding which was noted in all pathologic spleens, irrespective of the disease entity involved. 2) Striking defect of esterification of cholesterol in both splenic tissue and serum of patients with lymphoma (and leukemias). Preliminary studies have indicated that such finding may be explained by decrease of cholesterol esterase activity in splenic tissue. 3) Relative increase of cholesterol-carrying beta globulins in splenic tissue. 4) Decreased cholesterol binding capacity of beta globulins in serum. It would then appear that defective enzymatic mechanisms and alterations in the tissues/serum balance of cholesterolbinding beta globulins might induce the striking alterations of cholesterol metabolism observed in patients with lymphoma.

These studies are among the first to indicate a definite metabolic abnormality in lymphoma; they stress the potential importance of biochemical studies of tissues in the interpretation of the pathogenesis of disease states. 
Some Observations of the Effect on Serum Cholesterol of an Unsaturated Oil Administered Intravenously to Normal Healthy Adult Men. JоH N. MUELler,* JACK M. Iacono, and Alian L. Forbes, Denver, Colo.

The serum cholesterol-lowering potency of fed unsaturated fatty acids is not clearly understood. Intravenous fat emulsions provide an excellent tool to study this phenomenon, since the important effects of absorption can be by-passed.

Total, esterified and free cholesterols were measured in whole serum and its subnatant (spun at 12,000 rpm for 45 minutes) in 10 healthy young adult males of comparable ages before and at intervals after the intravenous injection of $90 \mathrm{gms}$. of a highly unsaturated oil. Six men received one injection separated by one week from oral ingestion of the same fat load. The other four received 5 daily infusions, cholesterol analyses being performed on the first, third, and fifth days.

In both groups there is a transient rise in whole serum cholesterol following the infusion which reaches its peak in four hours and then begins to fall. By 24 hours it is 18 per cent below the pre-infusion level. With oral administration there is no immediate rise and the fall is not so great, amounting to 11 per cent after 24 hours. In the four subjects receiving multiple infusions, the fall was progressive so that after 5 days the serum cholesterol was down 27 per cent.

The pattern for the subnatant or high density lipoprotein cholesterol was strikingly different. It fell precipitously during the infusion and continued to decline during the first 24 hours. Thereafter, each day the fall was less, so that on the fifth day the subnatant cholesterol actually rose. As with whole serum, the changes occurred primarily in the esterified fraction.

The rise in the whole serum cholesterol occurring simultaneously with a fall in subnatant at a time when the infused fat is rapidly disappearing from the blood suggests that endogenous cholesterol is utilized as a fat carrier or emulsifier, becomes associated with the low density or chylomicron lipoprotein component and disappears from the blood in this manner. Thus an overall decrease in serum cholesterol is attained.

The Hemodynamics of Mitral Valvular Disease. PAUL Novack, Robert C. Schlant, Florence W. Haynes, and Arthur O. Phinney, Jr., Boston, Mass. (Introduced by Lewis Dexter).

Left heart catheterization was employed in the study of 41 patients with severe mitral disease. Aortic flow (AO) was estimated by the indicator dilution method, regurgitant flow (RF) by the Korner-Shillingford method, and mitral valve area (MVA) by the Gorlin formula. Patients were divided into three groups based on MVA : Group I, 15 patients, MVA $<1.1$ sq. cm. (predominant mitral stenosis); Group II, 11 patients, MVA 1.1 to $1.4 \mathrm{sq}$. $\mathrm{cm}$. (mitral stenosis and insufficiency); Group III, 15 patients, MVA $>1.5$ sq. cm. (predominant mitral insufficiency). AO was greatly reduced in Groups I and II, averaging 2.1 (1.4 to 3.3 ) and 2.3 (1.2 to 3.1 )
L. per min. per M. ${ }^{2}$ and slightly reduced in Group III, averaging 3.1 (1.8 to 5.6) L. per min. per M.'. The means and ranges of RF for each of the groups were: 0.91 ( 0 to 1.98$), 1.35$ ( 0.47 to 2.45$)$, and 4.16 (1.50 to 7.89$) \mathrm{L}$. per min. per M.', respectively. Total ventricular output (TF) was reduced in I, normal in II, and elevated in III : 2.8 (1.4 to 3.9 ), 3.6 (2.2 to 4.9 ), and 7.3 (3.4 to 13.5) L. per min. per M. ${ }^{2}$, respectively. Significant mitral diastolic gradients occurred in all groups, averaging 23 (11 to 37 ), 14 ( 8 to 25 ), and 17 (9 to 27 ) $\mathrm{mm}$. Hg. Left atrial mean pressures did not differ significantly among the three groups, averaging 26 (14 to 20 ), 23 ( 7 to 38 ), and 24 (13 to 35$) \mathrm{mm}$. $\mathrm{Hg}$. Marked elevations in left ventricular end-diastolic pressures were rarely encountered even where there was X-ray evidence of greatly enlarged left ventricles. These averaged $6(-7$ to 11$)$, $8(0$ to 28$)$, and $10(1$ to 15$) \mathrm{mm}$. Hg. Central volumes, from left atrial injections, revealed a stepwise increase in the three groups, averaging 490 (230 to 804), 690 (410 to 1,100$)$ and 970 (510 to 1,490$)$ cc. These results suggest that pulmonary congestion in mitral insufficiency usually results from a high diastolic flow through a mildly stenotic valve rather than left ventricular failure.

\section{Effect of Low Protein Diets Upon Serum Cholesterol in Man. Robert E. Olson,* John W. Vester, Deha Gursey, and Doris Longman, Pittsburgh, Pa.}

Kempner (Am. J. Med. 1948, 4, 545) observed a fall in serum cholesterol of hypertensive patients given the rice diet, and this has been confirmed by others. Although the hypocholesterolemic effect of the rice diet has been attributed to its low fat content, the relative contributions of other nutrients to this effect have not been previously evaluated. Since a marked hypocholesterolemic effect of alipotropic diets deficient in methionine and choline but high in fat has been noted in rats in our laboratory, it was decided to explore the effects of low protein moderate fat diets upon serum cholesterol in man.

Seven human subjects, five of whom were hypercholesterolemic, were fed a control diet containing 100 grams of protein, 80 to 100 grams of fat (mainly of animal origin), and 300 to 350 grams of carbohydrate for periods of 1 to 2 weeks. During the last three days of the fore period, the composition of the diet was rigidly controlled ( $P$ 100, F 80, C 320). The subjects were then fed a diet containing 25 grams of protein (mainly of vegetable origin), 80 grams of fat (50 from butterfat), and 395 grams of carbohydrate for one week and then returned to the control diet for four additional days. Fasting serum cholesterol was determined every other day. All subjects showed a decrease in serum cholesterol during the period of low-protein feeding, the range being -22 to $-110 \mathrm{mg}$. per cent with an average of $-52 \mathrm{mg}$. per cent despite sizeable intake of saturated fat, and all values returned toward normal upon resumption of the control diet. Changes in body weight were minimal during these relatively short term studies.

The results suggest that dietary protein may be one determinant of serum lipid concentrations in man and 
are consistent with the epidemiological observation that populations ingesting relatively low amounts of protein have relative freedom from atherosclerosis.

The Effect of Potassium on Urinary Dilution. JACK Orloff,* Douglas Davidson, and Henry N. Wagner, Jr., Bethesda, Md.

The initial step in the elaboration of hypotonic urine is thought to be reabsorption of sodium and attendant anion from isosmotic precursor urine in an area of limited permeability to water. Part and possibly all transport of sodium out of tubular fluid is effected by exchange for hydrogen and potassium ions. Secretion of hydrogen ions in exchange for sodium with a secondary mechanism for anion removal will decrease the concentration of osmotically active substances in urine, forming solute-free water $\left(\mathrm{C}_{\mathrm{B}_{2} \mathrm{O}}\right)$. On the other hand, secretion of potassium in exchange for sodium will not alter urine osmolality. Furthermore, the inhibitory effect of potassium on hydrogen secretion, and consequent decrease in sodium reabsorption, were this to occur at the diluting site, should interfere with the production of $\mathrm{C}_{\mathrm{H}_{2} \mathrm{O}}$. To test this hypothesis the effect of intravenous potassium chloride on the excretion of $\mathrm{C}_{\mathrm{H}_{2} \mathrm{O}}$ in dogs was examined. Hypotonic saline was infused at a rate of approximately 1,000 micromoles per minute. When urine was maximally dilute, indicative of anti-diuretic hormone (ADH) suppression, potassium chloride was gradually substituted in equiosmolar amounts for sodium chloride. $\mathrm{C}_{\mathrm{H}_{20} \mathrm{O}} \mathrm{di}-$ minished in all studies in association with a rise in plasma potassium and an increase in potassium excretion. The fall in $\mathrm{C}_{\mathrm{H}_{2} \mathrm{O}}$ occurred despite an increase in inulin clearance, sodium excretion and total solute excretion. In contrast, enhanced potassium excretion produced by Diamox ${ }^{\circledR}$ did not effect a fall in $\mathrm{C}_{\mathrm{H}_{3} \mathrm{O}}$. This may indicate a different site of action of this drug.

Although it is not possible at this time to exclude a stimulatory effect of the elevated plasma potassium on $\mathrm{ADH}$ secretion, the results are consistent with a direct renal effect of potassium. The enhanced secretion of potassium and the interference with hydrogen ion secretion may limit the extent of urinary dilution.

Personality, Behavior, and Vasopressor Mechanisms in Hypertension. AdRIAN M. OstFeld and BenJaMin F. Lebovirs, Chicago, Ill. (Introduced by Mark $\mathrm{H}$. Lepper).

This investigation was undertaken to ascertain any predictable features of personality, behavior, or vascular reactivity in essential hypertensive subjects. During periods of tranquility and of agitation, the least concentration of levarterenol in an isotonic phosphate buffer topically applied which caused constriction of bulbar conjunctival capillaries when observed by slit lamp examination was determined and called the capillary threshold. Brachial artery pressure responses to intravenous infusion of 20 gamma per minute levarterenol, injection of five units angiotonin and $400 \mathrm{mgm}$. TEAC were determined. Periods of agitation were associated with a statistically significant rise in supine systolic and diastolic pressures, fall in capillary threshold, and decrease in fall in pressure (and in seven instances a rise) during TEAC blockade. Such altered responses during agitation occurred in nine essential, one post-toxemia, and two renal hypertensives and four normotensives.

Twenty-five stress interviews were associated with significant diastolic pressure rises in seven of eleven essential, four of five renal, and one of two post-toxemic hypertensives, one subject with Cushing's syndrome, and three of six normotensive subjects.

The psychological Test Battery consisted of WechslerBellevue Intelligence Test, Minnesota Multiphasic Personality Inventory, Bender Gestalt, Thematic Apperception Test, Rorschach, and a projective questionnaire. Twelve hypertensive demonstrated no predictable test score pattern.

The distribution of psychiatric, psychophysiological, and psychosomatic illnesses was determined in 20 hypertensives and in normotensives, similar in age, sex, race, socia class, occupation, education, and post and current residence. No difference in qualitative or quantitative distribution of these illness types was discernible in the two groups.

In this study, no personality, behavior, psychological test performance, nor vascular response has been found that would distinguish a hypertensive subject.

The Effect of Morphine Upon the Renal Excretion of Water and Solute in Man. Solomon PAPPER, LAwrence Saxon, Maurice B. Burg, Harold W. Seifer, and JaCk D. Rosenbaum,* Boston, Mass.

It is commonly stated that morphine administration stimulates the release of antidiuretic hormone with resultant decrease in the rate of urine flow. Evidence on these points and information concerning the effect of therapeutic doses of morphine on glomerular filtration rate and solute excretion in normal man are limited.

An oral water load of $20 \mathrm{ml}$. per $\mathrm{Kg}$. was established and maintained 20 times in 15 healthy subjects. During "maximal" diuresis, 7.5 to $30 \mathrm{mg}$. of morphine sulphate was administered subcutaneously, or 5 to $10 \mathrm{mg}$. intravenously. In 4 experiments the response was equivocal. In the remaining 16 studies, within less than 30 minutes urine flow decreased by 2.1 to $6.6 \mathrm{ml}$. per minute from control values of 12.1 to $22.2 \mathrm{ml}$. per minute, unaccompanied by a significant rise in urine osmolality. In 13 of the 16, the decreased diuresis was associated with a fall in endogenous creatinine clearance of 10 to $41 \mathrm{ml}$. per minute. In 14 there was a decrease in total solute excretion of 83 to 476 micro-osmols per minute.

In 7 of 20 experiments (in 5 of which nausea ensued) there was a later decrease in urine flow associated with a significant rise in urine osmolality 40 to 110 minutes after morphine administration, consistent with increased antidiuretic hormone activity.

These observations indicate that morphine, when administered in usual therapeutic doses to healthy men, may decrease the rate of water diuresis without evident re- 
lease of antidiuretic hormone. Morphine does, however, induce a prompt decrease in solute excretion and a reduction in glomerular filtration rate, changes which may account for the diminution in diuresis.

Circulation and Respiration in the Giraffe. J. L. PATterson, Jr.,* J. V. Warren,* J. T. Doyle, O. Gauer, T. KeEN, and R. H. Goetz, Richmond, Va., Durham, N. C., Albany, N. Y., Bad Nauheim, Germany, and Capetown, South Africa.

The gravitational problems in the circulation of the giraffe, and the hardly less interesting respiratory problems created by the great tracheal length, have long intrigued physiologists. The present data were obtained on four animals in the Union of South Africa.

All of the animals were "hypertensive" by human standards, with arterial pressures at heart level of 282/158 to $344 / 194 \mathrm{~mm}$. $\mathrm{Hg}$, and $360 / 257$ after bucking. Mean right atrial pressure approximated atmospheric. Pulmonary arterial pressure measured with the miniature manometer was $75 / 25 \mathrm{~mm}$. $\mathrm{Hg}$ in one animal, and enddiastolic pressure in the left ventricle 5 to $7 \mathrm{~mm}$. $\mathrm{Hg}$. Cardiac output values (T-1824 or Fick) were 49, 41 and 19.4 L. per min. When one animal's head was lowered to ground level, arterial pressure at heart level showed little change.

Tracheal volumes in two autopsied animals were 1.2 and 2.3 liters. A pressure-volume diagram on the excised lungs showed the surprisingly small total lung volume of approximately 10 liters. Respiratory minute volumes ranged from $\mathbf{7 5}$ to $\mathbf{1 7 5}$ liters per min., tidal volumes from 2.7 to 4.1 liters and $\mathrm{O}_{2}$ consumption from 2.7 to $4.7 \mathrm{~L}$. per min. Respiratory rates were 36 to 58 per $\mathrm{min}$. The inspiratory pneumotachogram in two animals was almost rectangular in shape. Both arterial $\mathrm{O}_{2}$ and $\mathrm{CO}_{2}$ tensions were normal or low normal by human standards, suggesting that in animals which were not hyperventilating the $\mathrm{PO}_{2}$ values would be low. During 10 per cent $\mathrm{O}_{2}$ inhalation, minute ventilation in the only anesthetized animal rose from 77 to 169 liters within 45 seconds.

These findings indicate that the giraffe solves the problem of cerebral perfusion by marked arterial "hypertension," doubtless aided by subatmospheric venous pressure at brain level. He probably compensates for large respiratory dead space either by large tidal and minute volumes or by adapting to some reduction in arterial $\mathrm{PO}_{2}$. His airflow pattern is unusual, and tolerance to induced hypoxia poor.

\section{Physiological Activities as a Function of Chemical Struc-} ture of Anabolic and Anti-anabolic Steroids in Man. Maurice M. Pechet, Boston, Mass. (Introduced by Mark Altschule).

The physiological effects associated with alteration of chemical structure of steroids were investigated by complete metabolic balance studies on one normal, one panhypopituitary and three Addisonian subjects with the following 16 steroids: (1) prednisone (Meti-E); (2) prednisolone (Meti-F) ; (3) cortisone (E); (4) hydro- cortisone (F) ; (5) 20ß-dihydro-prednisolone (Meti-U); (6) 17-desoxyprednisolone (Meti-A); (7) 6-dehydroprednisone (Triene-E); (8) 4-chloro-prednisone (4clMeti-E) ; (9) 14a-hydroxy-hydrocortisone; (10) $\Delta^{4}$ androstene-3,17-dione; (11) $11 \beta \mathrm{OH}-\Delta^{4}$-androstene-3, 17 dione ; (12) $11 \beta \mathrm{OH}-\Delta^{1,4}$-androstadiene-3,17-dione ; (13) 1-dehydro-testosterone (Meti-testosterone); (14) $9 \alpha \mathrm{F}$, $11 \beta \mathrm{OH}-\Delta^{1,4}$-androstadiene-3,7-dione ; (15) $9 \alpha \mathrm{F}, 11 \beta \mathrm{OH}$, $17 \alpha$-methyl-testosterone ; (16) 1-dehydro-17 $\alpha$-methyl-testosterone (methyl meti-testosterone).

In the 21-carbon adrenocortical series $11 \beta$-hydroxylation accompanies anti-anabolic activities. These activities are enhanced by further chemical alteration such as 1,2-

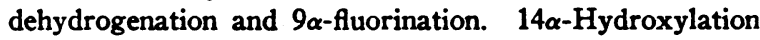
abolishes anti-anabolic activities. 4-Chlorination decreases anti-anabolic activities. 1,2-Dehydrogenation partially activates inactive $C_{\infty}$ reduced adrenocortical steroid. In the 21-carbon series, 1,2-dehydrogenation is associated with: (1) decreased tubular reabsorption of sodium; (2) increased proportion of unconjugated 21-carbon metabolites; (3) markedly decreased conversion to 17-ketosteroids; (4) altering principal pathways of parent steroids' metabolism indicating cleavage of the dihydroxyacetone side chain preceding ring $A$ reduction.

In the 19-carbon anabolic series, 1,2-dehydrogenation decreases anabolic activities. 11 $\beta$-hydroxylation markedly decreases anabolic activities. 1,2-Dehydrogenation, $9 \alpha$ fluorination and $11 \beta$-hydroxylation together abolish anabolic activities. 1,2-Dehydrogenation markedly decreases excretion of 17-ketosteroids, contrasting sharply with the metabolism of 19-carbon parent steroids.

An 11 oxygen atom is essential for anti-anabolic activities in the 21-carbon series. Enhancement of these activities by 1,2-dehydrogenation and $9 \alpha$-fluorination depends on the presence of an 11 oxygen atom. The anabolic activities of 19-carbon steroids are markedly decreased by $11 \beta$-hydroxylation. Thus the presence or absence of an oxygen atom at $C_{11}$ in 19-carbon and 21-carbon steroids largely determines the anabolic or anti-anabolic activities of these steroids.

\section{The Influence of Arm Circulation on Clinical Circula- tion-Time Measurements. Solbert Permutt, Marian Isaacs, and Edvardas Varnauskas, Baltimore, Md. (Introduced by Richard L. Riley).}

When an indicator is injected in an antecubital vein and sampled in the contralateral brachial artery, the mean circulation time (C.T.) is significantly longer than when indicator is injected through a catheter in the subclavian vein. We consider this difference a measure of arm C.T. This difference is greatly diminished if the antecubital injection is made in an arm where blood flow is markedly increased by reactive hyperemia. Under these circumstances, arm C.T. is approximated by the difference in C.T. before and during hyperemia ( $\Delta$ C.T.). To evaluate the influence of arm C.T. on total C.T. from elbow to tongue, we have measured $\Delta$ C.T. in 12 normal subjects and 14 patients with varying degrees of congestive heart failure. Three $\mathrm{ml}$. of Decholin were in- 
jected in an antecubital vein, first, in the routine manner and then three seconds following the release of a blood pressure cuff, proximal to the needle, inflated above 250 $\mathrm{mm}$. $\mathrm{Hg}$ for five minutes. In normal subjects, average total C.T. was 15.2 (S.D. 3.1) and $\Delta$ C.T. was 4.2 (S.D. 3.0). C.T. during hyperemia, which averaged 11.0 (S.D. 1.4), showed significantly less variation than total C.T. or $\Delta$ C.T., suggesting that variations in total C.T. of normal subjects are due chiefly to variations in arm C.T. In cardiac subjects, average total C.T. was 31.9 (S.D. 9.0) and $\Delta$ C.T. was 8.1 (S.D. 5.4), both values being significantly greater than in the normal subjects, suggesting that arm C.T. in cardiac patients tends to be prolonged in proportion to the increase in total C.T. The increased variability of total C.T. in cardiac patients appears to be due to variations in both arm and central C.T.

Studies in Adrenocortical Function in Liver Disease. Ralph E. Peterson, Bethesda, Md. (Introduced by Harry Eagle).

Adrenal function in liver disease was studied by determination of rate of metabolism and rate of synthesis of hydrocortisone. Four patients with cirrhosis and one patient with acute viral hepatitis were studied. Patients with liver disease showed a 2 to 6-fold delay in rate of biotransformation of infused hydrocortisone. These same patients were able to metabolize the initial reduction product of hydrocortisone (dihydrocortisone) to tetrahydrohydrocortisone at the normal rate. They also showed no significant impairment in their ability to metabolize tetrahydrohydrocortisone to its glucuronide. The miscible pools and plasma hydrocortisone levels were normal or only slightly increased in the presence of low levels of urinary corticosteroids and 17-ketosteroids. The turnover rates of hydrocortisone were found to be decreased, 20 to 50 per cent of normal, when conventional tracer techniques, using hydrocortisone-4- $\mathrm{C}^{14}$, were applied. These results are consistent with the interpretation that in liver disease there is a selective defect in the liver enzyme system (dihydrohydrocortisone dehydrogenase) necessary for the conversion of hydrocortisone to dihydrocortisone. This defect might be expected to cause an elevation of the plasma hydrocortisone level with a resultant suppression of ACTH production and a decreased secretion of hydrocortisone by the adrenal.

Serial Renal Biopsies in Toxemia of Pregnancy. VICtoR E. Pollak, John B. Nettles, Conrad L. Pirani, Robert M. Kark,* and Sjoerd L. Bonting, Chicago, III.

Nutritional deficiency, endocrine overactivity, and neurogenic stimuli from the gravid uterus are current theories of the etiology of pre-eclampsia and eclampsia. Percutaneous renal biopsies were made in 35 women diagnosed "toxemia of pregnancy." In 27, unique glomerular lesions were found; when serial biopsies were made after confinement, the lesions had disappeared. These characteristic pre-eclamptic lesions were readily distinguished from lesions in five cases of toxemia with es- sential hypertension, and from other types of renal disease in pregnancy (three cases).

In pre-eclampsia, blood corticoid and urinary aldosterone levels are elevated, but the renal lesions were unlike those we have seen in biopsies from patients with Cushing's syndrome, pharmacologic hypercortisonism, and primary hyperaldosteronism. Although pre-eclamptic women have abnormal pyridoxal metabolism, the renal lesions did not resemble those of experimental pyridoxine deficiency. To determine whether vasospastic agents capable of passing the placental barrier might cause the maternal renal lesion, the kidneys of ten fetuses born to pre-eclamptic mothers were examined; no abnormality was observed.

The characteristic lesion in pre-eclampsia is not a membranous glomerulonephritis as others have stated. In our material all glomeruli in the sections were affected in like degree, being larger than normal and almost completely bloodless. Details of the abnormalities became evident with the oil immersion lens. The observations were confirmed with periodic-acid-Schiff, Mallory-azan, van Gieson, reticulin, and fat stains. The glomerular basement membrane was thin and delicate; the swelling of the tuft was due to gross edema of the cytoplasm of endothelial and epithelial cells. Preliminary measurements of glomerular water (Lowry, J. Histochem., 1953, 1, 420) confirmed that these glomeruli have an increased water content $(0.105 \mathrm{Kg}$. dry weight per liter; normal $0.130 \mathrm{Kg}$. dry weight per liter).

These studies appear to support the concept that the renal lesion of pre-eclampsia develops as a result of neurogenic stimuli from the gravid uterus.

\section{Relation of Filtered Bicarbonate to Bicarbonate Excre- tion During Diamox Administration. RICHARD M. Portwood, Donald W. Seldin,* Floyd C. Rector, Jr., and Robert Cade, Dallas, Tex.}

The relationship between plasma $\left[\mathrm{HCO}_{3}{ }^{-}\right]$and $\mathrm{HCO}_{3}{ }^{-}$ excretion during Diamox administration was explored in normal subjects during maximum water diuresis in whom sharp reductions in plasma $\left[\mathrm{HCO}_{3}^{-}\right]$and filtered $\mathrm{HCO}_{8}^{-}$were produced by hyperventilation. During periods of comparable water, buffer, and $\mathrm{HCO}_{3}^{-}$excretion, urine $\mathrm{pCO}_{2}$-plasma $\mathrm{pCO}_{2}$ was used as an approximate index of distal tubular hydrogen secretion.

Hyperventilation depressed plasma $\left[\mathrm{HCO}_{3}^{-}\right]$from control values of $23 \pm 1$ to $16 \pm 1 \mathrm{mEq}$. per L., with comparable falls in filtered $\mathrm{HCO}_{3}^{-}$. $\mathrm{HCO}_{3}^{-}$excretion rose slightly from $15 \pm 9$ to $29 \pm 14 \mu \mathrm{Eq}$. per min. Urine $\mathrm{pCO}_{2}$ decreased from $43 \pm 7$ to $30 \pm 7 \mathrm{~mm}$. $\mathrm{Hg}$. Urine pCOs-plasma $\mathrm{pCO}$ increased from 8 to $14 \mathrm{~mm}$. $\mathrm{Hg}$.

Diamox plus hyperventilation depressed plasma [ $\mathrm{HCO}_{3}^{-}$] and filtered $\mathrm{HCO}_{8}^{-}$to the same extent as hyperventilation alone, but markedly accelerated $\mathrm{HCO}_{3}^{-}$ excretion to $544 \pm 150 \mu \mathrm{Eq}$. per min. Urine $\mathrm{pCO}_{2}$ was only slightly elevated to $50 \pm 5 \mathrm{~mm}$. $\mathrm{Hg}$ with a modest increase in urine pCOr-plasma $\mathrm{pCO}_{2}$ to $34 \mathrm{~mm}$. $\mathrm{Hg}$.

Diamox alone did not depress plasma $\left[\mathrm{HCO}_{8}^{-}\right]$or filtered $\mathrm{HCO}_{3}^{-}$appreciably but augmented $\mathrm{HCO}_{8}^{-}$excre- 
tion to no greater extent than did hyperventilation plus Diamox. However, urine $\mathrm{pCO}$-plasma $\mathrm{pCO}$, increased strikingly to $48 \mathrm{~mm}$. $\mathrm{Hg}$.

These data suggest that $\mathrm{HCO}_{3}^{-}$diuresis produced by Diamox ${ }^{(1)}$ is dependent, not only on filtered $\mathrm{HCO}_{3}^{-}$, but also on the rate of $\mathrm{H}^{+}$secretion as influenced by blood $\mathrm{pCO}_{2}$ and carbonic anhydrase activity. With hyperventilation alone, diminished $\mathrm{CO}_{3}$ available for $\mathrm{H}^{+}$production will reduce $\mathrm{H}^{+}$secretion only modestly, since the carbonic anhydrase system is intact; $\mathrm{HCO}_{8}^{-}$diuresis is therefore small. During hyperventilation plus Diamox the low tissue $\mathrm{pCO}_{2}$ during inhibition of carbonic anhydrase so impairs the uncatalyzed reaction that even small filtered loads cannot be reabsorbed, resulting in marked $\mathrm{HCO}_{3}^{-}$ diuresis of as much as $\mathbf{4 0}$ per cent of the filtered load. The diminished $\mathrm{H}^{+}$secretion under these circumstances is attested by the lower urine $\mathrm{pCO}_{2}$-serum $\mathrm{pCO}_{2}$ as compared with comparable $\mathrm{HCO}_{3}^{-}$diuresis with Diamox alone.

The Hypoglycemic Properties of Dimercaprol (BAL). Thaddeus E. Prout, John A. Weaver, George W. Scott, and Samuel P. Asper, JR,,* Baltimore, Md.

A study of the effect of dimercaprol (BAL) on carbohydrate metabolism was made in ten patients with diabetes mellitus and in laboratory animals. The observation of Butterfield (Lancet, 1955, 1, 489) that BAL reduces fasting blood sugar and glycosuria in diabetics was confirmed in two of seven diabetics with severe disease. In one severe diabetic, receiving constant diet and insulin, the fasting blood sugar decreased from 340 to $37 \mathrm{mg}$. per cent with symptoms of hypoglycemia on the third day of BAL administration. Insulin sensitivity was increased by BAL in three patients with moderate diabetes but was unaffected in three of those with severe disease. Irritation at sites of BAL injection limited clinical use.

BAL was administered intravenously to normal rabbits in increasing quantities, 0.06 to $0.36 \mathrm{mM}$ per $\mathrm{Kg}$. alone, or after prior mixing with insulin, 0.6 unit per $\mathrm{Kg}$. The insulin effect was attenuated in a step-wise fashion with increasing quantities of BAL and abolished by BAL 0.18 $\mathrm{mM}$ per $\mathrm{Kg}$. This latter quantity of BAL given alone, however, caused a 28 per cent decrease in blood sugar at three hours. In the normal rabbit BAL $0.18 \mathrm{mM}$ per $\mathrm{Kg}$. did not alter the rate of glucose disappearance following an intravenous glucose load, the rate of disappearance of labeled insulin from serum or the degree of insulin sensitivity. In adrenalectomized rats hypoglycemia following BAL was profound, and frequently fatal, but was diminished by cortisone. Increasing BAL to $0.36 \mathrm{mM}$ per $\mathrm{Kg}$., with or without insulin, produced hyperglycemia lasting more than three hours; the hyperglycemia appears to be an epinephrine effect since it was abolished by prior injection of ergotamine tartrate $1.0 \mathrm{mg}$. per $\mathrm{Kg}$. in rabbits or by adrenal demedullation in rats.

The mechanism producing hypoglycemia is obscure. These studies demonstrate that BAL has a hypoglycemic effect in some diabetic patients and outline conditions which influence the response in laboratory animals.

\section{Preparation of Growth Hormone from Human Pituitaries.} M. S. RABEN,* Boston, Mass.

Human pituitaries were removed postmortem and stored in acetone; weeks to months later, the pituitaries were homogenized, washed further with acetone and dried in vacuum. Growth hormone was extracted and purified from the acetone-dried powder by the method originally devised for porcine pituitaries. The powder was extracted with glacial acetic acid at $70^{\circ} \mathrm{C}$, an acetone precipitate discarded and the active fraction precipitated with ether. Corticotropin and intermedin were removed with oxycellulose, a precipitate at $\mathrm{pH} 8.5$ was removed and growth hormone was brought down with the addition of alcohol. The yields from batches of $11.25 \mathrm{grams}$ and 24 grams of powder were $350 \mathrm{mgs}$. and $1,100 \mathrm{mgs}$, or two and three times the usual yield from pig. As assayed in hypophysectomized rats, the human and porcine preparations were of similar activity, with the human perhaps slightly more potent. Evidence of anabolic effect in man was obtained in five patients in four different institutions by $J$. Beck and Venning, Pearson, Henneman, R. N. Beck and Raben and their respective co-workers. Growth hormone was also prepared by the same method from monkey pituitaries and found active in the one patient tested by Beck and Venning. The effective dose appears to be small enough (less than 0.1 mg. per kilogram) to make human autopsy pituitaries a practical source of growth hormone for clinical use, since preparations from slaughterhouse animal pituitaries have been generally ineffective in man. A fortunate feature of this method of preparation of growth hormone as applied to human pituitaries of indeterminate origin is the bactericidal and viricidal action provided by the use of acetone, ether and hot glacial acetic acid.

Kinetic Spectrophotometric Studies of a DPNH-Succinate Cytochrome $c$ Reductase from Heart Muscle. MURRAY RABINowitz, Ronatd Esterbroor, and BENEDEtTo DeBernard, Philadelphia, $\mathrm{Pa}$. and Madison, Wisc. (Introduced by William P. Chapman).

The synthesis of ATP, the source of energy for cardiac muscle contraction, is largely coupled to electron transport from substrate to oxygen. To gain further information about this process, the electron transfer system of beef heart muscle has been fractionated into simpler units and the function of these units examined. By cholate- $\left(\mathrm{NH}_{4}\right)_{2} \mathrm{SO}_{4}$ fractionation a particulate preparation has been obtained which contains as functioning components DPNH and succinic dehydrogenases, flavoprotein and cytochromes $b$ and $c_{1}$; cytochrome oxidase and cytochrome $c$ have been completely removed. Kinetic studies have been performed on this DPNH-succinate cytochrome $c$ reductase using Chance's rapidly recording dual monochromator spectrophotometer.

It was demonstrated that the flavoproteins associated with DPNH and succinate dehydrogenases were different. Difference spectra of these two flavoproteins were individually recorded.

The velocity reduction of cytochrome $c_{1}$, a newly es- 
tablished component of the electron transfer system, was several times greater than that of cytochrome $b$, but only one-third to one-fifth that of added excess cytochrome $c$. Cytochrome $c$ addition failed to increase reduction velocities of cytochromes $b$ or $c_{1}$. Flavoproteins were reduced at rates similar to that of added cytochrome $c$. These observations indicate direct flavoprotein interreaction with all three cytochromes in this preparation. The pattern of cytochrome reduction was identical with DPNH or succinate. The direct flavoprotein-cytochrome $c$ pathway was inhibited by Antimycin A, in contrast to the Antimycin insensitivity of soluble DPNH cytochrome $c$ reductase. Antimycin A decreased reduction velocities of cytochromes $c$ and $c_{1}$, but markedly increased the reduction rate of cytochrome $b$. Amytal inhibited the rates of reduction of all cytochromes by DPNH, but left unaffected flavoprotein or ferricyanide reduction. Succinate oxidation was unaffected.

Diffusion Characteristics of the Pulmonary Membrane and Capillary Bed in Various Diseases of the Lungs and Cardio-Vascular System. John Rankin, Ronald S. McNeIll, and Robert E. Forster,* Philadelphia, Pa.

A method has recently been developed for calculating the diffusing capacity of the pulmonary membrane (Du) and the average amount of hemoglobin in the pulmonary capillary bed $(\mathrm{PCH})$ from a) the diffusing capacity of the lung for $\mathrm{CO}\left(\mathrm{D}_{\mathrm{L}}\right.$, which includes diffusion within the capillary as well as across the membrane) and b) the in vitro rate of combination of $\mathrm{CO}$ with intracorpuscular hemoglobin measured at several different alveolar $\mathrm{O}_{2}$ tensions from 100 to $650 \mathrm{~mm}$. $\mathrm{Hg}$. If the hematocrit in the pulmonary capillaries is known, the pulmonary capillary blood volume $\left(C_{e}\right)$ can be calculated from $P C H$. Measuring $D_{L}$ at rest by a breath-holding technique, we have obtained $D_{\mathbf{x}}$ and $\mathrm{PCH}$ in 9 normal subjects and 20 patients with anemia, polycythemia, or cardio-pulmonary disease. The results are summarized as follows. In normal subjects $D_{\mathbf{M}}$ was about twice $D_{\mathbf{L}}$, the latter averaging $32 \mathrm{ml}$. per min. per $\mathrm{mm}$. $\mathrm{Hg} ; \mathrm{V}_{\mathrm{e}}$ averaged 98 $\mathrm{ml}$. on the basis of the peripheral hematocrit. In patients with diffuse pulmonary fibrosis, $D_{L}$ was markedly reduced, owing in the main to a decrease in $D_{\mathbf{M}}$, and to a lesser decrease in $\mathrm{PCH}$ in some instances. In patients with pulmonary vascular congestion as a result of heart disease, $\mathrm{PCH}$ was markedly increased, while $\mathrm{D}_{\mathbf{x}}$ was normal or slightly decreased. In 4 out of 5 patients with anemia and no evidence of pulmonary disease, $\mathrm{D}_{\mathbf{L}}$ was reduced and $\mathrm{PCH}$ was markedly reduced. In 2 of these patients $D_{\mathbf{u}}$ was also markedly reduced. These values tended towards normal as the anemia was corrected by therapy. $\mathrm{PCH}$ was increased in polycythemia.

Increased Susceptibility to Viral Infection Induced Experimentally by Psychophysiologic Stress. A. F. RAsMUSSEN, Jr.,* James T. Marsh, and Norman Q. BrmL, Los Angeles, Calif.

A variety of factors other than specific immunity modifies host response to infectious agents, protecting against repeated exposure to potentially dangerous agents on one hand, and subtly converting innocuous exposures and inapparent infection into overt disease on the other. Psychologic stress is often proposed as one of the factors predisposing to overt infection. Patients with histories of repeated infections have shown increased resistance following psychotherapy. Clinical evidence supporting an etiologic role for emotional stress in recurrent herpes simplex is particularly impressive. As the initial step in an attempt at elucidation of psychologically induced changes in resistance, an experimental system demonstrating stress-induced increases in susceptibility of mice to infection with the virus of herpes simplex is described. The system follows techniques widely employed in experimental psychology for the production of behavioral changes in animals.

Avoidance-learning stress was produced by subjecting mice to repeated cycles of, first, warning stimuli, a bright light and buzzer, followed by electric shock, a painful stimulus. Animals were able to avoid the painful stimulus by jumping a barrier immediately after the warning stimuli and animals learned to avoid the shock fifty to eighty per cent of the time after two or three days. Restraint-stress was produced by confining mice in snugly wrapped wire mesh blankets. Mice subjected to either form of stress for periods of six hours daily for two to four weeks showed increased susceptibility to intraperitoneal inoculation with the virus of herpes simplex. The increase in susceptibility was manifested by statistically significant increases in mortality in stressed as compared to control animals, and by a trend toward shorter incubation periods in stressed animals.

\section{An Hepatic Mechanism for Tolbutamide Hypoglycemia in Animal and Human Subjects. LILLIAN RECANT* and George L. Fischer, St. Louis, Mo.}

Hypoglycemia may result from decreased hepatic release of glucose, increased peripheral utilization of glucose, or a combination of the two processes. In an effort to define the mechanisms involved in tolbutamideinduced hypoglycemia, the following experimental observations were made.

Determinations of liver slice glucose release and diaphragm muscle glucose uptake were made on isolated liver and muscle removed from rats, at 15, 30, 45 and 60 minutes following I.P. tolbutamide. The time-curve of blood sugar fall correlated directly with a decrease in liver slice glucose release. No evidence of enhanced glucose uptake in muscle was found.

Human subjects were studied, utilizing the technique of hepatic venous catheterization. Measurements of peripheral arterio-venous sugars were also made. Following I.V. tolbutamide, a fall in hepatic glucose release associated with a 20 to 60 per cent increase in hepatic vein pyruvate was observed. No increase in peripheral glucose utilization could be demonstrated.

These findings suggested that hypoglycemia resulted from a retention of carbohydrate by liver with an as- 
sociated increase in hepatic glucose utilization, as evidenced by the rise in pyruvate. The following study further supports this hypothesis. In vitro $\mathrm{C}^{14}$-glycine incorporation into liver slice protein was studied in 24 starved control rats and in 23 starved rats pretreated with tolbutamide. The latter group showed a 54 per cent increase in incorporation rate. (Specific Activity increased from 65.5 to 101.3 counts per min. per $100 \gamma$ protein.) It has been shown previously that $\mathrm{C}^{16}$-glycine incorporation depends upon active glycolysis and is known to be impaired in starvation and diabetes and to be restored by glucose and insulin.

The observations made are consistent with an hepatic insulin-like action following tolbutamide. Preliminary studies indicate that tolbutamide increases "insulin activity" in dog pancreatic blood. The absence of peripheral "insulin activity" following tolbutamide requires explanation and will be discussed.

\section{Effect of Dialysis and Purine Ribosides Upon the Anemia} of Uremia. Searle B. ReEs, Walter G. Scheitlin, John C. Pond, Thomas J. McManus, Warren R. GuILd, and JoHn P. MerriLl,* Boston, Mass.

Erythrocytes from uremic patients live a normal life span in healthy recipients despite increased random cell destruction in their host. We are, therefore, investigating remediable cellular and extracellular factors contributing to the anemia of uremia. Ten chronically ill uremic patients manifesting varying degrees of renal decompensation were selected for hemodialysis. All demonstrated a normochromic, normocytic anemia, hyperosmolarity, compensated metabolic acidosis, azotemia, electrolyte imbalance, and an anion deficit.

Pre-dialysis reticulocyte counts and $\mathrm{Cr}^{\mathrm{st}}$ cell volume and survival studies revealed increased red cell destruction and inadequate erythropoiesis to compensate for this deficit. In vitro erythrocyte equilibrations revealed inability to maintain normal phosphate and potassium balance. Net resynthesis of phosphate esters and steady state potassium flux could be maintained when uremic cells were equilibrated in compatible fresh plasma fortified with a purine riboside, inosine. Uremic cells metabolize purine riboside via phosphorolysis and extra-glycolytic pathways as effectively as normal human erythrocytes.

All patients showed clinical improvement following dialyses calculated not to lower the blood urea nitrogen but which removed anion deficits. Although clinical improvement occurred in 2 to 3 days, red cell survival and in vitro equilibration characteristics did not return to. nearly normal until 4 to 6 days after dialysis.

Administration of the purine riboside, inosine, partially reversed a biochemical lesion observed in uremic erythrocytes in vitro. These studies have been extended in vivo. Two patients received inosine intravenously. Within one hour the erythrocytes demonstrated normal potassium and phosphate ester contents.
Electrolyte Balance and Acid-Base Metabolism in Primary Aldosteronism. ARnold S. ReLMAN * and WILliam B. Schwartz,* Boston, Mass.

In two patients with severe primary aldosteronism, studies were carried out to define the nature of the disturbances in electrolyte and acid-base physiology.

1. As might be anticipated from the known influence of sodium intake on the kaliuretic action of mineralocorticoids, a significant negative balance of potassium occurred only when sodium was ingested. On a normalpotassium, low-sodium intake, potassium balance was slightly positive. Although urine concentrations were sometimes 5 to $6 \mathrm{mEq}$. per L. (daily volume 5 to 6 liters), potassium excretion was always inappropriately high compared to the low plasma levels. With potassium loading, one patient continuously retained a portion of the load until the $1,000-\mathrm{mEq}$. deficit was corrected. In the other patient, even on a low-sodium diet containing $250 \mathrm{mEq}$. of potassium, there was only a transient period of retention.

2. The urine was always relatively alkaline, and fourhour collections through the day showed little or none of the usual diurnal fluctuations in $\mathrm{pH}$ or acid excretion. With widely varied urine flows, bicarbonate excretion was nearly a direct function of flow. Ammonium and potassium concentrations varied widely, but concordantly, independently of $\mathrm{pH}$ and roughly inversely with flow. There was no inherent defect in the acidifying mechanism, however, since urine pH's as low as 5.1 to 5.2 occurred during sulfate loading.

3. There was no "paradoxical" response to cortisol or DOCA.

4. Correction of alkalosis at the beginning of the potassium-loading period was associated with a further rise in urine $\mathrm{pH}$ and a diuresis of bicarbonate, large enough in one case to explain the drop in serum bicarbonate.

5. In one patient, following removal of the adrenal tumor, a syndrome of hypoaldosteronism appeared, with persistance of defective sodium conservation and hyperkalemia for several months.

\section{Physiological Implications of Thyroxine-Binding by Se- rum Proteins. JACOB RoBBINS and J. E. RALL,*} Bethesda, Md.

Thyroxine in human serum is reversibly bound to an alpha globulin (TBP) and, in lesser degree, to albumin. Free (unbound) thyroxine is not directly measurable by present techniques. Utilizing thyroxine- $\mathrm{I}^{120}$ and reverse flow zone electrophoresis, and data in the literature for bovine serum albumin, one may calculate the association constant for thyroxine and TBP $\left(8 \times 10^{\circ}\right)$ and the concentration of free thyroxine in serum (mean normal, $\left.6 \times 10^{-11} \mathrm{M}\right)$. These values, although approximate, appear valid for intercomparisons.

Free thyroxine was calculated in two groups of patients with paradoxical protein-bound iodine (PBI) levels. In euthyroid pregnant women, elevated PBI was 
accompanied by an increase in concentration of TBP. The resulting free thyroxine was normal (3.5 to $6.0 \times$ $\left.10^{-11} \mathrm{M}\right)$. Nephrotic subjects with low PBI had low TBP levels and, in all but one, free thyroxine was normal or elevated ( 3.1 to $\left.32 \times 10^{-11} \mathrm{M}\right)$. In contrast, hypothyroid subjects had an elevated TBP, so that free thyroxine was relatively lower than PBI. In hyperthyroidism, TBP was unchanged; the increase in PBI was, therefore, accompanied by a relatively greater increase in free thyroxine $\left(21\right.$ to $\left.71 \times 10^{-11} \mathrm{M}\right)$. Thus, free thyroxine was a satisfactory index of clinical thyroid status in hypothyroidism and hyperthyroidism. In pregnancy and nephrosis, it appeared to be a better index than PBI.

To test whether the metabolism of thyroxine might also be related to free thyroxine, data from Berson and Yalow were used. Free serum thyroxine was calculated from the PBI and compared with thyroxine degradation. A linear relationship between these two variables was found.

It appears probable, therefore, that thyroxine bound to serum protein is inactive and that the level of free thyroxine in serum is one of the factors which govern the general metabolic effects of thyroxine and its rate of degradation.

The Nature of the Respiratory Acidosis of Sleep and of the Respiratory Alkalosis of Hepatic Coma. Eugene D. Robin, Robert D. Whaley, Charles H. Crump, and David M. Travis, Boston, Mass. (Introduced by Eugene C. Eppinger).

Carbon dioxide retention is known to accompany sleep, but the magnitude and mechanism of this retention have not been determined. A study of this phenomenon was, therefore, undertaken in fourteen normal subjects, in four patients with chronic hypercapnia and in five patients with hepatic coma.

Normal subjects during sleep show a rise in alveolar carbon dioxide tension to as high as $52 \mathrm{~mm}$. $\mathrm{Hg}$. There is a fall in alveolar ventilation, oxygen consumption and carbon dioxide production. Alterations in respiratory rhythm, including cyclic breathing of the Cheyne-Stokes variety, were observed in six of the fourteen normal subjects. The ventilatory response to carbon dioxide is depressed during sleep, falling from 1.3 to 0.3 liter per minute per $\mathrm{mm}$. $\mathrm{Hg}$. Onset and reversal of this depressed response occur almost simultaneously with sleep and wakefulness, respectively.

Chronic hypercapnia is associated with depression of the ventilatory response to carbon dioxide in a waking patient. This depression becomes more profound during sleep and this results in rises of alveolar carbon dioxide tension to as high as $70 \mathrm{~mm}$. Hg. Cheyne-Stokes breathing during sleep was seen in three of four patients with chronic hypercapnia.

Hepatic coma is associated with alveolar hyperventilation and hypocapnia which are independent of the patient's state of consciousness. Even in the presence of this increased ventilation the respiratory sensitivity to carbon dioxide is not increased; indeed, in deep coma it is depressed. Thus the respiratory alkalosis of hepatic coma appears to result from the stimulation of the respiratory control system by factors acting independently of the carbon dioxide-pH system.

These data demonstrate that carbon dioxide retention in sleep is related to a depression of respiratory center sensitivity. The rapid development of this depression suggests that it is mediated neurogenically.

Pulmonary Vascular Responses to Serotonin and the Effects of Serotonin Antagonists. JoH N C. RosE, Washington, D. C. (Introduced by Edward D. Freis). In intact animals, 5-hydroxytryptamine (serotonin) elevates pulmonary vascular resistance. Pulmonary hemodynamics are influenced by extrapulmonary factors (right ventricular output, left atrial pressure, blood volume redistribution) and altered ventilation. The following experiments showed that the serotonin effect is due to direct pulmonary vasoconstriction.

A diaphragm pump of controlled output was substituted for the left ventricle in 16 dogs. Blood was drained from the left auricle to a reservoir, from which it was pumped to a $T$-tube in the thoracic aorta. Left ventricular bypass was complete. The right ventricle continued functioning normally.

As serotonin ( 2 to $40 \mathrm{mcg}$. per $\mathrm{Kg}$.) was injected into the pulmonary artery, the tube draining the left auricle was temporarily diverted to a second reservoir. During this period, the circulation was maintained by blood pumped from the previously-filled main reservoir. The drug traversed only the lungs. When the main pump reservoir was nearly depleted, the auricular tube was returned to its original position and the blood in the temporary container was returned to the circulation.

Confined to the pulmonary vasculature, serotonin caused immediate pulmonary hypertension and decreased blood flow. Precisely similar pressure and flow changes followed the same dose when given at 5-minute intervals. No substance studied except serotonin produced pulmonary vascular effects in doses insufficient to affect systemic arterial pressure.

Systemic vascular reflexes were not initiated by serotonin in the lungs. (These reflexes are easily demonstrated in this preparation using veratrum.)

Reproducibility of pulmonary vascular response permitted evaluation of several "antiserotonin" substances. Phenothiazine (Phenergan 8 ), 0.6 to $1.0 \mathrm{mg}$. per $\mathrm{Kg}$., completely abolished pulmonary vascular response to serotonin. No other antihistamine studied had serotoninblocking properties. Antiserotonin effect of phenothiazine was more potent than that of the serotonin anti-metabolite, BAS. LSD-25 and its 2-bromo derivative abolished pulmonary vascular effects of serotonin in slightly smaller doses than phenothiazine.

Zone Electrophoretic Studies of Serum Alkaline Phosphatase Activity. I. N. RosenberG,* Boston, Mass.

Samples of serum of abnormally high alkaline phosphatase activity obtained from patients suffering from 
Paget's disease and from obstructive and infiltrative disease of the liver and bile ducts were subjected to electrophoresis in starch blocks. Sodium barbital buffer, $\mathrm{pH}$ 8.6, ionic strength 0.1 was used, and electrophoresis was conducted at $5^{\circ} \mathrm{C}$ for 48 hours at a potential difference of approximately 6 volts per centimeter. After electrophoresis, the block was divided transversely in 5-millimeter sections and the protein concentration and alkaline phosphatase activity of the filtered aqueous eluates of these sections were determined. Hydrolysis of p-nitrophenylphosphate, $\mathrm{pH} 10.3$, was used to measure phosphatase activity.

In Paget's disease (seven cases) the serum electrophoretic pattern indicated nearly all the alkaline phosphatase activity to be in a single band whose peak was at, or slightly behind, the alpha-2 globulin peak; less than 5 per cent of the activity was in the alpha-1 globulin zone. By contrast, in 13 cases of obstructive jaundice and hepatic cancer, the serum phosphatase pattern showed at least two bands; the larger corresponded to the band observed in the Paget's cases, while the smaller, representing 6 to 19 per cent of the total activity, was of greater mobility, with a peak in the alpha-1 globulin region. A broad band of phosphatase activity with mobility approximately that of beta-globulin was noted in two cases of biliary cirrhosis, and a poorly-resolved zone of activity in this region was noted in several other cases of hepatic disease. The electrophoretic serum alkaline phosphatase patterns of four normal subjects qualitatively resembled those of Paget's disease. The results suggest that there may be qualitative differences in the patterns of the serum alkaline phosphatase activity in various clinical states.

The Use of Albumin-Heparin Infusion as an Anti-Lipemic Agent. Ray H. Rosenman,* San Francisco, Calif.

Lipemia clearing is preceded by hydrolysis of triglycerides, a reaction catalyzed by lipoprotein lipase and controlled by albumin since released fatty acids are complexed with albumin. Since such hydrolysis appears requisite for plasma clearance of triglycerides, the following studies were made of the possible use of albuminheparin infusion as an anti-lipemic agent.

Hyperlipemia was produced in rats by inducing nephrosis, by Triton injection, and by injection of hyperlipemic rabbit serum. The rats were variously infused with serum albumin containing heparin, or with only albumin, heparin, or saline. The albumin-heparin infusion induced a marked fall of plasma triglycerides, phospholipids, and cholesterol in the nephrotic and Tritonized rats, and accelerated clearance of excess plasma lipids in those injected with lipemic rabbit serum, in each instance exceeding the response induced by separate injections of heparin or albumin. The differences weren't ascribable to variations of induced hemodilution. The amount of cleared lipids was proportional to both the magnitude of the hyperlipemia and to the amount of injected albumin, suggesting that the lipid-clearing mechanism obeys mass action principles.
Although the heparin-activated reaction appears fundamentally concerned with hydrolysis and clearance of plasma triglycerides, it is possible that it also is directly concerned with clearance of excess phospholipids and cholesterol from the plasma. Thus it was found that albumin-heparin injection also accelerated clearance of excess phospholipid and cholesterol from plasma of rats injected with such lipids contained in serum of bile duct-ligated rats, which is not turbid and contains no excess triglycerides. In contrast, no fall of plasma lipids occurred in normal, fasting rats infused with albuminheparin.

Thus albumin-heparin infusion is an effective antilipemic agent and heparin-activated mechanisms may be concerned with phospholipid and cholesterol as well as triglycerides.

Observations on the Pathogenesis of Juvenile Pernicious Anemia. Cyrus E. RubIN, Seattle, Wash. (Introduced by Wade Volwiler).

This is a pathogenetic study of eight well-authenticated cases of juvenile pernicious anemia, in whom the stomachs uniquely secrete $\mathrm{HCl}$ without producing intrinsic factor. This contrasts with the invariable achylia of adult p.a., a finding which has led to the presumption that gastric fundal atrophy causes defective intrinsic factor production.

The data suggest that juvenile p.a. constitutes the homozygous state for adult Addisonian p.a. Six patients had siblings with the same disease; three had parental consanguinity and others had a suggestive family history of adult p.a.

The ages ranged from 3.6 to 24 years in these eight patients. Seven patients now secrete $\mathrm{HCl}$ without stimulation although many were previously shown to have histamine-fast achlorhydria during repeated exacerbations. Treatment began before two years of age in six patients. The only patient with permanent achylia was initially treated at 12 years, the latest institution of therapy in this series. Considering the intrinsic factor deficiency in the acid secretors, the finding in one-third of them of pepsin levels in the achylic range was of interest.

Histologically, suction biopsies revealed ample parietal and chief cells in the seven acid secretors, unlike the extreme glandular atrophy observed in the child last treated and in all adult p.a. Minimal residual abnormalities possibly reflected gastritis during past exacerbations. The one patient biopsied during an exacerbation had hypochlorhydria and moderate gastritis. Large gastric columnar cells were exfoliated twice: in the achylic and in a patient with a previous, severe, protracted exacerbation.

Intestinal vitamin $B_{12}$ malabsorption in addition to intrinsic factor deficiency was present in four patients.

These studies of earliest p.a. make it apparent that the essential hereditary feature is diminished production of intrinsic factor. Protracted subclinical vitamin $B_{12}$ deficiency may then produce secondary irreversible atrophic 
gastritis with its potentialities for polyps and carcinomas as frequently seen in adults.

Effect of the Type of Urinary Solute on the Process of Urine Concentration. M. E. RubinI, W. B. Blythe, and W. H. MERoney, Washington, D. C. (Introduced by Laurence $\mathrm{H}$. Kyle).

The maximal osmolar concentration of urine attainable with dehydration and exogenous $A D H$ is significantly increased following brief high-protein feeding. This effect of diet is independent of the total quantity of solutes ingested, and the degree of antecedent dehydration. Possible causes for the increased concentrating ability include adaptive augmentation of the tubular capacity to absorb water, or some property peculiar to the type of solute excreted.

Experiments were designed to exclude adaptation as a principal factor, and to test the hypothesis that urea may allow greater water reabsorption than other urinary solutes. Acute studies were performed in patients with hyposthenuria, normal subjects, and dogs, in which the contribution of urea to total urinary solute was abruptly increased.

Urea was given to patients after water deprivation and repeated injections of Pitressin 1 , and to hydropenic subjects and dogs during mannitol or sodium sulfate diuresis. Shortly after urea was given solute concentration and excretion rate increased. The $U_{\text {osm. }} / P_{\text {osm. }}$. rose or was unchanged. During osmotic diuresis, distal water reabsorption $\left(\mathrm{T}^{\mathrm{c}} \mathrm{H}_{2} \mathrm{O}\right)$ was constant over a wide range of urine flow, irrespective of antecedent diet, but increased by 20 to 35 per cent when urea was added.

It would appear that the type of urinary solute affects the capacity for water reabsorption. Possibly urea, by polymerization, by aggregation with other urinary solutes, or by partial reabsorption, is not completely effective in the determination of a limiting osmotic gradient at the site of urine concentration. Tubular secretion of urea at some more distal site cannot be excluded. In each circumstance, urea would offer less resistance to water reabsorption than is implied by its molar concentration in the urine. Thus the increased urine concentrating ability induced by dietary protein may be due solely to augmented urea excretion.

\section{Results of Clinical Application of Certain Microbiologic and Metabolic Observations in the Chemotherapy of Pulmonary Tuberculosis. W. F. RusserL, JR., S. H. Dressler, and G. Middlebrook,* Denver, Colo.}

According to one microbiologic theory it should be possible to treat effectively with some combination of two sterilizing antimicrobial agents even the most advanced cases of tuberculosis, i.e., without emergence of drugresistant mutants and with bacteriologic sterilization of all "open," active, tuberculous lesions. For achieving this result, two specific conditions were deemed essential: 1) The metabolic disposition, hence the dosage, of these agents must be considered in relation to the problem of delivering a sufficient concentration of each to all bacilli in active lesions; and 2) The microbial population excreted must be susceptible to both agents.

To test this hypothesis, 113 consecutive patients excreting populations of tubercle bacilli susceptible to both isoniazid and streptomycin were observed for at least 6 months under treatment with daily high dosages of these drugs (streptomycin for at least 90 days). Additional pharmacologic agents were administered to certain patients, but in no instance were "physical" ancillary measures employed, such as strict bed rest, pneumotherapy, or thoracic surgery, earlier than 4 months after bacteriologic conversion. Within 6 months all patients, including 46 subjects with advanced cavitary, bilateral disease, became bacteriologically negative by all criteria; and no drug-resistant mutants had emerged.

Among a control group of 104 patients consecutively admitted to hospital during the same period, who were excreting tubercle bacilli resistant to either or both drugs as a consequence of previous treatment, and who were treated with these and other antimicrobial agents in equally high or higher dosages, plus ancillary physical measures, only 21 achieved bacteriologic conversion: 5 converted coincident with surgical intervention and 12 were excreting populations susceptible to one of these two drugs. Only 4 of these 21 were excreting populations resistant to both drugs on admission to this study.

These results confirm the microbiologic theory.

Whole Blood Transaminase Levels in Anemia. MARTIN SAsS and PaUl W. Spear, Brooklyn, N. Y. (Introduced by Janet Watson).

In a study on the glutamic-oxaloacetic transaminase (G.O.T.) level of whole blood, three groups of patients were investigated. These consisted of 23 patients with no hematological abnormalities (mean hemoglobin 13.2 gm. per cent), 18 patients with mild to severe nonhemolytic anemia (mean hemoglobin $8.7 \mathrm{gm}$. per cent) and 18 patients with mild to severe hemolytic anemias, both congenital and acquired (mean hemoglobin 8.8 per cent). The mean G.O.T. level for the first two groups was 560 and 610 units per $100 \mathrm{mg}$. hemoglobin, respectively. The mean G.O.T. level for the hemolytic anemia group was 1,310 units per $100 \mathrm{mg}$. hemoglobin.

Two patients with acquired hemolytic anemia and two with megaloblastic anemia were followed during therapy. Elevated G.O.T. levels (1,750 and 1,650 units) were obtained for the former at the start of steroid therapy. Marked reticulocytosis and severe anemia were also present. Subsequent G.O.T. levels remained abnormally high (1,350 and 1,150 units) despite a drop in reticulocytosis to below 1 per cent and a rise in hemoglobin to normal or near normal values.

Normal G.O.T. values (460 and 480 units) were obtained for two patients with megaloblastic anemia prior to therapy. Ten days after the start of $B_{12}$ therapy there was a sharp elevation in G.O.T. level to 1,080 and 1,340 units together with a marked reticulocyte response. This high level of transaminase activity was maintained 
despite both the drop in reticulocytes to below 1 per cent and the increase in hemoglobin to near normal values.

A similar time relationship between reticulocyte count, hemoglobin level and transaminase level was obtained in two rabbit experiments during an episode of phenylhydrazine induced hemolytic anemia.

These preliminary results indicate that, in the limited number of patients studied, whole blood transaminase values seem to reflect a younger than normal mean red cell population due to markedly increase erythropoietic activity. Further studies are being carried out to evaluate the significance of these preliminary findings.

\section{Exchange of Ceruloplasmin Copper with Ionic Copperst and Its Relation to Wilson's Disease. I. HerRert Scheinberg * and Anatol G. Morell, New York, N. Y.}

Almost all of the plasma copper of normal subjects is tightly bound to the plasma copper-protein, ceruloplasmin. This protein is deficient or absent in patients with Wilson's disease. Several investigators have postulated that the low concentration of this protein leads to the excessive absorption of dietary copper which is apparently the fundamental metabolic error underlying this illness. However, a mechanism whereby a deficiency of ceruloplasmin would result in increased absorption of copper has not yet been proposed.

In an attempt to study this problem, experiments were performed to determine whether the copper of ceruloplasmin could be reversibly dissociated from the protein. Solutions of a human plasma protein fraction of which about 85 per cent was ceruloplasmin were mixed with radioactively labelled ionic copper both in the presence and absence of ascorbic acid. Essentially all of the ionic copper was then removed by passing the solutions through columns of ion-exchange resins. Radioactivity and the concentration of ceruloplasmin copper were measured in the effluent solutions. It was found that ceruloplasmin had exchanged about half of its copper for ionic copper in the presence of ascorbic acid, although no exchange had occurred in solutions without ascorbic acid. Since this exchange was shown not to have altered the protein with respect to its blue color, copper content, or enzymatic activity, it appears that at least half of the copper-protein bonds of ceruloplasmin are reversibly dissociable.

On the basis of these results it is possible to draw tentative conclusions about the conditions required for reversible release of ceruloplasmin copper, and to consider how such a release may bear on the relation between ceruloplasmin deficiency and the disordered copper metabolism of patients with Wilson's disease.

Congenital Defects in Bilirubin Metabolism. RuDr Schmid,* Julius Axelrod, Lydia Hammarer, and Ira M. Rosenthal, Bethesda, Md., and Chicago, IIl.

It has recently been found that bilirubin is excreted in the bile as a glucuronide. In vitro, biosynthesis of bilirubin glucuronide was obtained by incubating crystalline bilirubin with uridine diphosphate glucuronic acid and liver microsomes. The reaction product was converted to its azoderivative, which, after paper chromatographic separation, was measured spectrophotometrically and hydrolyzed for determination of the glucuronic acid.

In homozygous animals of a mutant strain of Wistar rats, the liver microsomal enzyme system catalyzing glucuronide formation was not detectable. As a result of this biochemical lesion, the rats have a congenital nonhemolytic jaundice characterized by retention of unconjugated bilirubin in the serum but without bilirubin excretion in the urine. Secretion of bile is unimpaired, but the bile is almost colorless and contains only traces of unconjugated bilirubin. In vitro and in vivo studies demonstrated that the enzymatic defect in glucuronide formation includes, in addition to bilirubin, conjugation of o-aminophenol, anthranilic acid, and menthol, but conjugation with glycine is not impaired.

A similar defect appears to exist in children with congenital non-hemolytic jaundice. In studying serum from three such patients, all the bilirubin (20 to $35 \mathrm{mg}$. per cent) was found to be of the unconjugated type, and bilirubinuria was absent. Liver histology and conventional liver function tests, including sulfobromophthalein and cholografin excretion were normal, but the bile was almost colorless and contained only traces of unconjugated bilirubin. Glucuronide formation of menthol, salicylate, and of the metabolites of hydrocortisone was markedly depressed.

These findings indicate that impaired pigment excretion in the bile with bilirubin retention in the serum may result, both in man and in animals, from defective conjugation of bilirubin. In jaundice, due to failure in glucuronide formation, the serum contains unconjugated bilirubin and bilirubinuria is absent, whereas in obstructive jaundice, serum and urine contain conjugated bilirubin.

Elevation of Peripheral Blood Ammonia Following Muscular Exercise. ARTHUR E. Schwartz, Walter LAwRence, Jr., and Kathleen E. Roberts,* New York, N. $Y$.

The hyperpnea observed during and immediately following muscular exercise has been ascribed to changes in blood $\mathrm{pH}, \mathrm{PCO}_{2}$, anoxia, and reflexes from exercising muscles or great veins. None of these alterations can account completely for the intensity of the hyperpnea. It has been suggested, therefore, that there is still an unrecognized respiratory stimulant contributing to the increase in ventilation during and following muscular exercise. The possible role of ammonia in this respect is worthy of consideration since it has been shown to stimulate respiration in both patients and dogs. It is known that the ammonia content of venous blood draining an exercised forearm muscle is elevated. The peripheral blood ammonia has not been measured following voluntary muscular exercise and it is not known whether the elevation would be sufficient to play any part in stimulating respiration. For these reasons studies were 
carried out on human subjects, in an attempt 1) to determine if there is a significant elevation of peripheral blood ammonia following voluntary exercise or following convulsions induced by electro-shock therapy and 2) to correlate the changes in blood ammonia with changes in ventilation following voluntary muscular exercise.

The results of these studies show that peripheral blood ammonia concentration increased from the normal of 1.0 microgram per cc. to values ranging between 2.1 to 3.1 micrograms per cc. in nine patients following convulsions produced by electro-shock therapy. A similar increase in blood ammonia occurred following voluntary muscular exercise. Although hyperpnea occurred while the blood ammonia was elevated, there was not a good correlation between the actual concentration of ammonia and the degree of hyperventilation following voluntary muscular exercise.

Alkalinization by Gastrodialysis as a Means of Preventing $\mathrm{CO}_{\mathbf{2}}$ Narcosis. Belding H. SCRIBNer, August G. Swanson, and James M. Burnell, Seattle, Wash. (Introduced by Robert S. Evans).

Oxygen therapy often causes coma in anoxic patients with $\mathrm{CO}_{2}$ retention; relief of anoxic stimulus to respiration decreases ventilation causing further retention of $\mathrm{CO}_{2}$. If coma results from low $\mathrm{pH}$ rather than elevated $\mathrm{pCO}_{2}$, then production of metabolic alkalosis prior to institution of oxygen therapy should protect the patient.

Alkalinization was attempted in a 67-year-old male with emphysema and severe cyanosis. Initial serum bicarbonate was $40 \mathrm{mEq}$. per L.; blood $\mathrm{pH}$ was 7.32 . Cyanosis disappeared but the patient became unconscious after 15 minutes on oxygen. He regained consciousness several hours after oxygen was withdrawn. During the next 18 hours gastrodialysis against sodium carbonate using the cellophane bag technic of Schloerb was performed. Dialysis resulted in a rise in serum bicarbonate to $64 \mathrm{mEq}$. per $\mathrm{L}$. and venous $\mathrm{pH}$ to 7.54 . Sodium balance was (-) $225 \mathrm{mEq}$. due to high urinary $\mathrm{NaHCO}_{2}$.

Oxygen again cleared cyanosis despite decreased ventilation. Venous $\mathrm{pH}$ fell to 7.40 , but there was sustained improvement of sensorium. Renal acid and ammonia excretion, 20 and $24 \mathrm{mEq}$. per 24 hours, sustained the serum bicarbonate at $63 \mathrm{mEq}$. per $\mathrm{L}$.

After 48 hours oxygen was discontinued. Nine hours later arterial oxygen saturation was 44.2 per cent; $\mathrm{pH}$ was 7.54 and bicarbonate $62 \mathrm{mEq}$. per L., as he breathed heavily at $9.6 \mathrm{~L}$. per min. On 50 per cent oxygen his breathing slowed to $6.7 \mathrm{~L}$. per min. Arterial oxygen rose to 83 per cent and $\mathrm{pH}$ fell to 7.45 . On 100 per cent oxygen breathing was $3.5 \mathrm{~L}$. per min., arterial oxygen 100 per cent, and $\mathrm{pH}$ 7.34. His sensorium remained clear.

These results suggest: 1) Loss of consciousness with oxygen therapy is related primarily to acidosis rather than elevation of $\mathrm{CO}_{2}$ tension. 2) It may be possible to give oxygen safely to such patients if they are first made alkalotic. 3) The kidneys will sustain the resultant high serum bicarbonate when oxygen therapy slows respiration and returns blood $\mathrm{pH}$ to normal.
The Nature of the Metabolic Defect in Alcaptonuria. J. E. SegGmiller, Bert N. LADu, Vincent G. ZaNNoni, and Leonard Laster, Bethesda, Md. (Introduced by George K. Hirst).

Alcaptonuria is a rare, hereditary disease in which an impaired metabolism of the aromatic amino acids, phenylalanine and tyrosine, results in the excretion of large quantities of homogentisic acid in the urine. In time, the alcaptonuric patient usually develops ochronosis, a clinical condition characterized by the deposition of melanin-like pigment in the cartilages, tendons and other connective tissues. Such deposits in the joint structures may be associated with a degenerative arthritis.

It is believed that normally tyrosine and phenylalanine are catabolized in the liver through a series of enzymatically catalyzed reactions in which homogentisic acid is an intermediate compound, and that in the alcaptonuric patient there is a block in the oxidation of homogentisic acid.

An opportunity to characterize this metabolic defect more precisely was recently afforded by the necessity to repair an hiatus hernia in such a patient. A small piece of the liver was removed during surgery and an homogenate of the specimen was assayed for the various enzymes involved in tyrosine oxidation. High activities were found for the individual enzymes catalyzing the conversion of tyrosine to homogentisic acid and for those required to catabolize maleylacetoacetic acid (the immediate product of homogentisic acid oxidation) to acetoacetic acid. The enzyme required to oxidize homogentisic acid to maleylacetoacetic acid, homogentisicase, could not be demonstrated. No evidence was found for the presence of an inhibitor of homogentisicase in the patient's liver, and the addition of ferrous ion, the only known cofactor, did not result in activity. Since homogentisicase activity in human autopsy liver from a nonalcaptonuric patient was readily detected, it seems reasonable to conclude that the metabolic block in alcaptonuria is due to a lack of homogentisicase. Thus, the hereditary defect may represent a complete inability to synthesize the enzyme or an inability to produce it in adequate quantities.

\section{Isolation Studies on Human Menopausal Gonadotrophins.} Albert Segaloff* and Sanford Strelman, New Orleans, La., and Houston, Tex.

Gonad stimulating hormone was concentrated from pooled postmenopausal urine by the Kaolin method of Albert. A two-fold concentration of the gonadotrophic hormone activity was achieved by extracting the solids with 10 per cent ammonium acetate and 59 per cent ethyl alcohol. This concentrate was processed by adsorption on DEAE cellulose and eluted from the columns with gradient elution by salts with a maximum concentration of $0.5 \mathrm{M}$ sodium chloride and $0.1 \mathrm{M} \mathrm{NaH}_{2} \mathrm{PO}_{4}$. After repeated chromatography, it has been possible to isolate two materials which give preliminary evidence of homogeneity and which contain gonadotrophin hormone activity of a high order. Both of these materials contain FSH (method of Steelman and Pohley), and LH activity 
(hypophysectomized rat ventral prostate). Although these preparations account for the major part of the $\mathrm{LH}$ activity which was recovered, it is not increased in these preparations proportionately to the increase in FSH activity. The first preparation which appears to be homogeneous by electrophoresis at two different $\mathrm{pH}$ 's and by ultracentrifugal studies shows an approximate eight-fold increase in FSH activity over the crude Kaolin material. The second fraction, for which at present we have only paper electrophoresis evidence of homogeneity, shows an approximate fifty-fold increase in FSH activity over the crude extract.

A Model for an Immunoreaction Involving Antibody, Haptene, Platelets, and Complement, in Quinidine Purpura. N. Raphaez Shulman, Bethesda, Md. (Introduced by Thomas McP. Brown).

Patients who develop thrombocytopenic purpura due to sedormid sensitivity have in their serum an antibody which fixes complement only when the specific drug (haptene), antibody, and platelets are present together. J. S. Ackroyd determined this and other characteristics of the antibody. The antibody present in quinidine purpura reacted qualitatively in the same manner. This immunoreaction was studied in detail because it may exemplify a general type of immunologic response, with relevance to other diseases of sensitization.

Analysis of quantitative relationships between components in the antibody-quinidine-platelet complex with respect to the amount of complement fixed by the complex led to development of the following model for the molecular associations involved: First, quinidine and antibody combine. Platelets provide a surface containing sites to which antibody can attach but are not part of the antigen as previously supposed. An antibody binding one, two, or three quinidine molecules can attach to platelet sites. Complement is fixed between attached antibodies only when antibodies are in nearest-neighbor position and bind two quinidine molecules. Results calculated using equations based on this model (adaptations of Langmuir's adsorption isotherm) were consistent in all respects with experimental results.

It is of particular interest that this model presents a specific mechanism for fixation of complement by an antibody reaction.

In in vivo experiments on sensitized patients, thrombocytopenia occurred at concentrations of haptene too low to cause measurable complement fixation or platelet agglutination by generally employed techniques. By varying the concentration of reactants according to principles of the above model, sensitivity of these techniques can be increased; and they may be applicable in other diseases in which this type of immunoreaction is suspected.

The Enzymatic Lesions in Galactosemia. JAMrs B. SidBURY, JR., Baltimore, Md. (Introduced by Victor A. Najjar).

Galactosemia is a familial disease characterized by intolerance to dietary lactose and galactose. Galactose-1- phosphate accumulates in the tissues of these patients when there is galactose in the diet due to the demonstrated deficiency of the enzyme "P-gal transferase." The symptoms of galactosemia presumably result from this accumulation of galactose-1-phosphate, since all symptoms abate when galactose is removed from the diet.

It has been shown that galactose-1-phosphate reacts with phosphoglucomutase at approximately $1 / 400$ the rate of glucose-1-phosphate. The mechanism of action of phosphoglucomutase has been shown to involve the transfer of phosphate from the phosphorylated enzyme to the 6 position of glucose-1-phosphate with the formation of glucose-1,6-diphosphate (the coenzyme of the reaction) and dephospho-enzyme. The glucose-1,6-diphosphate reacts with dephospho-enzyme to produce glucose-6phosphate and regenerated phospho-enzyme. In this report galactose phosphates are shown to be inhibitory to phosphoglucomutase. This inhibition is competitive in nature and explicable within the tenets of the demonstrated mechanism of action of phosphoglucomutase. Each molecule of galactose phosphate which reacts with phosphoglucomutase consumes the transferable phosphate producing galactose-1,6-diphosphate and dephospho-enzyme. The dephospho-enzyme is rephosphorylated by any glucose-1,6-diphosphate present but this is soon depleted and dephospho-enzyme accumulates because the reaction between galactose-1,6-diphosphate and dephospho-enzyme is the excessively slow step. Thus the over-all effect is the depletion of the coenzyme glucose-1,6-diphosphate and the accumulation of the dephospho form of phosphoglucomutase which is non-reactive with glucose-1-phosphate or glucose-6-phosphate.

The phosphoglucomutase appears to be essential in a number of reactions, including metabolic steps in glycolysis, polysaccharide synthesis, nucleotide synthesis. The effect of inhibiting these reactions would be far reaching and could explain the symptomatology of galactosemia.

The Role of Glucose Oxidation in the Synthesis of Cholesterol and Fatty Acids. Marvin D. Siperstein and Violet M. FAGAN, Dallas, Tex. (Introduced by Elias Strauss).

To determine how glucose oxidation influences lipid synthesis, the rate of production of fatty acids and cholesterol was measured in rat liver homogenates during stimulation of the two glycolytic pathways-EmbdenMeyerhof (EM) pathway by diphosphopyridine nucleotide (DPN) and hexosemonophosphate (HMP) pathway by triphosphopyridine nucleotide (TPN). Synthesis of fatty acids and cholesterol was measured by rates of incorporation of acetate- $\mathrm{C}^{16}$ into these compounds.

Stimulation of EM pathway by addition of DPN slightly accelerated fatty acid synthesis and had no effect upon cholesterol production. However, when glycolysis over HMP was stimulated by addition of TPN to homogenates, an 11- to 130-fold increase in fatty acid synthesis and approximately a 100-fold increase in cholesterol synthesis was noted. Simultaneous stimulation of both pathways further increased fatty acid synthesis, but 
depressed cholesterol synthesis relative to that caused by TPN alone.

Since glycolysis via either pathway yields energy, but only HMP glycolysis generates TPNH, it seemed likely that this cofactor was responsible for accelerating cholesterol and fatty acid synthesis during HMP glycolysis. This hypothesis was supported by the fact that a different TPNH generating system, isocitric dehydrogenase, was capable of substituting for HMP in enhancing lipid synthesis.

It is concluded that the hexosemonophosphate pathway may exert its major function in cellular metabolism, not simply by furnishing energy, but rather by functioning as a TPNH generating system. Since synthesis of fatty acid and cholesterol is determined in large part by availability of TPNH, the HMP pathway may provide the principal means whereby glycolysis regulates cholesterol and fatty acid metabolism.

Finally, the rate of cholesterol synthesis in the cell seems to be controlled by the relative amounts of glucose utilizing each glycolytic pathway, HMP glycolysis stimulating and EM glycolysis depressing this process.

\section{Esophageal Catheterization Studies-The Mechanism of Swallowing in Normal Subjects and Patients with Dysphagia with Particular Reference to the Esophago- Gastric Sphincter (Vestibule). Marvin H. SleIsenger and James H. Pert, New York, N. Y. (Introduced by Thomas P. Almy).}

By means of polyethylene catheters pressures have been recorded simultaneously from three sites in the esophagus by means of electromanometers and a Sanborn recorder. A pump maintained a flow of $3 \mathrm{cc}$. water per hour through the catheters. Catheter positions were checked by fluoroscopy and documented with spot films. Attention was focused upon motility during deglutition. Twenty-two studies were performed on five normal subjects, six patients with cardiospasm and six individuals with intermittent "functional" dysphagia.

There are distinctive swallowing patterns for the upper $8 / 9$ of the esophagus (body) and lower $1 / 9$ (vestibule). Five components of the body wave are identified; the largest represents peristalsis which usually attains pressures of 15 to $20 \mathrm{~cm}$. water and lasts 8 to 10 seconds. This wave travels at a rate of 2.0 to $3.0 \mathrm{~cm}$. per second and stops at the vestibule.

The deglutition complex of the vestibule, the terminal 2 to $5 \mathrm{~cm}$. of esophagus, is characterized by brief relaxation followed by contraction with a rise in pressure to $\mathbf{1 5}$ to $20 \mathrm{~cm}$. water which lasts for 20 seconds. Strikingly, the pressure will suddenly drop, in a second or less, to baseline. This pressure rise begins as peristalsis is progressing down the body. The failure of peristalsis to enter the vestibule, the height of pressure reached and its sudden relaxation during deglutition are consistent with the action of a sphincter.

In cardiospasm the narrowed distal segment corresponds to the vestibular sphincter. It has a high resting pres- sure, fails to relax in a coordinated manner during swallowing and generates inordinately high pressures, occasionally reaching $100 \mathrm{~cm}$. water for as long as $15 \mathrm{~min}$ utes. Other abnormalities of this condition include : failure of progression of peristalsis in the body and the responses of both body and vestibule to autonomic drugs.

The esophagus in patients with "functional" dysphagia demonstrates progress of peristalsis in the body and also reacts normally to drugs but has statistically higher vestibular pressures for longer periods of time than is normal.

The Estimation of Non-thyroxine Iodine Released by the Thyroid Gland in Hyperthyroidism. D. WARD SLINGERLAND and Belton A. Burrows,* Boston, Mass.

Although small amounts of organic iodide compounds other than thyroxine have been identified in sera of hyperthyroid patients, the major component of circulating organic iodine is thyroxine. The present study indicates that the hyperthyroid gland may release non-thyroxine iodine, either as iodide or some rapidly metabolized organic iodine compound, in amounts approaching the secretion of thyroxine iodine.

Following a six to eighteen-day period of equilibration with a tracer dose of radioiodine, the effect of antithyroid compounds and iodides on thyroidal radioiodine, serum protein-bound radioiodine and chemical iodine, and urinary radioiodine excretion was determined. With Tapazole ${ }^{\circledR}$, urinary radioiodine excretion was increased in normal subjects several times more than would result from inhibition of thyroid uptake of radioiodide recirculated from body tissues, but in hyperthyroid subjects the increase in urinary radioiodine could come entirely from peripheral hormonal degradation. As one possible explanation for this finding, in normal subjects there may be interference with reutilization of radioiodine released by intrathyroidal deiodinating mechanisms, but in hyperthyroidism intrathyroidal recycling of iodine may not occur.

As antithyroid compounds were continued, a decline in urinary radioiodine excretion relative to serum proteinbound radioiodine levels was noted. Occasionally, administration of iodides in addition to Tapazole resulted in immediate reduction in urinary radioiodine excretion amounting to more than one-half previous values. Concomitant slowing in the slope of thyroidal radioiodine, with a constant slope in serum protein-bound radioiodine and chemical iodine values, suggested an inhibition of thyroid release of radioiodine in some form other than thyroxine.

Secretion of significant amounts of non-thyroxine iodine, resulting from incomplete intrathyroidal deiodination of organic iodine compounds, incomplete reutilization of iodide released by such deiodinating mechanisms, or incomplete iodination of organic iodine compounds, may be of primary importance in the pathogenesis of hyperthyroidism and the response to antithyroid compounds and iodide. 
A Mechanism for Hypoalbuminemia in Patients with Ulcerative Colitis and Regional Enteritis. JESSE L. Steinfeid, John D. Davidson, and Robert S. Gordon, JR., Bethesda, Md. (Introduced by A. I. Mendeloff).

The hypoalbuminemia frequently found in patients with ulcerative colitis and regional enteritis has been presumed to be due largely to poor absorption of essential nutrients. It has been suggested that loss of protein into the lumen of the bowel might also contribute to the hypoalbuminemia in these patients.

By immunochemical methods both albumin and gamma globulin have been demonstrated in the succus entericus of a patient with regional enteritis whereas neither was detectable in specimens from normal individuals. Electrophoretic analyses revealed both hypoalbuminemia and hypogammaglobulinemia in two patients with regional enteritis.

A single injection of $50 \mathrm{mgm}$. of human serum albumin labeled with 100 microcuries of $\mathrm{I}^{1 \mathrm{kn}}$ has been administered intravenously to patients with regional enteritis or ulcerative colitis and to patients without gastrointestinal disease; blood samples, stools and urine were collected for periods up to four weeks. Whereas in patients without gastrointestinal disease, 0.1 to 1.0 per cent of the total daily $\mathrm{I}^{120}$ excretion was found in the feces, patients with diarrhea due to regional enteritis or ulcerative colitis excreted 10 to 50 per cent of the radioactivity in the stools. Data on the stool:urine partition of radioactivity following the intravenous injection of iodide ${ }^{181}$ and $\mathrm{I}^{181}-1 \mathrm{la}-$ beled albumin in individuals without gastrointestinal disease before and after the induction of diarrhea will be presented.

Administration of tracer quantities of $\mathrm{I}^{\mathrm{In}}$-labeled human serum albumin to patients with regional enteritis and ulcerative colitis has revealed their total exchangeable albumin to be sub-normal. However, albumin turnover (grams per kilogram of body weight per day) was greater than normal; the fraction of the total albumin pool renewed each day was approximately twice normal. Since albumin turnover is actually increased in these patients it is extremely unlikely that poor absorption of protein precursors is the principal cause of the hypoalbuminemia.

The Rate of Degradation of Thyroxine in Patients with Thyroid Disease after Therapy. KenNeth StERLING,* Syracuse, N. Y.

The disappearance curve of plasma radioactivity after intravenous injection of $\mathrm{I}^{100}$ labeled 1-thyroxine was employed to determine the rate of hormone degradation or removal. Studies were carried out before and after therapy in patients with thyroid disease.

Thyroxine degradation was slower than normal in untreated myxedema, and faster than normal in thyrotoxicosis. Follow-up studies were carried out after appropriate therapy and attainment of the euthyroid state. Euthyroidism was evaluated by clinical appearance, basal metabolic rate, and plasma protein bound iodine concentra- tion. The initially abnormal disappearance curves had usually reverted to normal or nearly so when patients were restudied two months to several years after treatment.

In five myxedematous patients on prolonged replacement therapy, the degradation rate had returned to the normal range or slightly below. In contrast, no alteration in the disappearance curve occurred in subjects followed for one to three weeks after rapid treatment with triiodothyronine.

After treatment of thyrotoxicosis with radioiodine, surgery, or antithyroid drugs, the degradation rate became normal or approximately normal in seventeen subjects. In one instance the rate remained markedly accelerated despite clinical euthyroidism. In two cases of post-radioiodine hypothyroidism, the rate was as slow as that seen in spontaneously occurring myxedema.

The results suggest that the rate of removal of circulating thyroxine is a significant parameter of thyroid hormone metabolism.

Observations on Deoxyribonucleic Acid Synthesis by Human Bone Marrow Cells in Vitro. E. Donnall Tномаs, Cooperstown, N. Y. (Introduced by James Bordley, III).

By available criteria of cellular viability, bone marrow in vitro appears to survive at best for only a few days. The evaluation of minor changes in cell count during this short period is difficult and represents a balance between cell production and cell destruction. Deoxyribonucleic acid (DNA) has been shown to be metabolically stable, and measurement of the rate of its formation is considered a direct function of cell proliferation. Isotopic formate is incorporated into the methyl group of thymine by bone marrow cells. Since thymine occurs only in DNA, it appears that DNA synthesis can be followed by the direct isolation of thymine without the necessity of separating DNA from ribonucleic acid. This paper describes studies of DNA synthesis by normal human bone marrow in vitro by measuring the rate of incorporation of $\mathrm{C}^{14}$-formate into thymine.

It was found that DNA synthesis continues at an approximately constant rate for several hours. The optimal formate concentration was found to be 0.5 micromole per ml. and optimal $\mathrm{pH}$ approximately 8 . The presence of serum is essential for optimal DNA synthesis, and it cannot be replaced by currently available synthetic media. The effect of X-irradiation on normal human bone marrow was studied. Results indicate that as little as $200 \mathrm{r}$ of X-ray interferes with DNA synthesis. However, heme synthesis was unaffected by doses less than $1,000 \mathrm{r}$. Data concerning the time factor in the effect of X-ray on human bone marrow will be illustrated.

The method appears to be reproducible and to make it possible to follow rates of DNA synthesis over very short periods of time and with small amounts of marrow. It should prove useful in studying many of the problems concerned with the proliferation and viability of bone marrow cells. 
Studies on a Physiologically Important Enzymatic Re-

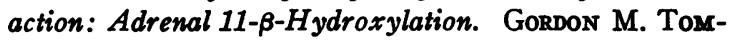
kINS, Bethesda, Md. (Introduced by Joseph $\mathrm{E}$. Smadel).

The introduction of an 11-hydroxyl group into the steroid nucleus is an essential step in the biosynthesis of the physiologically active adrenal steroids cortisol (compound $F$ ), corticosterone (compound $B$ ), and aldosterone. Information about this reaction is, therefore, of the utmost importance.

By the use of partially purified cell-free extracts of the adrenal and other organs, we have demonstrated that at least two distinct enzymes are involved in this process. One of these is present only in adrenal mitochondria. The other, however, is much more widely distributed and has been detected not only in the adrenal, but also in the lung, spleen, liver, and serum.

We have observed that amphenone $B$, used clinically by Hertz and his associates to depress adrenal cortical function, is a potent inhibitor of the 11-hydroxylating enzyme system. Diphenyl propyl acetic acid (S.K.F. no. 2314), an inhibitor of drug metabolism, and chemically somewhat similar to amphenone, has also been found to block adrenal 11-hydroxylation in vitro. Furthermore, it has been found that the activity of the hydroxylating enzyme system is inversely proportional to the ionic concentration of the medium even within physiological limits. Although it is not possible at this time to assess whether or not this inhibition by salts is of any homeostatic significance, it is interesting to note that the direction of the effect (that is, inhibition of hydroxylation by high ionic strength) would serve to maintain a constant intracellular ionic environment by inhibiting the synthesis of salt-retaining hormones.

It would appear that a detailed examination of the enzymatic hydroxylating system might provide insight into chemical mechanisms which are of physiological and clinical importance.

Perfusion Studies of the Human Placenta: Hormonal Effects on Citric Acid Metabolism. PHILIP Troen and Edwin E. Gordon, Boston, Mass. (Introduced by Samuel L. Gargill).

The factors controlling placental metabolism and the role of human chorionic gonadotropin (HCG) in this process have not been established. In initial studies of this problem the metabolic activity of the placenta has been determined utilizing perfusion techniques. Villee and associates have shown that the utilization of citric acid by placental homogenates is enhanced by estradiol stimulation of a DPN-dependent isocitric dehydrogenase. Accordingly, freshly obtained human placentas were perfused for several hours with a modified Tyrode's solution containing citric acid. Hourly measurements were made of citric acid utilization and $\alpha$-keto acid production. The effect of hormones added to the perfusing fluid was studied in four groups of three placentas each: 1) control,
2) estradiol-17ק, 3) HCG, 4) estradiol and HCG combined.

The control group had an average total utilization of $854 \mathrm{mgm}$. citrate per placenta and an average total accumulation of $23.4 \mathrm{mgm}$. of $\alpha$-keto acid (expressed as $\alpha$-keto-glutaric acid) per gland. Addition of either estradiol or HCG separately to the perfusate did not result in a significant difference from these total values. However, when estradiol and HCG were added simultaneously there was a significant increase in average total citrate utilization to $1615 \mathrm{mgm}$. per gland. The hour-to-hour values also revealed significant increasing utilization of citrate by the placentas in this group. In addition, the total accumulation of $\alpha$-keto acid dropped to an average total of $13 \mathrm{mgm}$. per gland in this group.

These data demonstrate metabolic activity persisting in human placentas for at least eight hours of perfusion. An effect of HCG on such metabolic activity has been shown for the first time. This methodologic approach permits study of the factors controlling placental metabolism and may provide clarification of the endocrine functions of the human placenta.

The Fatty Acids of Normal Plasma and of Arteriosclerotic Plaques. NAIP TUNA, LoIs Reckers, and Ivan D. Frantz, JR.,* Minneapolis, Minn.

The literature contains suggestions that the saturated fatty acids, by virtue of their relative insolubility, may deposit preferentially in the arterial wall, thus playing a special role in the development of atherosclerosis. In an attempt to settle this question, we have analyzed the fatty acids of total lipides and of cholesterol esters from pooled plasma of normal persons, and from atheromatous plaques obtained from autopsy material. The total lipides were extracted with chloroform-methanol. The cholesterol esters were separated from other lipides by silicic acid chromatography. The fatty acids were first partially separated by counter-current distribution. The peaks were then analyzed by iodine value, alkali isomerization, paper chromatography before and after hydrogenation, and vapor phase chromatography.

The most important conclusions to be drawn from these analyses are as follows: (1) The fatty acid spectrum of the total lipides of plaques is very similar to that of serum. Both contain about 30 per cent of oleic acid, with slightly smaller amounts ( 20 to 25 per cent) of linoleic and palmitic acids. In addition to these constituents, the mixture contains 4 to 7 per cent each of stearic, arachidonic, and $C_{10}$ unsaturated acids, as well as traces of other components. (2) The fatty acids of cholesterol esters include about 40 per cent of linoleic acid. Otherwise they do not differ greatly from the fatty acids of total lipides. (3) The cholesterol esters of serum and plaques are quite similar.

Although it is still possible that "essential" fatty acids are important in the prevention of atherosclerosis, the present findings indicate that any such effect is not due to their inability to participate in plaque formation. 
Zinc Metabolism in Post-Alcoholic Cirrhosis. BERT L. Vallee,* Warren E. C. Wacker, and Jeremias H. R. KaEgI, Boston, Mass.

Iron in hemochromatosis and copper in Wilson's disease are implicated in the pathogenesis of these diseases, both of which ultimately lead to cirrhosis. Zinc, another transition-element, participates in the dehydrogenation of ethanol. This led to studies of zinc in post-alcoholic cirrhosis. In normal individuals $\sim 500 \mu \mathrm{g}$. per 24 hours of directly reacting zinc is excreted. Elimination of zinc in the urine apparently varies with the functional capacity of the post-alcoholic liver. "Zincuria" from $\mathbf{7 5 0}$ to 2,500 $\mu \mathrm{g}$. per 24 hours has been observed in individuals with cirrhosis and hepatomegaly; apparently the first authenticated instance of primary "zincuria" without albuminuria. One terminal, jaundiced individual, however, with a small liver, ascites, serum-zinc $49 \mu \mathrm{g}$. per cent, and BSP 61 per cent, excreted $\sim 50 \mu \mathrm{g}$. of zinc in 24 hours. Oral zinc induced transient changes in zinc excretion, and after ten days the BSP was 53 per cent. Another patient with marked hepatomegaly presented a different pattern: Serum-zinc was $77 \mu$ g. per cent, urinary zinc excretion was about $800 \mu \mathrm{g}$. per 24 hours and the BSP was 32 per cent during the control period. Oral zinc abruptly decreased urinary zinc concentration to $\sim 400 \mu \mathrm{g}$. per 24 hours. This level was maintained below the mean of the normal series throughout the experimental period. Serum-zinc rose to within normal limits. BSP was 10 per cent after ten days, remaining at this level.

The participation of zinc in the dehydrogenation of ethanol, in the metabolism of post-alcoholic cirrhosis and the tendency of physiological quantities of zinc to restore normal biochemical patterns in the individuals studied emphasizes the unsuspected participation of this transition-element in another pathologic process, characterized by severe cicatrization in its terminal stages.

Flame Spectrometry, Magnesium and the Uremic State. Warren E. C. Wacker, Boston, Mass. (Introduced by Gustave Dammin).

Somnolence and coma are frequent manifestations of the uremic state. The biochemical mechanisms leading to these clinical changes are obscure. The known pharmacological effect of magnesium bears a resemblance to certain features of this syndrome which is characterized by electrolyte disturbances.

The development of flame photometry has facilitated the study of sodium and potassium, but the chemical methods for magnesium have remained cumbersome. The physiology of magnesium and its clinical implications is not understood as well as that of the alkali metals, but the function of magnesium in enzymology has been studied intensively.

The validation of a flame-spectrometric method for magnesium has permitted the examination of this element in renal failure. The procedure has a repeatability of 1.9 per cent with excellent recovery. The normal serum magnesium follows a Gaussian distribution with a mean of $2.05 \pm 0.18 \mathrm{mEq}$. per $\mathrm{L}$.

In several patients serum magnesium concentrations varying from 3.5 to $5.5 \mathrm{mEq}$. per $\mathrm{L}$. have been observed in the presence of clinical and chemical changes characteristic of renal insufficiency. While anuria was a common feature, hypermagnesemia also occurred in the presence of a normal volume of urine. Hemodialysis when performed without magnesium in the dialyzing fluid significantly lowered the serum magnesium concentration, in accord with evidence that a fraction of this element is protein-bound. Electrocardiographic changes reported to be typical of hypermagnesemia were detected and found reversible with dialysis.

These studies indicate distinct abnormalities in magnesium metabolism of patients with some types of renal failure. The recognition of this change has important implications in the diagnosis and management of renal failure, particularly of the acute, reversible type where it may constitute a criterion for hemodialysis.

\section{Urea Breakdown in Normal Man. MACkenzIE WALSER} and Leonard J. Bodenlos, Bethesda, Md. (Introduced by Gilbert H. Mudge).

In normal subjects, the rate of urea breakdown, heretofore assumed to be negligibly small, has been determined by injecting intravenously trace quantities of urea labeled with either $\mathrm{C}^{16}$ or $\mathrm{N}^{15}$. Between the second and eighth hours after injection, blood urea isotope concentration declined exponentially in five experiments with $\mathrm{N}^{16}$-urea and in six out of seven experiments with $\mathrm{C}^{16}$-urea, indicating that appreciable reincorporation of labeled atoms into newly formed urea during this period occurred in only one instance. Therefore, it was possible to calculate the rate of urea production as the product of the pool size and the turnover rate, after making allowance for excess isotope excreted during the first two hours. Urea production exceeded the simultaneous rate of urea excretion by 15 per cent to 55 per cent (mean 23 per cent). In one week, 79.5 per cent of a dose of $25 \mathrm{mg}$. excess $\mathrm{N}^{1}$ (given as urea) was excreted in the urine as urea, 0.4 per cent as ammonia, and 9.7 per cent as undetermined nitrogen. Urinary creatinine and uric acid were not enriched. Cumulative urinary recovery of $\mathrm{C}^{\mathbf{1}}$-urea averaged 70 per cent within 46 hours (four subjects) and 81 per cent within 72 hours (three subjects). Reincorporation of label into urea occurred with $\mathrm{C}^{14}$ as well as with $\mathrm{N}^{15}$ as indicated by the fact that 1 per cent of the dose of $\mathrm{C}^{16}$ was recovered as urea between 72 and 96 hours after injection.

Oral chlortetracycline, oxytetracycline, and succinylsulfathiazole usually reduced but did not eliminate breakdown. Oral Neomycin preparation for 48 hours resulted in urinary recovery of $96 \pm 2$ per cent (s.e.) of $C^{14}$-urea as urea in six subjects in 72 hours.

Thus urea breakdown by gastrointestinal bacteria constitutes a significant pathway of nitrogen catabolism, resulting in the potential release of about 2 grams a day of ammonia into the portal circulation. 
Identification of a New Group A Streptococcal Desoxyribonuclease: The Antibody Response in Scarlet Fever and Acute Rheumatic Fever. LewIS W. WannaMAKER, New York, N. Y. (Introduced by Maclyn McCarty).

Strain variation among Group A streptococci has been explored by comparing electrophoretic patterns of concentrates of culture supernates. These studies revealed the presence of two distinct streptococcal enzymes, both of which depolymerize desoxyribonucleic acid. The two desoxyribonucleases migrate differently on starch block electrophoresis and are distinguishable serologically by differential antibody neutralization. They are designated here as DNASE I and DNASE II.

DNASE I resembles that previously described by McCarty and can be identified in preparations from the strain used in his studies. DNASE II appears to be more generally distributed among strains of Group A streptococci. Moreover, antibody studies suggest that DNASE II is a more frequently encountered streptococcal antigen than DNASE I. None of the sera from 12 normal adults had detectable antibody to DNASE I, whereas all showed some antibody to DNASE II, usually in titers between $1: 20$ and 1:80. Thus, antibody to DNASE II resembles antistreptolysin $O$ in its almost universal distribution in low titers among normal adults.

Acute and convalescent sera from 21 patients with scarlet fever were examined. Fourteen showed a rise in antibody titer to one or both of the enzymes. Six showed a rise to DNASE I, whereas 13 showed a rise to DNASE II.

In patients with acute rheumatic fever, antibody to DNASE II was demonstrated more frequently and at higher levels than antibody to DNASE I. No antibody to DNASE I was detected in 12 of 38 such patients, and only 9 showed titers of 1:80 or greater. In contrast, 34 of the 38 patients showed titers of $1: 320$ or greater to DNASE II. These data suggest that, like antistreptolysin $\mathrm{O}$, antibody to this new desoxyribonuclease (DNASE II) may be a useful index of recent streptococcal infection among patients in whom the diagnosis of acute rheumatic fever is being considered.

\section{Depression of the Total Body Respiratory Quotient in Human Malignant Disease. Donald M. Watkin, Bethesda, Md. (Introduced by Charles G. Zubrod).}

Total respiratory quotients (R.Q.'s) were measured to estimate the proportion of fat in the nutrient mixture metabolized by subjects with and without malignant disease. High fat values in subjects with progressive neoplasia had previously been computed from balance study data using Lavietes' formula. R.Q. measurements were made 15 hours post prandial at intervals of several days in each subject. Room air was inspired. Expired air was collected for 36-minute periods in Douglas bags or metered and aliquoted in a Franz-Müller gasometer. Samples were analyzed for oxygen and carbon dioxide in Beckman and Cambridge instruments, respectively. Un- der these conditions the R.Q.'s of healthy adults averaged 0.786 ; of subjects chronically ill with non-malignant disease, 0.784 ; of subjects with clinically inactive malignancies, following surgery, radiation or endocrine manipulations, 0.801 ; but of adequately nourished subjects with progressive malignant disease, 0.693 . In subjects initially demonstrating low R.Q.'s and rapidly progressing neoplasms, hypophysectomy with replacement corticoid therapy, corticoid therapy alone and large doses of radiation to extensive body areas produced temporary elevations in R.Q. and temporary objective cessation of tumor growth. Upon relapse the R.Q.'s returned to control values. The data suggest that subjects with advanced neoplasms metabolize more fat than normal adults, subjects with nonmalignant chronic diseases and subjects with arrested malignancies. They are compatible with interpretations of balance data previously derived using Lavietes' formula. Reduction of the total body basal R.Q. implies the existence of a metabolic process associated with but not necessarily dependent upon the anatomical neoplasm. This process may be modified by hypophysectomy, corticosteroids and extensive body radiation.

\section{Effect of a Carbonic Anhydrase Inhibitor, Acetazoleamide}

(Diamox (\$), on Cerebral "Ammonium" Metabolism of

Patients With and Without Hepatic Cirrhosis. LesLIE

T. Webster, Jr., and Jerome Kleinerman, Cleveland,

O. (Introduced by George J. Gabuzda).

Diamox may induce impending hepatic coma (mental confusion and a flapping tremor) associated with increased antecubital venous blood "ammonium" concentrations in susceptible patients with hepatic cirrhosis. Accordingly, ammonium metabolism of the brain was evaluated twice before and twice within 60 minutes after injecting 500 $\mathrm{mg}$. of Diamox intravenously into 8 patients with and 8 without hepatic cirrhosis. Arterial and superior jugular bulb venous blood samples were analyzed for "ammonium" (Conway), glucose (Somogyi) and oxygen (Van Slyke). Cerebral blood flows were determined before and after Diamox in 6 patients of each group by the nitrous oxide technique (Kety).

In patients without liver disease, changes in arterial "ammonium," glucose, and oxygen were not significant after Diamox 1 . Cerebral blood flow increased significantly $(p=.05)$ but cerebral uptakes of "ammonium," glucose, and oxygen were not consistently affected.

In contrast, patients with cirrhosis exhibited significantly increased arterial and jugular blood "ammonium" concentrations after Diamox $\$ 24$ per cent above the control, $\mathrm{p}<.01)$. A significant cerebral uptake (mean $=6.2$ micrograms per $100 \mathrm{gm}$. tissue per minute; $\mathrm{p}<0.05$ ) of "ammonium" occurred also although there was neither a significant uptake nor release during the control period. Neither arterial glucose concentration, oxygen content, nor cerebral uptakes of glucose or oxygen were changed, but the cerebral flow again increased $(p=.05)$ after Diamox ${ }^{\circledR}$.

Increased blood "ammonium" concentrations noted after Diamox administration to patients with cirrhosis are 
probably of endogenous origin because practically none of the nitrogen contained in this drug is released. The cerebral "ammonium" uptake indicates that "ammonium" liberation from the brain does not account for the increased arterial blood "ammonium" concentrations observed. A cerebral "ammonium" uptake is associated with impending hepatic coma induced by a nitrogen load. Whether or not the cerebral "ammonium" uptake following Diamox administration is related to the neurological deterioration induced by this drug requires further investigation.

Inhibition of the Bactericidal Activity of the Properdin System with Specific Bacterial Antibodies. RALPH J. WeDGwood, Cleveland, O. (Introduced by William M. Wallace).

The nonspecific bactericidal activity of normal human serum against Gram-negative bacteria requires properdin and has been shown to be distinct from immune mechanisms requiring antibody. This communication shows that the bactericidal activity of properdin can be specifically inhibited by antibodies to the bacterium.

Serum, in which bactericidal activity has been markedly diminished by almost complete properdin depletion, may contain antibodies to the somatic antigen of $S h$. dysenteriae. Removal of such antibodies by adsorption with the antigen restores bactericidal activity against $S h$. dysenteriae, but does not affect bactericidal properties against a heterologous organism-E. coli. Similarly, the addition of specific somatic antibodies to intact serum decreases bactericidal activity to the homologous but not to the heterologous organism. Within limits, a direct stoichiometric relationship exists between the amount of antibody, which inhibits, and the amount of properdin, which is needed, to produce bacteriolysis. This relationship is distinct from pro-zone phenomena.

It is generally considered that specific antibodies to Gram-negative bacteria may be bactericidal. The findings presented here indicate that specific antibody to the somatic antigen of $S h$. dysenteriae, rather than being bactericidal, may actually inhibit the normal nonspecific bactericidal activity of human serum. This inhibition of the bactericidal activity of the properdin system by antibody seems to be immunologically specific and probably represents competition between properdin and antibody for similar sites on the bacterium. These studies offer a new approach for determining this site of interaction of the properdin system with the bacterium itself.

The Effect of Total Adrenalectomy in a Patient with Chronic Massive Ascites Caused by Post-Necrotic Cirrhosis. EMILE E. Werk, JR., Jerome GIUSEFFI, JR., Paul U. Larson, David W. Elliott, and Yoichi Orkawa, Cincinnati, O. (Introduced by Leon Schiff).

Bilateral adrenalectomy was performed June, 1956, in a 28-year-old man to alleviate "maximum" ascites which had existed 14 months as the predominant manifestation of post-necrotic cirrhosis.

Two weeks post-operatively while receiving liberal dietary sodium and cortisone acetate (E) $37.5 \mathrm{mg}$. daily, the patient gained weight rapidly. Urinary output averaged $700 \mathrm{ml}$. per day and urinary sodium was $8 \mathrm{mEq}$. per day. Daily $\mathrm{E}$ was reduced to 25 and $12.5 \mathrm{mg}$. and sodium intake decreased. Weight remained constant. Urinary sodium ranged from 20 to $100 \mathrm{mEq}$. per day. On $8 \mathrm{mg}$. E per day weight diminished, urinary volume and sodium increased but anorexia and depression developed.

In August, a cellulitis and marked adrenal insufficiency intervened and produced a profuse diuresis which high cortisone dosage plus antibiotic administration reversed. A sustained sodium and water diuresis then ensued over the next three months. Weight decreased $27 \mathrm{Kg}$. and no ascites or edema was perceptible. Appetite and strength improved. E dosage varied from 18.75 to 50 mg. daily. At intervals prednisone was added. During the greater part of this period estimated sodium intake was approximately $100 \mathrm{mEq}$. per day, fluid intake 1,700 $\mathrm{ml}$. per day and urinary volume and sodium averaged $1,200 \mathrm{ml}$. and $120 \mathrm{mEq}$. per day, respectively. Ascites reaccumulated for a brief period in December when patient was allowed a free sodium and water intake and received hydrocortisone $25 \mathrm{mg}$. daily.

Urinary aldosterone levels (performed by Dr. Edgar Gordon) were $70 \mathrm{mcg}$. per day pre-operatively and zero one month post-operatively.

Thus adrenalectomy permitted loss of ascites and improvement with the patient maintained on moderate sodium intake and replacement cortisone. However, a defect in water and salt retention remained which was prominent two months post-operatively and lessened following an acute infection but persisted after ascites had completely disappeared.

\section{$A$ Distinction Between the Anticoagulant and Antithrom- botic Effects of DicumarolB. Stanford Wessler,* Jonathan D. Ballon, and Jay H. Katz, Boston, Mass.}

The cardinal problem in Dicumarol therapy is the determination of the amount of drug necessary to inhibit thrombosis without precipitating hemorrhage. The therapeutic dose, however, is gauged by laboratory tests which reflect specifically a coagulation defect: a parallel antithrombotic effect has been assumed. In dogs the systemic infusion of serum produces a temporary hypercoagulable state during which massive thrombosis is routinely induced in areas of retarded blood flow. This technic permits independent measurement of coagulation defects and thrombus formation. In short term experiments, serum fractions were obtained from 13 donor dogs in which the one-stage overall plasma prothrombic activity had been reduced by Dicumarol to values ranging from 25 to 6 per cent of the control values. These serum fractions were then infused into 13 additional animals in which the prothrombic activity had been comparably depressed by Dicumaroli. The coagulation defect in both the donor and recipient animals was significant; yet, thrombosis was observed in each recipient animal. Moreover, the 
partial inhibition of thrombosis found in 3 of the 13 animals could not be correlated with the one-stage overall prothrombic activity, prothrombin or convertin activities of either donor or recipient dogs. A dichotomy was thus demonstrated between the coagulation defect and the antithrombotic effect produced by Dicumarol $\oplus$. Moreover, the one-stage overall prothrombic activity, prothrombin and convertin activities did not accurately reflect this antithrombotic activity of the drug. These results provide an experimental counterpart to clinical experience in which thrombosis has occurred in Dicumarol 18 -treated patients exhibiting a marked interference with the coagulation mechanism. These findings also reinforce the need for a more appropriate measure of the antithrombotic activity of DicumaroliB than is currently available.

Water Diuresis and Antidiuretic Mechanisms in Diabetes Insipidus and Hepatic Cirrhosis. ABRAHAM G. WhITE, New York, N. Y. (Introduced by Milton Mendlowitz).

The occurrence in diabetes insipidus of: (1) subnormal diuresis after hydration, (2) edema, and (3) ascites (produced experimentally), points to an important physiological role of an extra-neurohypophysial antidiuretic mechanism in this syndrome. That an extra-neurohypophysial antidiuretic mechanism may play a major role in the water retention of hepatic cirrhosis has been suggested by studies of water diuresis, Pitressin antidiuresis, alcohol diuresis and serum antidiuretic activity in this disease.

To determine whether or not the aforementioned extraneurohypophysial antidiuretic mechanisms are similar in these two disease states, studies were made of water diuresis following intravenous hydration with 5 per cent glucose in water $(10 \mathrm{ml}$. per min.) in three patients with diabetes insipidus and four with hepatic cirrhosis. In three additional patients with diabetes insipidus data were obtained on water diuresis following oral hydration with $1,500 \mathrm{ml}$. water. Fourteen normal subjects served as controls, seven for intravenous hydration, and seven, oral hydration.

Following intravenous hydration, the four patients with cirrhosis reached a peak diuresis in $94.5 \pm 27.5 \mathrm{~min}$. with a mean peak flow of $11.9 \mathrm{ml}$. per min.; the three patients with diabetes insipidus did not attain a sharp peak of diuresis, and had a mean maximal urinary flow of $7.6 \mathrm{ml}$. per min. Control subjects attained a peak in $85.1 \pm 32.3$ min., with a mean peak flow of $12.0 \mathrm{ml}$. per min.

After the ingestion of $1,500 \mathrm{ml}$. of water within 25 min., control subjects reached a mean peak diuresis of $15.2 \mathrm{ml}$. per min., while patients with diabetes insipidus attained a mean maximal flow of $6.3 \mathrm{ml}$. per min.

The subnormal diuretic responses of patients with diabetes insipidus following both oral and intravenous hydration are interpreted as confirming the presence of extra-neurohypophysial antidiuretic mechanisms. The differences in the diuretic responses between patients with diabetes insipidus and those with cirrhosis suggest differences in the antidiuretic mechanisms operative in these two disease states.
Radioactivity of Human and Cattle Thyroids Associated with Fallout. Margaret R. White and Hardin B. Jones, Berkeley, Calif. (Introduced by John H. Lawrence).

The level of radioactive iodine in human thyroids obtained at autopsy has been followed since January, 1956, and that in thyroids of cattle slaughtered in the San Francisco Bay area since February, 1955.

No individual human thyroid of the 155 studied during this period contained enough $I^{\text {1an }}$ to be detected even by sensitive counting techniques. It was possible, however, to obtain an outside limit of the $I^{181}$ concentration in human thyroids and to estimate the maximum possible level. All the human thyroids corresponding to the period of constant and high $I^{181}$ level in range cattle thyroids (June to October, 1956) were recounted after decaying through a minimum of four $\mathrm{I}^{\text {1an }}$ half-lives. It was estimated that the average activity attributable to $\mathrm{I}^{\mathrm{Im}}$ in the human thyroids was $0.0006 \mathrm{~m} \mu \mathrm{c}$. per $\mathrm{gm}$. $(1 / 1,000$ th of the average level observed in range cattle thyroids), that there was only one chance in 1,000 that human thyroids could have received a dose exceeding 0.001 rep, and that the probable value was 0.00016 rep.

Three periods of sharply elevated radioactivity were noted in range-fed cattle thyroids. (1) The Nevada series in the spring of 1955 produced a maximum radioactivity to an individual thyroid of $6.4 \mathrm{~m} \mu \mathrm{c}$. of $\mathrm{I}^{121}$ per gram of wet tissue and delivered a total dose to rangefed cattle thyroids for the period of elevated activity of 1.0 rep. (2) The Russian test in March of 1956 produced a maximum radioactivity of $1 \mathrm{~m} \mu \mathrm{c}$. per $\mathrm{gm}$. and a total dose of 0.2 rep. (3) The Bikini tests in the spring and summer of 1956 produced a maximum radioactivity of $2.67 \mathrm{~m} \mu \mathrm{c}$. per $\mathrm{gm}$. and a total dose of 1.3 rep. The thyroids of feed-lot-fed cattle showed much lower values (generally being near background), implicating the range-plant food as the only probable source of radioactivity.

Effect of Thiamin Deficiency on Human Erythrocyte Metabolism. Stanley J. Wolfe and MYron Brin, Boston, Mass. (Introduced by Charles S. Davidson).

Augmented oxygen consumption of mammalian erythrocytes by methylene blue has been demonstrated to result from hexose monophosphate shunt activation. As this pathway cycles and after the first carbon of the hexose molecule is oxidized to $\mathrm{CO}_{3}$, the second and then the third carbons successively assume the carbon-1 position and are subsequently recoverable as $\mathrm{CO}_{2}$. A fixed ratio exists normally between carbon-1 and carbon-2 recovery. By labelling carbons 1 and 2 of glucose with $\mathrm{C}^{14}$, blocking of the cycle was demonstrated in thiamindeficient rat erythrocytes, as evidenced by greatly reduced carbon-2 recovery and increased pentose accumulation.

Erythrocytes from patients with thiamin deficiency, Wernicke's encephalopathy with ophthalmoplegia, showed similar alterations. 
Erythrocytes were incubated (Warburg) 3 hours at $38^{\circ} \mathrm{C}$ under air with methylene blue and glucose labelled $\left(\mathrm{C}^{14}\right)$ on carbon 1 or 2 (separate flasks). Recoveries of $\mathrm{C}^{14} \mathrm{O}_{2}$ determined separately for the 2 substrates were expressed as percentages of $\mathrm{C}^{14}$ counts added. Oxygen consumption and pentose accumulation were determined.

Erythrocytes from 25 controls (normals and hospital convalescents) showed mean carbon-1 recoveries of $35.6 \pm$ $4.1 \%$, and carbon- 2 of $16.7 \pm 2.2 \%$. Mean oxygen consumption was $108.0 \pm 11 \mu \mathrm{l}$. and pentose accumulation $131 \pm 13 \mu \mathrm{g}$. per flask. Erythrocytes of 8 Wernicke patients pre-treatment showed mean carbon-1 recovery of $36.7 \pm 3.6 \%$ and carbon- 2 of $10.2 \pm 2.1 \%$. Mean oxygen consumption was reduced slightly $(98.4 \mu \mathrm{l}$.) and pentose accumulation increased (169 $\mu \mathrm{g}$. per flask). In vitro addition of thiamin or cocarboxylase produced increased carbon-2 recoveries. After thiamin treatment, with remission of ophthalmoplegia, the patients' erythrocytes showed $31.7 \pm 4.3 \%$ carbon- 1 recovery, $13.8 \pm 2.1 \%$ carbon-2, $105.5 \mu \mathrm{l}$. oxygen consumption and $148 \mu \mathrm{g}$. pentose accumulation.

Significantly reduced carbon-2 recovery $(\mathrm{p}<0.01)$ without change in carbon-1 recovery associated with increased pentose accumulation indicates interference' with glucose oxidation at the transketolase step after removal of the first carbon as $\mathrm{CO}_{3}$. This reduced hexose recycling results from insufficient thiamin as cotransketolase.

\section{Reduction of Reactive Hyperemia Blood Flow of the Foot} by Tobacco Smoking. J. EDWIN Wood and JAY D. Coffman, Boston, Mass. (Introduced by Robert W. Wilkins).

In patients with and without vascular disease foot blood flows were measured with a constant temperature $\left(89^{\circ} \mathrm{F}\right)$ water plethysmograph at 15 -second intervals for two minutes following five minutes of arterial occlusion of the foot. The subject then smoked two cigarettes at his usual rate. Five minutes after smoking began, arterial occlusion was reapplied and the measurements were repeated. By suitable calculation the total volume of blood that flowed through the foot during the two minutes of reactive hyperemia while smoking was compared to that without smoking.

Of 19 subjects studied 20 times in a warm $\left(83^{\circ} \mathrm{F}\right)$ environment, 13 showed a definite decrease in reactive hyperemia blood flow (RHBF) during smoking. Control (warm) RHBF averaged 15.3, ranging from 6.3 to 41.2 cc. per $100 \mathrm{cc}$. of foot. During smoking RHBF averaged 10.1, ranging from 3.0 to $36.2 \mathrm{cc}$. per $100 \mathrm{cc}$. Seven subjects showed no change in RHBF while smoking.

Eight subjects were restudied in a cool $\left(68^{\circ} \mathrm{F}\right)$ environment which produced a definite decrease of "Control" RHBF. Cool control RHBF averaged 10.2, ranging from 5.5 to $16.6 \mathrm{cc}$. per $100 \mathrm{cc}$, and during smoking RHBF decreased in six of the eight and averaged 5.0, ranging from 1.3 to $10.5 \mathrm{cc}$. per $100 \mathrm{cc}$.

RHBF was measured simultaneously in both feet of three patients with unilateral sympathectomy in the warm and cool environment. In five of six studies (three warm, three cool), unsympathectomized limbs showed definite reductions of RHBF during smoking while the sixth showed no change. The contralateral (sympathectomized) limbs showed no change of RHBF in four instances and a diminution of RHBF definitely less than that of the sympathectomized side in two instances.

These studies show that tobacco smoking often reduces post-ischemia vasodilatation, and that this reduction is apparently mediated via the sympathetic nervous system.

\section{Use of Indicator-Dilution Curves in the Diagnosis of Valvular Insufficiency. EDWARD WOODWARD, JR., F. Henry Ellis, JR., and Earl H. Wood,* Rochester, Minn.}

Following rapid injection of an indicator into the vascular system, traversal of the resultant dye-blood mixture through a cardiac chamber, which is guarded by an incompetent valve or at which a left-to-right shunt occurs, results in a decrease in peak concentration, a prolongation of the disappearance slope, and a relative decrease in the appearance time of the indicator-dilution curves recorded down stream to the site of the abnormality. These parameters of the dilution curve also are affected by the cardiac output and central blood volume; hence, their use for detection of valvular insufficiency or a shunt requires means for compensating for variations in flow and volume in the individual patient. The DT/BT ratio, and the variance and disappearance of slope ratios of Korner and Shillingford have been used. The use of these and other parameters of dilution curves in distinguishing between patients with predominant stenosis or predominant insufficiency of the mitral valve has been investigated by a study of dilution curves recorded continuously at the radial artery after sudden single injections of $T-1824$ ( 7.5 to $15 \mathrm{mg}$.) into the pulmonary artery of 11 healthy persons and 30 patients with mitral valvular disease. Further, the relationship of mitral insufficiency to the quantity of dye rapidly appearing in the left atrium following left ventricular injection has been studied in 10 dogs before and after surgical production of insufficiency and in 20 patients with valvular disease.

A correlation was established between the degree of insufficiency and each of 12 parameters studied. None provided a completely uniform separation of patients with predominant stenosis and those with predominant insufficiency. However, frequencies of overlap as low as one in 30 establish the indicator-dilution method as a valuable adjunct to the differential diagnosis of mitral valvular disease.

\section{The Elaboration by Tumor Cells of Lactic Dehydrogenase} into the Bathing Fluids: In Vitro and In Vivo Studies. Felix Wróblewski and Alice E. Moore, New York, N. Y. (Introduced by Rulon W. Rawson).

It has been suggested that cancer cells originate from normal cells in two phases: (1) irreversible injury of respiration and (2) replacement of lost respiration energy by fermentation energy. This hypothesis is consistent 
with the observations that some experimentally induced and clinical states of malignant neoplasia manifest increased serum lactic dehydrogenase activity.

In vitro tissue cultures of normal cells including amnion and fibroblast in Eagles medium were incubated at $37^{\circ} \mathrm{C}$ for ten days; similarly, tissue cultures of malignant neoplastic cells including Osgood J-111 leukemia, Osgood $\mathrm{J}-96$ leukemia, HEP-2, HEP-1, and $\mathrm{HeLa}$ were incubated. The bathing fluid medium of each of the normal and cancerous tissue cultures was examined daily for lactic dehydrogenase activity. The enzyme activity of the media of normal cells remained the same or increased slightly during a ten-day period. The lactic dehydrogenase activity of the media of the cancerous cells increased progressively and reached values of as much as fifteen times the initial enzyme activity of the media.

Pleural and peritoneal effusions from patients with congestive heart failure, cirrhosis, tuberculosis and metastatic cancer were examined for lactic dehydrogenase activity. Simultaneously, the enzyme activity of the serum of the same individuals was determined. In most instances we have found that effusions containing malignant cells manifest lactic dehydrogenase activity greater than the enzyme activity of the serum obtained simultaneously from the same individual. In pleural and peritoneal fluids free of malignant cells we have found lactic dehydrogenase activities which were less than the respective serum lactic dehydrogenase.

It would appear that lactic dehydrogenase is elaborated into the bathing fluids by tumor cells. This characteristic of malignant cells in vitro and in vivo may serve to distinguish biochemically normal from cancerous cells.

Overproduction of Uric Acid as the Cause of Hyperuricemia in Primary Gout. James B. Wynganden, Durham, N. C. (Introduced by Grace P. Kerby).

Stetten and associates demonstrated that certain gouty subjects incorporated excessive quantities of glycine- $\mathrm{N}^{16}$ into urinary urate, whereas certain others did not. These studies have been repeated, employing a tracer dose of glycine-1- $\mathrm{C}^{14}$ in place of glycine- $\mathrm{N}^{15}$. Six consecutive gouty subjects have shown abnormally high concentrations and abnormally great cumulative incorporations of $\mathrm{C}^{14}$ in urinary urate. Five of these subjects had normal urinary urate excretions; in one there was asymptomatic hyperuricemia. Degradation studies of urinary urate have shown that glycine-1- $\mathrm{C}^{14}$ has labeled urate specifically (97 per cent) in C-4. These studies demonstrate that overproduction of urate is the metabolic defect of primary gout. Studies of the labeling patterns of urinary hypoxanthine, xanthine, adenine, guanine and 7-methylguanine suggest that synthesis of urate in control, gouty and leukemic subjects involves two types of pathway, the one an early cleavage of newly formed nucleotides, the other the catabolism of nucleic acids. The data suggest that the first type of pathway participates in the excessive synthesis of labeled urate in primary gout, the second in secondary gout.
These studies permit formulation of a working hypothesis regarding the metabolic defect of primary gout: Normally the quantity of purine nucleotides synthesized somewhat exceeds that required for nucleic acid synthesis. Excess nucleotides are cleaved, and the purine moieties oxidized to urate. The rate of purine nucleotide synthesis is regulated by a feedback system (as recently demonstrated in E. coli for pyrimidine synthesis). In primary gout this feedback mechanism fails to operate effectively. This defect permits excessive production of purine nucleotides, which are promptly cleaved and oxidized, resulting in overproduction of urate by a direct pathway not involving nucleic acids as intermediates. A lesion of the feedback mechanism itself, or an excess activity of the enzymes of nucleotide cleavage, or of base oxidation, is a possible explanation. This hypothesis is consistent with existing information on purine metabolism in normal and gouty persons.

\section{Increased Permeability of Muscle Membranes Produced by Insulin. Kenneth L. ZIERLER,* Baltimore, Md.}

There are two major theories on the mechanism of insulin action, one holding that it acts at an enzymic level, the other that it accelerates movement of glucose across cell membranes. The latter hypothesis may imply that insulin acts on the membrane itself, altering permeability.

When intact rat skeletal muscle is incubated intracellular material as large as proteins diffuses from tissue to solution. Aldolase is one such substance and rate of diffusion of aldolase may be used as an index of muscle membrane permeability.

In 17 pairs of muscles in Krebs-Ringer- $\mathrm{HCO}_{z}$-glucose, aldolase diffusion was increased by 40 per cent by addition of insulin. When the membrane was depolarized by a bathing solution rich in potassium, aldolase diffusion was accelerated greatly. In 30 pairs of muscles in this solution insulin increased aldolase diffusion by an additional 20 per cent, a value significant at $P=0.001$. It is concluded that in isolated muscle under these conditions insulin increased membrane permeability.

Effect of Naphthalene Derivatives on Glutathione Metab-
olism of Erythrocytes from Patients with Naphthalene
Hemolytic Anemia. WILliAM H. ZinKHAM and BAR-
ToN CHILD, Baltimore, Md. (Introduced by C. Lock-
ard Conley).

Hemolytic anemias due to primaquine and certain other drugs have been shown to occur in people with an intrinsic abnormality of the erythrocyte. This abnormality is characterized by relative deficiency of reduced glutathione (GSH) and marked decrease of GSH when erythrocytes are incubated with acetylphenylhydrazine (APH). These erythrocytes are not susceptible to acceleration by naphthalene of taurocholate hemolysis.

Severe hemolytic anemia has occasionally been observed in people exposed to naphthalene $\left(\mathrm{C}_{10} \mathrm{H}_{8}\right)$. To determine the nature of this process, GSH levels before 
and after incubation with $\mathrm{APH}$, naphthalene, and naphthalene metabolites were measured in erythrocytes from 4 negro patients who developed fulminating hemolytic anemia following exposure to naphthalene moth balls. There was marked reduction in GSH after incubation with APH. No significant decrease of GSH was observed when erythrocytes were incubated with naphthalene. Four naphthalene derivatives: alpha- and betanaphthol and alpha- and beta-naphthoquinone, produced a marked fall in GSH. This effect was observed in concentrations which failed to reduce GSH of normal erythrocytes. Menadione sodium bisulfite (Hykinone ${ }^{(8)}$ ), a derivative of alpha-naphthoquinone, was equally effec- tive in decreasing GSH. These experiments indicate that naphthalene metabolites rather than naphthalene are the active hemolytic agents.

This abnormality in GSH metabolism was also observed in a number of relatives of these patients. The distribution of the abnormality within these families demonstrates that this characteristic is genetically determined, and is probably due to a dominant mutant gene. It is noteworthy that in the cases studied, as well as bulk of cases reported in the literature, the patients are negroes. These observations suggest that susceptibility to naphthalene hemolytic anemia may be restricted to certain members of the population. 